\title{
Eisenstein Series, Eta Quotients and Their Applications in Number Theory
}

\author{
by \\ Zafer Selcuk Aygin \\ B.Math., M.Sc. (Bilkent University) \\ A thesis submitted to \\ the Faculty of Graduate and Postdoctoral Affairs \\ in partial fulfillment of \\ the requirements for the degree of
}

\section{Doctor of Philosophy}

in

School of Mathematics and Statistics

Ottawa-Carleton Institute for Mathematics and Statistics

Carleton University

Ottawa, Ontario, Canada

(C) Copyright

2014-2016, Zafer Selcuk Aygin 


\section{Abstract}

We use tools from the theory of modular forms and the interplay between Eisenstein series and eta quotients to deal with some number theoretic curiosities. We describe three of them below.

Let $k \geq 2$ be an integer and $j$ an integer satisfying $1 \leq j \leq 4 k-5$. We define a family $\left\{C_{j, k}(z)\right\}_{1 \leq j \leq 4 k-5}$ of eta quotients, and prove that this family constitute a basis for the space $S_{2 k}\left(\Gamma_{0}(12)\right)$. We then use this basis together with certain properties of modular forms at their cusps to prove an extension of the Ramanujan-Mordell formula.

We express the newforms in $S_{2}\left(\Gamma_{0}(N)\right)$ for various $N$ as linear combinations of Eisenstein series and eta quotients, and list their corresponding strong Weil curves. We use modularity theorem to give generating functions for the order of $E\left(\mathbb{Z}_{p}\right)$ for these strong Weil curves. We then use our generating functions to deduce congruence relations for the order of $E\left(\mathbb{Z}_{p}\right)$.

We determine all the eta quotients in $M_{2}\left(\Gamma_{0}(N)\right)$ for $N \leq 100$. We then determine the Fourier coefficients of four classes of those eta quotients. For example we show that

$$
\frac{\eta^{5}(5 z)}{\eta(z)}=\sum_{n=1}^{\infty}\left(\sum_{d \mid n}\left(\frac{5}{n / d}\right) d\right) e^{2 \pi i n z}
$$

which is due to Ramanujan. We use this equation to give a proof of Ramanujan's partition congruence modulo 5 . 


\section{Acknowledgements}

I have had a very fulfilling experience during my Ph.D. studies, both academically and personally. I would like to take this opportunity to thank the people who have contributed so much to this experience.

I want to start by expressing my gratitude to the Turkish Ministry of Education for their generous financial support throughout my Ph.D. studies.

I would also like to thank my supervisors, Dr. Ayşe Alaca and Dr. Şaban Alaca, for their academic guidance through the studies.

Besides my supervisors, I would like to thank Dr. Kenneth S. Williams whose research has been a continuous inspiration for my studies. I am grateful to Dr. Shaun Cooper who, in our discussion during CNTA XIII, proposed to work on the extension of Ramanujan-Mordell formula proven in this thesis.

I am also grateful to my dear friend, Dr. Fatih Deniz, for his encouragement and wise advices towards achieving my academic goals.

My friends Mustafa Caglayan, David Charles, Wael Elsin, Luzia Jaeger Hintze, Rohat Kaya, Gharam Mahfouz and Aleksandr Tuxanidy made invaluable contributions to my experience in the beautiful city of Ottawa.

Most importantly, I would like to thank my dad, Musa Aygin, and mom, Emine Aygin, for their continuing spiritual support during my academic career. 


\section{Contents}

Abstract .................................. ii

Acknowledgements ................... . iii

Symbol Index . . . . . . . . . . . . . . . . . . viii

\begin{tabular}{lll}
\hline 1 & Introduction & 1
\end{tabular}

\begin{tabular}{lll}
\hline I Preliminaries & 13
\end{tabular}

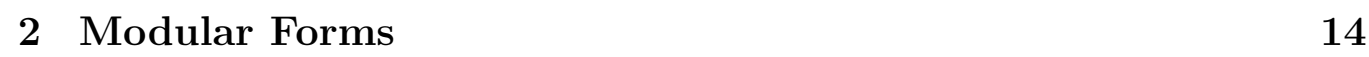

2.1 Dirichlet Characters . . . . . . . . . . . . . . . 15

2.2 Modular Subgroup $\Gamma_{0}(N)$. . . . . . . . . . . . . . . . . . 17

2.3 Modular Forms . . . . . . . . . . . . . . . . . . . . . . 20

2.4 Some Properties of Modular Forms . . . . . . . . . . . . . . . 26

\begin{tabular}{lll}
\hline 3 & Eisenstein Series & 27
\end{tabular}

3.1 Bernoulli Numbers . . . . . . . . . . . . . . . . . . . . . . . . . . 28

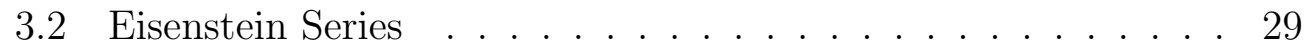


$3.3 \quad$ Bases of $E_{k}\left(\Gamma_{0}(N)\right)$ for a Family of $N$ 's . . . . . . . . . . . 32

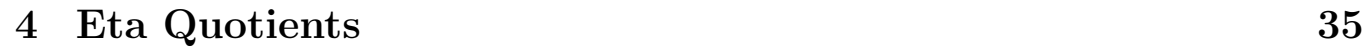

4.1 Dedekind Eta Function . . . . . . . . . . . . . . . . . . . . . . 36

4.2 Eta Quotients . . . . . . . . . . . . . . . . . . 38

$4.3 \quad$ Eta Quotients in $M_{2}\left(\Gamma_{0}(16), \chi_{8}\right) \ldots \ldots$. . . . . . . . . . 46

$4.4 \quad$ Eta Quotients in $M_{2}\left(\Gamma_{0}(N)\right)$ for $N=p_{1} p_{2} \ldots \ldots$. . . . . 49

$4.5 \quad$ Number of Eta Quotients in $M_{2}\left(\Gamma_{0}(N)\right)$ for $N \leq 100$. . . . 57

II Main Research $\quad 60$

5 Bases for $S_{k}\left(\Gamma_{0}(12), \chi\right)$ for $\chi=\chi_{1}, \chi_{-3}, \chi_{-4}, \chi_{12}$

$5.1 \quad$ New Families of Eta Quotients and Bases for $S_{k}\left(\Gamma_{0}(12), \chi\right)$. . 62

6 Fourier Series Expansions of Some Modular Forms at Certain

$\begin{array}{lc}\text { Cusps } & 66\end{array}$

$6.1 \quad$ Fourier Series Expansions of $\varphi(z)$ and $\varphi(3 z)$ at Certain Cusps 66

6.2 Fourier Series Expansions of $E_{2 k}(t z)$ at Certain Cusps . . . . . 72

\begin{tabular}{|lll|}
\hline 7 & On an Extension of the Ramanujan-Mordell Theorem & 74
\end{tabular}

$7.1 \quad$ An Extension of Ramanujan-Mordell Theorem . . . . . . . . . 75

7.2 Examples and Remarks . . . . . . . . . . . . . . . . . . . 79

8 Generating Functions for Number of Algebraic Points on an $\begin{array}{ll}\text { Elliptic Curve on Prime Characteristic Fields } & 83\end{array}$ 
8.1 Newforms in Terms of Eisenstein Series and Eta Quotients . . 84

8.2 Some Arithmetic Properties of $\left|E\left(\mathbb{Z}_{p}\right)\right|$ for Certain Elliptic Curves . . . . . . . . . . . . . . . . . . 90

\section{Further Results and Future Research $\quad 96$}

$9 \quad$ Bases for $M_{3}\left(\Gamma_{0}(24), \chi\right)$ and $M_{2}\left(\Gamma_{0}(108), \chi\right) \quad 97$

$9.1 \quad$ Bases for $M_{3}\left(\Gamma_{0}(24), \chi\right)$. . . . . . . . . . . . . . . . . . . . . . 98

$9.2 \quad$ Bases for $M_{2}\left(\Gamma_{0}(108), \chi\right) \ldots \ldots$. . . . . . . . . . 102

10 Representations by Certain Quadratic Forms 105

10.1 Sextenary Quadratic Forms . . . . . . . . . . . . 106

10.2 Quaternary Quadratic Forms . . . . . . . . . . . . . 119

11 Fourier Coefficients of Certain Families of Eta Quotients 126

11.1 Fourier Coefficients of Eta Quotients . . . . . . . . . . . . . . 127

11.2 A Proof for Ramanujan's Congruence for $p(n)$ Modulo 5 . . . 136

11.3 Arithmetic Properties of Fourier Coefficients of Eta Quotients in $M_{2}\left(\Gamma_{0}(10), \chi_{5}\right)$. . . . . . . . . . . . . . . . . . . 138

11.4 Number of Eta Quotients in $E_{2}\left(\Gamma_{0}(N)\right)$ for $N<100$. . . . . . 140

12 Future Research and Conclusion 142

12.1 Bases for $S_{k}\left(\Gamma_{0}(N), \chi\right)$ in Terms of Eta Quotients . . . . . . . 142

12.2 Extensions of Ramanujan-Mordell Formula . . . . . . . . . . . 143

12.3 Half-Integral Weight Modular Forms . . . . . . . . . . . . . . 147 
12.4 Application to Convolution Sums . . . . . . . . . . . . . . . 150

12.5 Further Discussions . . . . . . . . . . . . . . . . . . . . 151

12.5.1 A Set of Criteria for an Eta Quotient to be in $E_{k}\left(\Gamma_{0}(N), \chi\right) 152$

12.5.2 On Weight 2 Newforms and Elliptic Curves . . . . . . 152

12.5.3 On Larger Weight Newforms . . . . . . . . . . . . . . . 154

\begin{tabular}{ll}
\hline Appendices & 164
\end{tabular}

\begin{tabular}{|ll} 
Appendix A Bases for $S_{2 k}\left(\Gamma_{0}(20)\right)$ and $S_{2 k}\left(\Gamma_{0}(28)\right)$ & 164
\end{tabular}

Appendix B Newforms in $M_{2}\left(\Gamma_{0}(N)\right)$ for various $N<100 \quad 168$

\begin{tabular}{lll}
\hline Appendix C & Bases for $M_{2}\left(\Gamma_{0}(N)\right)$ for various $N<100$ & 171
\end{tabular} 
viii

\section{Symbol Index}

\begin{tabular}{|c|c|c|c|}
\hline Symbol & page & Symbol & page \\
\hline $\mathbb{N}, \mathbb{Z}, \mathbb{Q}, \mathbb{R}, \mathbb{C}, \mathbb{H}, \mathbb{N}_{0}$ & 1 & $S(2 k, N)$ & 26 \\
\hline $\mathbb{Z}_{p}$ & 1 & $B_{k}, B_{k, \chi}$ & 28 \\
\hline$\eta(z)$ & 4 & $\sigma_{(k, \chi, \psi)}(n)$ & 30 \\
\hline$N\left(a_{1}^{l_{1}}, \ldots, a_{k}^{l_{k}} ; n\right)$ & 7 & $\sigma_{k}(n)$ & 30 \\
\hline$\varphi(z)$ & 7 & $E_{k, \chi, \psi}(z)$ & 30 \\
\hline$[k] f(q)$ & 8 & $E_{2 k}(z)$ & 31 \\
\hline$E\left(\mathbb{Z}_{p}\right)$ & 9 & $L_{t}(z)$ & 32 \\
\hline$p(n)$ & 12 & $\eta_{N}\left[r_{\delta_{1}}, \ldots, r_{\delta_{\sigma_{0}(N)}}\right](z)$ & 36 \\
\hline$\left(\frac{d}{n}\right), \chi_{d}(n)$ & 17 & $\eta_{t}(z)$ & 37 \\
\hline$\Gamma_{0}(N)$ & 18 & $p^{s} \| N$ & 39 \\
\hline$\phi(n)$ & 19 & $C_{j, k}(z), D_{j, k}(z)$ & 62 \\
\hline$R\left(\Gamma_{0}(N)\right)$ & 19 & $E_{(2 k, t)}(z)$ & 72 \\
\hline$M_{k}\left(\Gamma_{0}(N), \chi\right)$ & 21 & $S_{k}^{\text {old }}\left(\Gamma_{0}(N), \chi\right)$ & 83 \\
\hline$M_{k}\left(\Gamma_{0}(N)\right)$ & 21 & $S_{k}^{n e w}\left(\Gamma_{0}(N), \chi\right)$ & 83 \\
\hline$v_{r}(f(z))$ & 22,24 & $S(k, N, \chi)$ & 97 \\
\hline$S_{k}\left(\Gamma_{0}(N), \chi\right), S_{k}\left(\Gamma_{0}(N)\right)$ & 25 & $E(k, N, \chi)$ & 97 \\
\hline$E_{k}\left(\Gamma_{0}(N), \chi\right), E_{k}\left(\Gamma_{0}(N)\right)$ & 25 & & \\
\hline
\end{tabular}




\section{Chapter 1}

\section{Introduction}

Let $\mathbb{N}, \mathbb{Z}, \mathbb{Q}, \mathbb{R}, \mathbb{C}, \mathbb{H}$ denote the sets of natural numbers, integers, rational numbers, real numbers, complex numbers and upper half plane of complex numbers, respectively, and we let $\mathbb{N}_{0}=\mathbb{N} \cup\{0\}$. $\mathbb{Z}_{p}$ denotes field of $p$ elements. This thesis consists of three parts. In the first part we introduce modular forms, Eisenstein series and eta quotients. Although it includes some new results, most of the first part is derived from known results. The main and interesting research of the thesis is given in the second part. It includes an important family of eta quotients, series expansions of modular forms at different cusps, an extension of the Ramanujan-Mordell formula and generating functions for the number of algebraic points on certain elliptic curves over $\mathbb{Z}_{p}$, where $p$ is a prime. The third part is where we are having fun with our background on Eisenstein series and eta quotients and apply them to some classical problems such as representation of numbers by certain quadratic 
forms and infinite product to infinite sum formulas. The results of the third part are actually examples of calculations for the research we want to conduct in future. In the last chapter we give an idea of where else the results of this thesis can be applied and how we can stretch this research further.

Modular forms are basically holomorphic complex functions that satisfy certain transformation properties. We give a brief introduction to modular forms in Chapter 2. That chapter is a combination of results from [70] and [40], as well as some observations. Our observations are done by restricting the weight of the modular form to be an integer, the modular subgroup to be $\Gamma_{0}(N)$ and the multiplier system to be a real Dirichlet character. Theorem 2.3.5 gives an insight on the behaviour of the modular forms at cusps which play an important role in the study of modular forms and in particular in this thesis. It is also worth noting that Theorem 2.4.4 (which is known as the Sturm bound) is a strong tool to show that particular modular forms are equal. We will check the equality of given modular forms by just checking the equality of the first few coefficients in their Fourier series representations. In Chapter 8 we use this theorem to write newforms in terms of Eisenstein series and eta quotients.

We write $M_{k}\left(\Gamma_{0}(N), \chi\right)$ to denote the space of modular forms for $\Gamma_{0}(N)$ of weight $k$ with multiplier system $\chi$, and $E_{k}\left(\Gamma_{0}(N), \chi\right)$ and $S_{k}\left(\Gamma_{0}(N), \chi\right)$ to denote the subspaces of Eisenstein forms and cusp forms of $M_{k}\left(\Gamma_{0}(N), \chi\right)$, respectively. When $\chi$ is the primitive principal character we simply write $M_{k}\left(\Gamma_{0}(N)\right)$ for the modular space $M_{k}\left(\Gamma_{0}(N), \chi\right)$, similarly we write $E_{k}\left(\Gamma_{0}(N)\right)$ 
and $S_{k}\left(\Gamma_{0}(N)\right)$ for the Eisenstein subspace and cusp form subspace, respectively. It is known that $M_{k}\left(\Gamma_{0}(N), \chi\right)$ is a linear vector space and that, (see for example [70, p. 83] and [67])

$$
M_{k}\left(\Gamma_{0}(N), \chi\right)=E_{k}\left(\Gamma_{0}(N), \chi\right) \oplus S_{k}\left(\Gamma_{0}(N), \chi\right) .
$$

In this thesis we give bases of $E_{k}\left(\Gamma_{0}(N), \chi\right)$ in terms of Eisenstein series and bases of $S_{k}\left(\Gamma_{0}(N), \chi\right)$ in terms of eta quotients. We always choose eta quotients, in the basis, to be of different vanishing orders at infinity for computational advantage.

Let $k \in \mathbb{N}$ and $\chi$ and $\psi$ be primitive characters. As in [40] throughout the thesis we use the notation $q=e(z)=e^{2 \pi i z}$ with $z \in \mathbb{H}$, and so $|q|<1$. We define the Eisenstein series $E_{k, \chi, \psi}(z)$ by

$$
E_{k, \chi, \psi}(z)=c_{0}+\sum_{n \geq 1}\left(\sum_{d \mid n} \chi(d) \psi(n / d) d^{k-1}\right) q^{n}
$$

where $c_{0}$ is a constant, see (3.2.2). We introduce the Eisenstein series in Chapter 3. Theorem 3.2.1 gives the criteria on $k, \chi$ and $\psi$ which determines if a certain Eisenstein series is a modular form. A basis of $E_{k}\left(\Gamma_{0}(N), \chi\right)$ in terms of Eisenstein series is given in Theorem 3.2.2. These results have been derived from [70] and [51].

The Dedekind eta function $\eta(z)$ is the holomorphic function defined on 
the upper half plane $\mathbb{H}=\{z \in \mathbb{C} \mid \operatorname{Im}(z)>0\}$ by the product formula

$$
\eta(z)=e^{\pi i z / 12} \prod_{n=1}^{\infty}\left(1-e^{2 \pi i n z}\right) .
$$

Let $N \in \mathbb{N}$ and $r_{\delta} \in \mathbb{Z}$ for all $1 \leq \delta \mid N$, where the $r_{\delta}$ are not all zero. Let $z \in \mathbb{H}$. We define an eta quotient by the product formula

$$
f(z)=\prod_{1 \leq \delta \mid N} \eta^{r_{\delta}}(\delta z) .
$$

We start Chapter 4 with an introduction to the Dedekind eta function and eta quotients. Then we give necessary and sufficient conditions for an eta quotient to be in $M_{k}\left(\Gamma_{0}(N), \chi\right)$ in Theorems 4.2.4 and 4.2.6, respectively. In the third section we use Theorems 4.2 .4 and 4.2 .6 to give all the eta quotients in $M_{2}\left(\Gamma_{0}(16), \chi_{8}\right)$. In Section 4.4 , we go further and give lists of eta quotients in $M_{2}\left(\Gamma_{0}(N)\right)$, for all $N$ which are multiple of two distinct primes. As mentioned earlier, we give bases for $S_{k}\left(\Gamma_{0}(N), \chi\right)$ in terms of eta quotients. This is not always possible, but there are many interesting cusp form spaces that can be generated by linear combinations of eta quotients. In Section 4.5, we give the number of eta quotients in $M_{2}\left(\Gamma_{0}(N)\right)$, where $N \leq 100$, in Figures 4.5.1 and 4.5.2.

In the first four chapters we build some basic familiarity with Eisenstein series, eta quotients and their modularity. The rest of the thesis will be applications of the previous observations, findings and calculations. 
If possible it is usually an easy exercise of Theorems 4.2 .4 and 4.2 .6 to find a basis for given $S_{k}\left(\Gamma_{0}(N), \chi\right)$ in terms of eta quotients. You can find many such examples in Appendix C. We were curious to find a family of such spaces. For this purpose we fixed the level at $N=12$. Then by examining the behaviour of various eta quotients in $S_{k}\left(\Gamma_{0}(N), \chi\right)$ at the cusps of $\Gamma_{0}(12)$, we found a family of eta quotients which form a basis for $S_{k}\left(\Gamma_{0}(12), \chi\right)$, for all $k>1$ and for all $\chi$, a real character with conductor dividing 12 . This exploration is one of the most important results in this thesis. This family of eta quotients will be given in Chapter 5. Note that we have chosen this family in such a way that the eta quotients in the family have different vanishing orders at infinity when $k$ is fixed. This idea is similar to the idea of the so-called 'Miller basis' (see, [70, Section 2.3]). This family is important in a couple of aspects. As far as we are aware, it is the first example of a family of eta quotients to generate $S_{k}\left(\Gamma_{0}(N)\right)$, for all $k>1$. Also we use this family of eta quotients, together with the Eisenstein series we give in Example 3.3 .2 and certain properties of modular forms at cusps, to give an extension of the Ramanujan-Mordell formula in Chapter 7. An exciting fact in this line of research is that, $N=12$ is not a unique example. In Appendix A. we give other families of eta quotients which give bases for $S_{2 k}\left(\Gamma_{0}(20)\right)$ and $S_{2 k}\left(\Gamma_{0}(28)\right)$, for all $k>1$. And we are sure that there many such examples, recent findings in [65] support this idea.

Chapter 6 is a relatively short chapter, but technically it is of prime importance. In Section 6.1 we compute Fourier series expansions of Dedekind 
eta functions $\eta(t z)$, for all $t \mid 12$, at relevant cusps of $\Gamma_{0}(12)$. This result is an application of [40, Proposition 2.1], and requires careful choice of matrices and tedious calculations. In Section 6.2, we find Fourier series expansions of the Eisenstein series $E_{2 k}(t z)$, for all $k>1$ and $t \in \mathbb{N}$, at the cusps $\frac{1}{c}$, for all $c \in \mathbb{N}$. This result in Section 6.2 is new. Results of Section 6.1 together with the results of Section 6.2 help us give an extension of the Ramanujan-Mordell formula. It is also worth noting that, these results might be applied to many other problems in modular forms.

Let $k \in \mathbb{N}$ and $a_{i} \in \mathbb{N}(1 \leq i \leq k)$. We fix the notation for number of representations of a non-negative integer $n$, by the quadratic form

$$
a_{1} x_{1}^{2}+a_{2} x_{2}^{2}+\cdots+a_{k} x_{k}^{2}
$$

by

$N\left(a_{1}, a_{2}, \ldots, a_{k} ; n\right)=\left|\left\{\left(x_{1}, x_{2}, \ldots, x_{k}\right) \in \mathbb{Z}^{k} \mid n=a_{1} x_{1}^{2}+a_{2} x_{2}^{2}+\cdots+a_{k} x_{k}^{2}\right\}\right|$.

The order of the $a_{i}$ in 1.0.3 doesn't matter. So for

$$
N\left(a_{1}, \ldots, a_{1}, a_{2}, \ldots, a_{2}, \ldots, a_{k}, \ldots, a_{k} ; n\right)
$$


where $a_{j}$ is repeated $l_{j}$ times $(1 \leq j \leq k)$, we can use the shorthand notation

$$
N\left(a_{1}^{l_{1}}, a_{2}^{l_{2}}, \ldots, a_{k}^{l_{k}} ; n\right)
$$

Following Ramanujan's notation, let us define

$$
\varphi(z)=\sum_{n=-\infty}^{\infty} q^{n^{2}}
$$

Then we have

$$
\sum_{n=0}^{\infty} N\left(a_{1}^{l_{1}}, a_{2}^{l_{2}}, \ldots, a_{k}^{l_{k}} ; n\right) q^{n}=\prod_{i=1}^{k} \varphi^{l_{i}}\left(a_{i} z\right)
$$

On the other hand, by the Jacobi triple product identity [17, Corollary 1.3.4], we have

$$
\varphi(z)=\frac{\eta^{5}(2 z)}{\eta^{2}(z) \eta^{2}(4 z)}
$$

That is we can express the generating function of $N\left(a_{1}^{l_{1}}, a_{2}^{l_{2}}, \ldots, a_{k}^{l_{k}} ; n\right)$ in terms of eta quotients. Letting $\sum_{i=1}^{k} l_{i}$ be an even number, it is well known that the function $\prod_{i=1}^{k} \varphi^{l_{i}}\left(a_{i} z\right)$ is a modular form of integer weight. We determine the level, weight and multiplier system of $\prod_{i=1}^{k} \varphi^{l_{i}}\left(a_{i} z\right)$, using Theorem 4.2.6.

Ramanujan in [62] stated and Mordell in [53] proved a formula for $N\left(1^{2 k} ; n\right)$ for all $k \geq 1$. In Chapter 7 , we prove a formula for $N\left(1^{4 k-2 i}, 3^{2 i} ; n\right)$, for all 
$k>1$ and $0 \leq i \leq 2 k$, as an extension of the Ramanujan-Mordell formula. Using results from Chapter 4 , it is easy to show that

$$
\sum_{n=0}^{\infty} N\left(1^{4 k-2 i}, 3^{2 i} ; n\right) q^{n}=\varphi^{4 k-2 i}(z) \varphi^{2 i}(3 z) \in M_{2 k}\left(\Gamma_{0}(12)\right) .
$$

Since we know the basis for $M_{2 k}\left(\Gamma_{0}(12)\right.$ ) (by Example 3.3 .2 and Theorem 5.1.3, we can give a formula for 1.0.5. And comparing coefficients of $q^{n}$ on both sides of it we can find a formula for $N\left(1^{4 k-2 i}, 3^{2 i} ; n\right)$, for all $k>1$ and $0 \leq i \leq 2 k$. The choice of the basis for $S_{2 k}\left(\Gamma_{0}(12)\right)$ plays an important role in the proof.

Between 1955 and 1957, Shimura, Taniyama and Weil conjectured relationships between modular forms and elliptic curves, which was known as the Shimura-Taniyama-Weil conjecture. In 1995, Wiles proved the modularity of semistable elliptic curves, which implied Fermat's Last Theorem, see [71]. Later in 2001, the Shimura-Taniyama-Weil conjecture was proven and is now referred to as modularity theorem, see [18]. In this thesis we will use the $a_{p}$ version of the modularity theorem as given in [28, Theorem 8.8.1], which gives a correspondence between the number of algebraic points on an elliptic curve over $\mathbb{Z}_{p}$ and the $p^{\text {th }}$ coefficient of a newform's Fourier series expansion at infinity, where $p$ is a prime number. Note that we write $[j] f(z)=a_{j}$ for $f(z)=\sum_{n=0}^{\infty} a_{n} q^{n}$.

Theorem 1.0.1. (Modularity Theorem, Version $a_{p}$ ) Let $E$ be an elliptic 
curve over $\mathbb{Q}$ with conductor $N$ given by

$$
y^{2}+a_{1} x y+a_{3} y=x^{3}+a_{2} x^{2}+a_{4} x+a_{6},
$$

where $a_{1}, a_{2}, a_{3}, a_{4}, a_{6} \in \mathbb{Z}$. Let

$E\left(\mathbb{Z}_{p}\right)=\{\infty\} \cup\left\{(x, y) \in \mathbb{Z}_{p} \times \mathbb{Z}_{p} \mid y^{2}+a_{1} x y+a_{3} y=x^{3}+a_{2} x^{2}+a_{4} x+a_{6}\right\}$.

Then for some newform $f_{E} \in S_{2}\left(\Gamma_{0}(N)\right)$ we have

$$
\left|E\left(\mathbb{Z}_{p}\right)\right|=p+1-[p] f_{E}(z) \text { for } p \nmid N .
$$

In [49], Martin and Ono listed all weight 2 newforms that are eta quotients and gave their corresponding strong Weil curves. Such eta quotients exist only for levels 11, 14, 15, 20, 24, 27, 32, 36, 48, 64, 80, 144.

In Section 8.1 we express newforms in $S_{2}\left(\Gamma_{0}(N)\right)$ for $N=11,14,15$, $20,24,27,30,32,33,34,40,42,44,45,56,64$ as linear combinations of Eisenstein series and eta quotients. The coefficients of Fourier expansions of newforms in levels less than 1000 are given in [27]. We use these coefficients and the Sturm bound to prove our results in Section 8.1. In Section 8.2, we use [27, Table 1] to give strong Weil curves corresponding to newforms given in Section 8.1. Then the mapping in Theorem 1.0.1 gives generating functions for $\left|E\left(\mathbb{Z}_{p}\right)\right|$ in terms of Eisenstein series and eta quotients. Then we use these generating functions to give some basic arithmetic properties of 
$\left|E\left(\mathbb{Z}_{p}\right)\right|$. Note that these generating functions are not unique. We choose the generating function which gives us more clues about the arithmetic properties of $\left|E\left(\mathbb{Z}_{p}\right)\right|$. This completes second part of the thesis.

In the third part of the thesis we will work on other applications of Eisenstein series and eta quotients. We will also present a portion of the foundation we set up for our further research.

In Chapter 9 we give explicit bases for some modular form spaces. In Section 9.1, we use the methods of this thesis to give a basis for $M_{3}\left(\Gamma_{0}(24), \chi\right)$, where $\chi$ is a real character with conductor dividing 24 .

As mentioned above, in Chapter 5 we find a basis for $S_{2 k}\left(\Gamma_{0}(12)\right)$, for all $k>1$. The next natural question would be to find a basis for $S_{2}\left(\Gamma_{0}\left(4 \cdot 3^{i}\right)\right)$ for all $i \geq 1$ in terms of eta quotients. Consider the following theorem from 65 .

Theorem 1.0.2. ([65, Theorem 2]) Let $N \in \mathbb{N}, r_{\delta} \in \mathbb{Z}$ for all $\delta \mid N$ (not all zeros) and $p$ be a prime number. Let $f(z)$ be an eta quotient given by

$$
f(z)=\prod_{1 \leq \delta \mid N} \eta^{r_{\delta}}(\delta z)
$$

Then if $f(z) \in M_{k}\left(\Gamma_{0}(N)\right)$, we have

$$
\sum_{d \mid N}\left|r_{\delta}\right| \leq 2 k \prod_{p \mid N}\left(\frac{p+1}{p-1}\right)^{\min \left\{2, \operatorname{ord}_{p}(N)\right\}} .
$$

That is fixing the weight, the number of eta quotients in a modular form 
space is finite as level $N$ goes to infinity, but the dimension tends to infinity as level $N$ goes to infinity. That is, we can't give a set of eta quotients which could generate $S_{2}\left(\Gamma_{0}\left(4 \cdot 3^{i}\right)\right)$ for all $i \geq 1$.

In Section 9.2, we give a set of eta quotients which form a basis for the space $S_{2}\left(\Gamma_{0}\left(4 \cdot 3^{3}\right), \chi\right)$ for all $\chi$, where $\chi$ is a real character with conductor dividing $4 \cdot 3^{3}$.

Note that it is possible to generate many other modular form spaces with Eisenstein series and eta quotients. We give a list of such spaces along with the bases in Appendix $\mathrm{C}$.

In Chapter 10, we use those bases obtained in Chapter 9, to give formulas for $N\left(1^{l_{0}}, 3^{l_{1}}, 9^{l_{2}}, 27^{l_{3}} ; n\right)$ for all $l_{0}, l_{1}, l_{2}, l_{3} \in \mathbb{N}_{0}$, with $l_{0}+l_{1}+l_{2}+l_{3}=4$ and $N\left(1^{l_{1}}, 2^{l_{2}}, 3^{l_{3}}, 6^{l_{6}} ; n\right)$ for all $l_{1}, l_{2}, l_{3}, l_{6} \in \mathbb{N}_{0}$, with $l_{1}+l_{2}+l_{3}+l_{6}=6$. Results of this chapter are new. Using bases from Appendix C, it is possible to give many examples of such representations. Interested readers are encouraged to work on those spaces to find similar formulas.

Fourier coefficients of eta quotients is an interesting study, for contemporary results on this see [1, 16, 21, 25, 26, 32, 41, 54, 72]. In [4, 8, 15, 9, 10], we added many new results to the literature. In Chapter 11, we work on the relations between Eisenstein series and eta quotients to give Fourier coefficients of 4 families of eta quotients. When an eta quotient is in $E_{k}\left(\Gamma_{0}(N), \chi\right)$, by Theorem 3.2 .2 it is possible to write that eta quotient as a linear combination of Eisenstein series, from which we obtain Fourier coefficients of the eta quotient explicitly. In Chapter 11, we give such eta quotients from 
$M_{2}\left(\Gamma_{0}(8), \chi_{1}\right), M_{2}\left(\Gamma_{0}(8), \chi_{8}\right), M_{2}\left(\Gamma_{0}(9), \chi_{1}\right), M_{2}\left(\Gamma_{0}(10), \chi_{5}\right)$. Among those a particularly beautiful example is

$$
\frac{\eta^{5}(5 z)}{\eta(z)}=\sum_{n=1}^{\infty}\left(\sum_{d \mid n}\left(\frac{5}{n / d}\right) d\right) q^{n} \in M_{2}\left(\Gamma_{0}(10), \chi_{5}\right) .
$$

This equation is due to Ramanujan, see [11, 64]. Using this equation we can give a proof of Ramanujan's congruence relation for the partition function $p(n)$, see [17, Theorem 4.4.1], 63].

$$
p(5 n+4) \equiv 0 \quad(\bmod 5)
$$

In Section 11.3, we give some arithmetic relations between Fourier coefficients of eta quotients from Section 11.1. In Figure 11.4.1 we give the numbers of eta quotients in $E_{2}\left(\Gamma_{0}(N)\right)$ for all $N<100$. Examining these eta quotients we have spotted a lot of similarities. These findings suggest a study to see if there is a set of criteria that determine if an eta quotient is in $E_{k}\left(\Gamma_{0}(N)\right)$. Actually this will be the first part of a line of research to come in the next several years.

In Chapter 12, we discuss some future research. 
Part I

\section{Preliminaries}




\section{Chapter 2}

\section{Modular Forms}

In this chapter we give a brief introduction to modular forms. This chapter mostly consists of well known results and some observations that we will use in later chapters. In this thesis, we work on modular forms whose multiplier systems are real Dirichlet characters. The first section introduces the Dirichlet characters. We define the Kronecker symbol, which represents all real characters (see Theorem 2.1.7). Throughout the thesis all multiplier systems will be given in terms of the Kronecker symbol. In the second section, we define the modular subgroup $\Gamma_{0}(N)$ and introduce the 'cusps' of the modular subgroup. In the third section, we give the definition of modular forms and explain the behaviour of the modular forms at cusps. Then we state some of our observations on integer weight modular forms for $\Gamma_{0}(N)$ with real character multiplier system. The last section consists of basic observations on modular forms and we state a theorem regarding the Sturm bound. 


\subsection{Dirichlet Characters}

This section follows [70, Chapter 4] and [52, Section 9.3]. Dirichlet characters play an important role in the theory of modular forms. In this thesis we will use Dirichlet characters to represent multiplier systems for modular forms, and to give Eisenstein series in modular form spaces. We start with the most general definition of the Dirichlet character.

Definition 2.1.1. [70, Definition 4.1] Let $R$ be an integral domain and $\zeta$ be a root of unity. A Dirichlet character (or simply character) of modulus $N$ over $R$ is a map $\varepsilon: \mathbb{Z} \rightarrow R$ such that there is a homomorphism

$$
f:(\mathbb{Z} / N \mathbb{Z})^{*} \rightarrow<\zeta>
$$

for which

$$
\varepsilon(a)= \begin{cases}0 & \text { if } \operatorname{gcd}(a, N)>1 \\ f(a \quad(\bmod N)) & \text { if } \operatorname{gcd}(a, N)=1\end{cases}
$$

The group of such Dirichlet characters (or simply characters) is denoted by $D(N, R)$. We define the conductor of a character and primitive characters as follows.

Definition 2.1.2. [70, Definition 4.18] The conductor of a character $\varepsilon \in$ $D(N, R)$ is the smallest positive divisor $c \mid N$, such that there is a character $\varepsilon^{\prime} \in D(c, R)$ for which $\varepsilon(a)=\varepsilon^{\prime}(a)$ for all $a \in \mathbb{Z}$ with $\operatorname{gcd}(a, N)=1$. A 
character is primitive if its modulus equals its conductor. The character $\varepsilon^{\prime}$ associated to $\varepsilon$ with modulus equal to the conductor of $\varepsilon$ is called the primitive character associated to $\varepsilon$.

Definition 2.1.3. [52, p. 295] A character $\varepsilon \in D(N, \mathbb{Z})$ is real if all its values are real, that is $\varepsilon(n)=-1,0$ or 1 . And a character $\varepsilon \in D(N, \mathbb{Z})$ is a quadratic character if it has order 2 in $D(N, \mathbb{Z})$.

Note that in this thesis, unless otherwise stated all characters considered are real characters.

For example, the Legendre symbol $\left(\frac{n}{p}\right)$ is a primitive real character modulo $p$, where $p$ is a prime number. Legendre symbols are limited when it comes to representing real characters; we extend the Legendre symbol to the Kronecker symbol to represent further real characters.

Definition 2.1.4. [52, $p$. 296] We say $d$ is a quadratic discriminant if, either

$$
\begin{aligned}
& d \equiv 1 \quad(\bmod 4) \text { and } d \text { is squarefree, or } \\
& 4 \mid d, d / 4 \equiv 2 \text { or } 3 \quad(\bmod 4) \text { and } d / 4 \text { is squarefree. }
\end{aligned}
$$

Definition 2.1.5. [52, p. 296] For each quadratic discriminant $d$ we define the Kronecker symbol $\left(\frac{d}{n}\right)$ as follows.

$(i) \quad\left(\frac{d}{2}\right)=\left\{\begin{array}{rlr}0 & \text { when } d \equiv 0 & (\bmod 4) \\ 1 & \text { when } d \equiv 1 & (\bmod 8) \\ -1 & \text { when } d \equiv 5 & (\bmod 8)\end{array}\right.$ 
(ii) Let $p>2$, be a prime, then

$$
\left(\frac{d}{p}\right)=\left\{\begin{aligned}
0 & \text { when } p \mid d \\
1 & \text { when } d \text { is a quadratic residue modulo } p \\
-1 & \text { when } d \text { is a quadratic non-residue modulo } p
\end{aligned}\right.
$$

(iii) $\left(\frac{d}{-1}\right)=\left\{\begin{array}{rc}1 & \text { when } d>0, \\ -1 & \text { when } d<0 .\end{array}\right.$

(iv) $\quad\left(\frac{d}{n}\right)$ is a totally multiplicative function on $n$.

Notation 2.1.6. In this thesis, for $d$ a quadratic discriminant, we use $\chi_{d}(n)$ to denote the Kronecker symbol $\left(\frac{d}{n}\right)$. The primitive principal character is denoted by $\chi_{1}(n)$ (i.e. $\chi_{1}(n)=1$ for all $n \in \mathbb{Z}$ ).

Theorem 2.1.7. ([52, Theorem 9.13]) Let $d$ be a quadratic discriminant. Then $\chi_{d}(n)$ is a primitive quadratic character modulo $|d|$, and every primitive quadratic character is given uniquely in this way.

Let $d$ be a quadratic character, by Theorem 2.1.7, we have $\chi_{d}(n)$ is a character modulo $|d|$. Furthermore, since any real character is either a quadratic character or the primitive principal character, the theorem implies that all real characters can be represented by $\chi_{d}$.

\subsection{Modular Subgroup $\Gamma_{0}(N)$}

This section follows the arguments and definitions from [70, Section 1.3]. 
Definition 2.2.1. [70, Definition 1.9] A congruence subgroup $\Gamma$ of $S L_{2}(\mathbb{Z})$ is any subgroup of $S L_{2}(\mathbb{Z})$ that contains

$$
\Gamma(N)=\operatorname{Ker}\left(S L_{2}(\mathbb{Z}) \rightarrow S L_{2}(\mathbb{Z} / N \mathbb{Z})\right)
$$

for some positive integer $N$. The smallest such $N$ is the level of $\Gamma$.

$$
\begin{gathered}
\text { An element } M=\left(\begin{array}{ll}
a & b \\
c & d
\end{array}\right) \in S L_{2}(\mathbb{Z}) \text { acts on } \mathbb{H} \cup \mathbb{Q} \cup\{\infty\} \text { by } \\
M(z)= \begin{cases}\frac{a z+b}{c z+d} & \text { if } z \neq \infty, \\
\frac{a}{c} & \text { if } z=\infty .\end{cases}
\end{gathered}
$$

Let $z_{1}, z_{2} \in \mathbb{H} \cup \mathbb{Q} \cup\{\infty\}$. If there exists an $M \in \Gamma$, such that $M\left(z_{1}\right)=z_{2}$, then we say $z_{1}$ and $z_{2}$ are equivalent for $\Gamma$. It is clear that this action is an equivalence relation on $\mathbb{H}$. The set formed by representatives from each equivalence class in $\mathbb{H}$ is called the fundamental domain, see [39, Section 3.1], [34, Section 1.5], [28, Section 2.3], [44, Section 1.1].

The elements of $\mathbb{Q} \cup\{\infty\}$ are called cusps. It is also clear that this action is an equivalence relation on $\mathbb{Q} \cup\{\infty\}$. We choose a representative from each equivalence class in $\mathbb{Q} \cup\{\infty\}$ and form a set $R(\Gamma)$ of inequivalent cusps of $\Gamma$. $R(\Gamma)$ is usually referred as the cusps of $\Gamma$.

In this thesis we will work with the following level $N$ congruence subgroup:

$$
\Gamma_{0}(N)=\left\{\left(\begin{array}{ll}
a & b \\
c & d
\end{array}\right) \in S L_{2}(\mathbb{Z}) \mid c \equiv 0 \quad(\bmod N)\right\}
$$


In the next example, we introduce a matrix in $\Gamma_{0}(N)$, which will come in handy in the upcoming sections.

Example 2.2.2. The matrix $T=\left(\begin{array}{ll}1 & 1 \\ 0 & 1\end{array}\right) \in \Gamma_{0}(N)$ will play an important role in the upcoming section. $T$ acts on $z \in \mathbb{C}$ as follows:

$$
T(z)= \begin{cases}z+1 & \text { if } z \neq \infty \\ \infty & \text { if } z=\infty\end{cases}
$$

That is all cusps with difference 1 are equivalent cusps for $\Gamma_{0}(N)$. In particular, there is always a set $R\left(\Gamma_{0}(N)\right)$ of representatives of inequivalent cusps of $\Gamma_{0}(N)$ in the interval $(0,1]$.

The set $R\left(\Gamma_{0}(N)\right)$ is very important to the understanding of the behaviour of modular forms. In [34, Proposition 2.6] and [48] an example of $R\left(\Gamma_{0}(N)\right)$ is given as follows.

Theorem 2.2.3. A set of representatives from each equivalence class of cusps for $\Gamma_{0}(N)$ is given by $R\left(\Gamma_{0}(N)\right)=\bigcup_{c \mid N}\left\{\frac{a}{c} \mid 1 \leq a \leq N, \operatorname{gcd}(a, c)=1\right.$ and $\left.a \not \equiv a^{\prime}(\bmod \operatorname{gcd}(c, N / c))\right\}$ Note that $R\left(\Gamma_{0}(N)\right)$ is not unique. In our computations we will choose $R\left(\Gamma_{0}(N)\right)$, in such a way that $\frac{1}{c} \in R\left(\Gamma_{0}(N)\right)$, for all $c \mid N$. Let $\phi(n)$ be Euler's totient function. Then $\left|R\left(\Gamma_{0}(N)\right)\right|=\sum_{1 \leq c \mid N} \phi(\operatorname{gcd}(N / c, c))$. 
Example 2.2.4. Let $N=36$, then

$$
\begin{aligned}
R\left(\Gamma_{0}(36)\right) & =\bigcup_{c \mid 36}\left\{\frac{a}{c} \mid \operatorname{gcd}(a, c)=1 \text { and } a \not \equiv a^{\prime}(\bmod \operatorname{gcd}(c, 36 / c))\right\} \\
& =\left\{\frac{1}{1}, \frac{1}{2}, \frac{1}{3}, \frac{2}{3}, \frac{1}{4}, \frac{1}{6}, \frac{5}{6}, \frac{1}{9}, \frac{1}{12}, \frac{5}{12}, \frac{1}{18}, \frac{1}{36}\right\} .
\end{aligned}
$$

In the next example we find an $h \in \mathbb{N}$, such that $M T^{h} M^{-1} \in \Gamma_{0}(N)$ for all $M \in S L_{2}(\mathbb{Z})$. This result will be used to explain the holomorphic condition of the definition of a modular form in the next section.

Example 2.2.5. Let $N \in \mathbb{N}$ and $M=\left(\begin{array}{ll}a & b \\ c & d\end{array}\right) \in S L_{2}(\mathbb{Z})$, with $c \mid N$. Consider the matrix multiplication

$$
M T^{h} M^{-1}=\left(\begin{array}{cc}
1-a c h & a^{2} h \\
-c^{2} h & 1+a c h
\end{array}\right)
$$

If we let $h=\frac{N}{\operatorname{gcd}\left(N, c^{2}\right)}$, then $N \mid-c^{2} h$, i.e. $M T^{h} M^{-1} \in \Gamma_{0}(N)$. In general the minimal $h$ with $M T^{h} M^{-1} \in \Gamma$, is called the width of the cusp $M(\infty)=\frac{a}{c}$. For cusp width see, [28, p. 74].

\subsection{Modular Forms}

This section follows [40, Section 1.4], with the weight restricted to be an integer, the modular subgroup to be $\Gamma_{0}(N)$ and the multiplier system to be a real character.

Definition 2.3.1. 40 , p. 15] Let $k, N \in \mathbb{N}$ and $\chi$ be a real Dirichlet char- 
acter. A function $f(z): \mathbb{H} \rightarrow \mathbb{C}$ is called a modular form of weight $k$ and multiplier system $\chi$ for $\Gamma_{0}(N)$ if $f(z)$ is holomorphic on $\mathbb{H}$ and has the following two properties:

(i) The relation

$$
f(L z)=f\left(\frac{a z+b}{c z+d}\right)=\chi(d)(c z+d)^{k} f(z)
$$

holds for every $L=\left(\begin{array}{ll}a & b \\ c & d\end{array}\right) \in \Gamma_{0}(N)$.

(ii) The function $f(z)$ is holomorphic at all cusps $r \in \mathbb{Q} \cup\{\infty\}$.

We use $M_{k}\left(\Gamma_{0}(N), \chi\right)$ to denote the weight $k$ modular form space for $\Gamma_{0}(N)$ with multiplier system $\chi$, and $M_{k}\left(\Gamma_{0}(N)\right)$ is commonly used when $\chi=\chi_{1}$.

Assume that the function $f(z): \mathbb{H} \rightarrow \mathbb{C}$ satisfies the transformation condition of Definition 2.3.1 (i). Now following [40, Section 1.4], we explain the holomorphic condition of Definition 2.3.1 (ii) for $M_{k}\left(\Gamma_{0}(N), \chi\right)$. First we explain it for the cusp $\infty$. Since $T=\left(\begin{array}{ll}1 & 1 \\ 0 & 1\end{array}\right) \in \Gamma_{0}(N)$, using Definition 2.3 .1 (i) we obtain

$$
f(z+1)=\chi(1) f(z)=f(z) .
$$

So $f(z)$ is a holomorphic function with period 1 on upper half plane. Hence $f(z)$ can be written as a holomorphic function of the variable $q=e^{2 \pi i z}$ in the punctured unit disc, which therefore has a Laurent expansion valid for 
$0<|q|<1$ :

$$
f(z)=\sum_{n=-\infty}^{\infty} a_{n} q^{n} .
$$

Definition 2.3.2. The smallest integer $n$, such that $a_{n} \neq 0$ is said to be the order of $f(z)$ at cusp $\infty$ and denoted by $v_{\infty}(f(z))$. In this notation we tend to drop $f(z)$, whenever the function is clear from the context.

If $v_{\infty}(f(z)) \geq 0$, then the function $f(z)$ is holomorphic at the cusp $\infty$, i.e. $a_{n}=0$ for all $n<0$. In this case we refer to $a_{0}$ as the first term of the expansion of $f(z)$ at cusp $\infty$.

Now let us explain Definition 2.3.1 (ii) for the cusps $r \in \mathbb{Q}$. We write $r=\frac{a}{c}$ with $\operatorname{gcd}(a, c)=1$. Then

$$
r=A(\infty) \text { for some } A=\left(\begin{array}{ll}
a & b \\
c & d
\end{array}\right) \in \Gamma_{0}(1)=S L_{2}(\mathbb{Z})
$$

Let $h$ be minimal such that $N \mid c^{2} h$, then we have $A T^{h} A^{-1}=\left(\begin{array}{cc}1-a c h & a^{2} h \\ -c^{2} h & 1+a c h\end{array}\right) \in$ $\Gamma_{0}(N)$. Define the holomorphic function

$$
g(z)=e(-\kappa z)(A(h z)-r)^{k} f(A(h z)) .
$$

with $0 \leq \kappa<1$ such that

$$
e^{2 \pi i \kappa}=\chi(1+a c h) .
$$


The $\kappa$ defined by (2.3.1) is called the cusp parameter (or Drehrest) for the cusp $r$, see [40, p. 16]. Since $\chi$ is a real character and $\operatorname{gcd}(1+a c h, N)=1$, we have

$$
\chi(1+a c h)= \pm 1, \text { that is } \kappa=0, \text { or } \frac{1}{2} .
$$

Assuming the transformation formula in Definition 2.3.1(i) holds for $f(z)$, we have

$$
\begin{aligned}
g(T z)= & e(-\kappa T z)(A(h T z)-r)^{k} f(A(h T z)) \\
= & e(-\kappa(z+1))(A(h(z+1))-r)^{k} f(A(h(z+1))) \\
= & e(-\kappa(z+1))(A(h z+h)-r)^{k} f\left(A T^{h}(h z)\right) \\
= & e(-\kappa(z+1))(A(h z+h)-r)^{k} f\left(A T^{h} A^{-1} A(h z)\right) \\
= & e(-\kappa(z+1))(A(h z+h)-r)^{k} \\
& \times e(\kappa)\left(-c^{2} h A(h z)+(1+a c h)\right)^{k} f\left(A T^{h}(h z)\right) \\
= & e(-\kappa z)(A(h z)-r)^{k} f\left(A T^{h}(h z)\right) \\
= & g(z) .
\end{aligned}
$$

That is, $g(z)$ is a periodic function with period 1 . Hence $g(z)$ has a Fourier series expansion in the following form:

$$
g(z)=e(-\kappa z)(A(h z)-r)^{k} f(A(h z))=\sum_{n \in \mathbb{Z}} c_{n} e^{2 \pi i n z} .
$$


Then for $f(z)$ we have

$$
f(A(h z))=(A(h z)-r)^{-k} \sum_{n \in \mathbb{Z}} c_{n} e^{2 \pi i(n+\kappa) z} .
$$

Replacing $z$ with $\frac{A^{-1}(z)}{h}$ in 2.3 .3 , we have a Fourier expansion of $f(z)$ at cusp $r$

$$
f(z)=(z-r)^{-k} \sum_{n \in \mathbb{Z}} c_{n} e^{\frac{2 \pi i(n+\kappa) A^{-1}(z)}{h}}
$$

which is equation (1.18) in 40].

Definition 2.3.3. Let $n$ be the smallest integer such that $c_{n} \neq 0$ in (2.3.4). Then $n+\kappa$ is said to be the order of $f(z)$ at cusp $r$ and denoted by $v_{r}(f(z))$. As noted in Definition 2.3.2, in this notation we tend to drop $f(z)$, whenever the function is clear from the context.

If $v_{r}(f(z)) \geq 0$, then the function $f(z)$ is holomorphic at the cusp $r$, i.e. $c_{n}=0$ for all $n<0$. In this case we refer to $(z-r)^{-k} c_{0}$ as the first term of expansion of $f(z)$ at cusp $r$.

Remark 2.3.4. [70, Proposition 1.16] If $f(z)$ is holomorphic at a cusp $r \in$ $\mathbb{Q} \cup\{\infty\}$, then it is holomorphic at all cusps equivalent to $r$ in $\Gamma_{0}(N)$. That is provided $f(z)$ satisfies Definition 2.3.1 (i), to show that $f(z)$ is a modular form we need to check condition Definition 2.3.1(ii) for elements of $R\left(\Gamma_{0}(N)\right)$, instead of all $r \in \mathbb{Q} \cup\{\infty\}$. 
From Definitions 2.3.1, 2.3.2, 2.3.3 and equations 2.3 .2 and 2.3 .4 we deduce the following theorem.

Theorem 2.3.5. Let $f(z) \in M_{k}\left(\Gamma_{0}(N), \chi\right)$, with $\chi$ a primitive real character whose modulus is dividing $N$, and let $r=\frac{a}{c} \in R\left(\Gamma_{0}(N)\right)$ then

$$
v_{r}(f(z)) \in \begin{cases}\mathbb{N}_{0} & \text { if } \operatorname{gcd}\left(c, \frac{N}{c}\right)=1, \\ \mathbb{N}_{0} & \text { if } \operatorname{gcd}\left(c, \frac{N}{c}\right)>1 \text { and } \chi\left(1+\frac{a N}{\operatorname{gcd}\left(c, \frac{N}{c}\right)}\right)=1, \\ \mathbb{N}_{0}+\frac{1}{2} & \text { if } \operatorname{gcd}\left(c, \frac{N}{c}\right)>1 \text { and } \chi\left(1+\frac{a N}{\operatorname{gcd}\left(c, \frac{N}{c}\right)}\right)=-1 .\end{cases}
$$

Definition 2.3.6. If $f(z)$ is a modular form and $v_{r}(f(z))>0$ for all $r \in$ $\mathbb{Q} \cup\{\infty\}$, then we call $f(z)$ a cusp form.

We use $S_{k}\left(\Gamma_{0}(N), \chi\right)$ to denote the weight $k$ cusp form space for $\Gamma_{0}(N)$ with multiplier system $\chi$. It is also clear that we have $S_{k}\left(\Gamma_{0}(N), \chi\right) \subset$ $M_{k}\left(\Gamma_{0}(N), \chi\right)$.

Remark 2.3.7. It is well known that modular form spaces are vector spaces, furthermore we have the following decomposition

$$
M_{k}\left(\Gamma_{0}(N), \chi\right)=E_{k}\left(\Gamma_{0}(N), \chi\right) \oplus S_{k}\left(\Gamma_{0}(N), \chi\right)
$$

where $E_{k}\left(\Gamma_{0}(N), \chi\right)$ is the Eisenstein subspace, which is uniquely determined by this decomposition, see [51, Theorem 2.1.7].

For notational convenience we write $S_{k}\left(\Gamma_{0}(N)\right)$ instead of $S_{k}\left(\Gamma_{0}(N), \chi_{1}\right)$ and $E_{k}\left(\Gamma_{0}(N)\right)$ instead of $E_{k}\left(\Gamma_{0}(N), \chi_{1}\right)$. 


\subsection{Some Properties of Modular Forms}

The following statements are immediate from Definition 2.3.1.

Remark 2.4.1. Let $f(z) \in M_{k}\left(\Gamma_{0}(N), \chi\right)$, then for all $t \in \mathbb{N}$, we have $f(z) \in M_{k}\left(\Gamma_{0}(t N), \chi\right)$, i.e. $M_{k}\left(\Gamma_{0}(N), \chi\right) \subseteq M_{k}\left(\Gamma_{0}(t N), \chi\right)$.

Remark 2.4.2. Let $f(z), g(z) \in M_{k}\left(\Gamma_{0}(N), \chi\right)$, then for all $a, b \in \mathbb{Q}$, we have $a f(z)+b g(z) \in M_{k}\left(\Gamma_{0}(N), \chi\right)$.

Remark 2.4.3. Let $f(z) \in M_{k}\left(\Gamma_{0}(N), \chi\right)$, then for all $t \in \mathbb{N}$, we have $f(t z) \in M_{k}\left(\Gamma_{0}(\operatorname{lcm}(t, N)), \chi\right)$.

We use the Sturm bound $S(2 k, N)$ defined by 2.4.1) to show the equality of two modular forms in the same modular space. The following theorem is the special case for $M_{2 k}\left(\Gamma_{0}(N)\right)$, see [36, Theorem 3.13] for the general case.

Theorem 2.4.4. Let $f(z), g(z) \in M_{2 k}\left(\Gamma_{0}(N)\right)$ have the Fourier series expansions

$$
f(z)=\sum_{n=0}^{\infty} a_{n} q^{n} \text { and } g(z)=\sum_{n=0}^{\infty} b_{n} q^{n} .
$$

The Sturm bound $S(2 k, N)$ for the modular space $M_{2 k}\left(\Gamma_{0}(N)\right)$ is given by

$$
S(2 k, N)=\frac{N k}{6} \prod_{p \mid N}(1+1 / p)
$$

and so if $a_{n}=b_{n}$ for all $n \leq S(2 k, N)$ then $f(z)=g(z)$. 


\section{Chapter 3}

\section{Eisenstein Series}

In this chapter we introduce Eisenstein series and use them to generate $E_{k}\left(\Gamma_{0}(N), \chi\right),(k, N \in \mathbb{N}), \chi$ a primitive character (not necessarily real) with conductor dividing $N$. In the first section we introduce generalized Bernoulli numbers, which appear as the first terms of series expansions of Eisenstein series. In the second section we give the definition of the Eisenstein series along with criteria to determine their modularity and a theorem to determine bases for $E_{k}\left(\Gamma_{0}(N), \chi\right)$, for all $k, N \in \mathbb{N}, \chi$ a primitive character (not necessarily real) with conductor dividing $N$. All these results were previously known. 


\subsection{Bernoulli Numbers}

In this section we introduce Bernoulli numbers and generalized Bernoulli numbers. We then give values of certain generalized Bernoulli numbers as an example.

Definition 3.1.1. (Generalized Bernoulli Number, [70, Definition 5.1] ) Let $k, N \in \mathbb{N}$ and $\chi$ be a primitive character of modulus $N$. We define the Bernoulli numbers, $B_{k}$ and generalized Bernoulli numbers $B_{k, \chi}$ attached to $\chi$ by the following equations, respectively:

$$
\begin{aligned}
& \sum_{k=0}^{\infty} B_{k} \frac{x^{k}}{k !}=\frac{x}{e^{x}-1} \\
& \sum_{k=0}^{\infty} B_{k, \chi} \frac{x^{k}}{k !}=\sum_{a=1}^{N} \frac{\chi(a) x e^{a x}}{e^{N x}-1} .
\end{aligned}
$$

Note that

$$
\begin{aligned}
& B_{2, \chi_{1}}=-B_{2}, \\
& B_{k, \chi_{1}}=B_{k}, \text { when } k \neq 2 .
\end{aligned}
$$

The $B_{k}$ are sometimes called the first Bernoulli numbers and the $B_{k, \chi_{1}}$ as the second Bernoulli numbers.

Example 3.1.2. Let us compute $B_{k, \chi_{12}}$, for $k \leq 6$. By Definition 3.1.1 we 
have

$$
\sum_{a=1}^{12} \frac{\chi_{12}(a) x e^{a x}}{e^{12 x}-1}=\sum_{k=0}^{\infty} B_{k, \chi_{12}} \frac{x^{k}}{k !}
$$

That is

$$
B_{k, \chi_{12}}=k ![k] \sum_{a=1}^{12} \frac{\chi_{12}(a) x e^{a x}}{e^{12 x}-1} .
$$

We use MAPLE to expand the sum on right hand side of the equation and find

$$
\sum_{a=1}^{12} \frac{\chi_{12}(a) x e^{a x}}{e^{12 x}-1}=2 x^{2}-\frac{23}{3} x^{4}+\frac{1681}{60} x^{6}+O\left(x^{7}\right) .
$$

Hence we have

$$
\begin{aligned}
& B_{1, \chi_{12}}=0, B_{2, \chi_{12}}=4, B_{3, \chi_{12}}=0 \\
& B_{4, \chi_{12}}=-184, B_{5, \chi_{12}}=0, B_{6, \chi_{12}}=20172 .
\end{aligned}
$$

\subsection{Eisenstein Series}

In this section we define Eisenstein series, which are one of the central objects of this thesis. We can write any modular form in $E_{k}\left(\Gamma_{0}(N), \chi\right)$, for any $k \geq 1$, $N \geq 1$ and $\chi$ a character with modulus dividing $N$, not necessarily real, as linear combinations of Eisenstein series, see [70, Theorem 5.9]. Let $\chi$ and 
$\psi$ be primitive Dirichlet characters (not necessarily real) with conductors $L \in \mathbb{N}$ and $R \in \mathbb{N}$, respectively.

We note that in classical literature Eisenstein series are defined as summation of fractions over lattice points, see [51, (7.1.1), p. 268], [15, Section 1.9]. In this thesis we use Fourier series expansions of Eisenstein series at infinity as definitions, the interested reader may see [51, Section 7.1] to see how their series expansions at infinity is obtained. Later on, in Chapter 6 , we will give Fourier series expansions of a family of these Eisenstein series at cusps $\frac{1}{c},(c \in \mathbb{N})$.

For $n \in \mathbb{N}$ we define $\sigma_{(k, \chi, \psi)}(n)$ by

$$
\sigma_{(k, \chi, \psi)}(n)=\sum_{1 \leq d \mid n} \chi(d) \psi(n / d) d^{k}
$$

If $n \notin \mathbb{N}$ we set $\sigma_{(k, \chi, \psi)}(n)=0$. Note that $\sigma_{\left(k, \chi_{1}, \chi_{1}\right)}(n)$ coincides with the sum of divisors function

$$
\sigma_{k}(n)=\sum_{1 \leq m \mid n} m^{k}
$$

Also note that we write $\sigma(n)$ for $\sigma_{k}(n)$ when $k=1$. We define the Eisenstein series by

$$
E_{k, \chi, \psi}(z)=c_{0}+\sum_{n \geq 1} \sigma_{(k-1, \chi, \psi)}(n) q^{n}
$$


where

$$
c_{0}=\left\{\begin{aligned}
-\frac{B_{k}}{2 k} & \text { if } \chi=\psi=\chi_{1}, \\
-\frac{B_{k, \chi}}{2 k} & \text { if } R=1 \text { and } L>1 \\
0 & \text { if } R>1
\end{aligned}\right.
$$

Note that the Eisenstein series $E_{2 k, \chi_{1}, \chi_{1}}(z)$, coincides with the Eisenstein series $E_{2 k}(z)$ defined by

$$
E_{2 k}(z)=-\frac{B_{2 k}}{4 k}+\sum_{n \geq 1} \sigma_{2 k-1}(n) q^{n} .
$$

To read more about this special case and to see how $E_{4}(z)$ and $E_{6}(z)$ generate $M_{2 k}\left(\Gamma_{0}(1)\right)$ for all $k \in \mathbb{N}$, see [36, Proposition 3.6].

The following two statements are taken from [70], who excellently derived the statements from [51].

Theorem 3.2.1. ([70, Theorem 5.8]) Suppose $t$ is a positive integer and $\chi$ and $\psi$ be primitive Dirichlet characters (not necessarily real) with conductors $L \in \mathbb{N}$ and $R \in \mathbb{N}$, respectively, and that $k \in \mathbb{N}$ is such that $\chi(-1) \psi(-1)=$ $(-1)^{k}$. Then we have

$$
\begin{aligned}
& E_{2}(z)-t E_{2}(t z) \in E_{2}\left(\Gamma_{0}(t)\right) \text { and } \\
& E_{k, \chi, \psi}(t z) \in E_{k}\left(\Gamma_{0}(R L t), \chi \psi\right) \text { if } R>1 \text { or } L>1 .
\end{aligned}
$$


For notational convenience we write

$$
L_{t}(z)=E_{2}(z)-t E_{2}(t z)
$$

Theorem 3.2.2. ([70, Theorem 5.9]) Suppose $k, N \in \mathbb{N}$. Let $\chi$ be a primitive character with modulus dividing $N$ and $\chi(-1)=(-1)^{k}$. Let $\varepsilon$ and $\psi$ are primitive Dirichlet characters (not necessarily real) with conductors $L, R \in$ $\mathbb{N}$, respectively, with $L, R \mid N$. Then the following sets of Eisenstein series form a basis for $E_{k}\left(\Gamma_{0}(N), \chi\right)$

$$
\begin{aligned}
& \text { (i) }\left\{L_{t}(z)|1<t| N\right\} \\
& \quad \cup\left\{E_{2, \varepsilon, \psi}(t z)\left|\varepsilon \psi=\chi_{1}, R L t\right| N \text { and } \varepsilon, \psi \neq \chi_{1}\right\} \text { if } k=2 \text {, and } \chi=\chi_{1} \\
& \text { (ii) }\left\{E_{k, \varepsilon, \psi}(t z) \mid \varepsilon \psi=\chi \text { and } R L t \mid N\right\} \quad \text { otherwise. }
\end{aligned}
$$

Note that $\varepsilon \psi=\chi$, means the values $\varepsilon(n) \psi(n)$ and $\chi(n)$ agree whenever $\operatorname{gcd}(n, N)=1$.

\subsection{Bases of $E_{k}\left(\Gamma_{0}(N)\right)$ for a Family of $N$ 's}

In this section we give bases for $E_{2 k}\left(\Gamma_{0}(N)\right)$, for all $k \in \mathbb{N}$ and $N=$ $N_{1}, 2 N_{1}, 4 N_{1}$ for a squarefree number $N_{1} \in \mathbb{N}$. $N$ defined this way is special, because for all $c \mid N$, we have $\phi(\operatorname{gcd}(c, N / c))=1$.

Corollary 3.3.1. Let $k>1$ be an integer and $N_{1} \in \mathbb{N}$ be a square free 
number. Then for $N=N_{1}, 2 N_{1}, 4 N_{1}$ the set of Eisenstein series

$$
\begin{aligned}
& \left\{E_{2}(z)-t E_{2}(t z)|1<t| N\right\}, \\
& \left\{E_{2 k}(t z)|1 \geq t| N\right\}
\end{aligned}
$$

forms a basis for $E_{2}\left(\Gamma_{0}(N)\right)$ and $E_{2 k}\left(\Gamma_{0}(N)\right)$, respectively.

Proof. Let $N_{1} \in \mathbb{N}$ be a square free number and let $N=N_{1}, 2 N_{1}$, or $4 N_{1}$. Let $\varepsilon$ and $\psi$ be primitive Dirichlet characters (not necessarily real) with conductors $L, R \in \mathbb{N}$, respectively, with $L, R \mid N$. If $\varepsilon \psi=\chi_{1}$, then $R=L$. Thus, we have either $R=L=1, R=L=2$, or $R L \nmid N$. There are no primitive Dirichlet characters with conductor 2 . Then for all $k>1$, $\left\{E_{k, \varepsilon, \psi}(t z)\left|\varepsilon \psi=\chi_{1}, R L t\right| N\right.$ and $\left.\varepsilon, \psi \neq \chi_{1}\right\}$ is an empty set, hence the result follows by Theorem 3.2 .2 .

As an example to Corollary 3.3.1 we give a basis for $E_{2 k}\left(\Gamma_{0}(12)\right)$ for $k>1$. We use this basis in Chapter 7 , where we give an extension to the Ramanujan-Mordell formula.

Example 3.3.2. Let $k \in \mathbb{N}$. By Corollary 3.3.1, the set

$$
\left\{E_{2 k}(z), E_{2 k}(2 z), E_{2 k}(3 z), E_{2 k}(4 z), E_{2 k}(6 z), E_{2 k}(12 z)\right\}
$$

is a basis for $E_{2 k}\left(\Gamma_{0}(12)\right)$ for all $k>1$, where the Eisenstein series are given 
as

$$
E_{2 k}(t z)=\frac{-B_{k}}{2 k}+\sum_{n \geq 1} \sigma_{2 k-1}(n / t) q^{n}, \text { for } t=1,2,3,4,6,12
$$




\section{Chapter 4}

\section{Eta Quotients}

In this chapter, we introduce the Dedekind eta function and eta quotients. The first section of this chapter presents results from [40], where we give statements for transformation formula and series expansion at each cusp of the Dedekind eta function. Using these we give a formula for orders of eta quotients at each cusp and criteria to determine if an eta quotient is a modular form. These criteria and formula will be used throughout the thesis. In the second section we play with (4.1.4) to show that if two eta quotients have the same 'level' then their sum of orders are the same. This fact will be useful in the third and fourth sections, where we find lists of eta quotients in $M_{2}\left(\Gamma_{0}(16), \chi_{8}\right)$ and $M_{2}\left(\Gamma_{0}(N)\right)$ for all $N$ which are multiples of two distinct primes. We finish the chapter by giving the number of eta quotients in $M_{2}\left(\Gamma_{0}(N)\right)$ for all $N \leq 100$. 


\subsection{Dedekind Eta Function}

Recall that the Dedekind eta function $\eta(z)$ is the holomorphic function defined on the upper half plane $\mathbb{H}=\{z \in \mathbb{C} \mid \operatorname{Im}(z)>0\}$ by the product formula

$$
\eta(z)=e^{\pi i z / 12} \prod_{n=1}^{\infty}\left(1-e^{2 \pi i n z}\right) .
$$

Let $N \in \mathbb{N}$ and $r_{\delta} \in \mathbb{Z}$ for all positive divisors $\delta$ of $N$. Let $z \in \mathbb{H}$. We define an eta quotient by the product formula

$$
f(z)=\prod_{1 \leq \delta \mid N} \eta^{r_{\delta}}(\delta z)
$$

Recall that we use the notation $q=e(z)=e^{2 \pi i z}$ and since $z \in \mathbb{H}$, we have $|q|<1$.

Let $\delta_{1}, \delta_{2}, \ldots, \delta_{\sigma_{0}(N)}$ be the divisors of $N$. For convenience, sometimes we use the following shorthand notation

$$
\eta_{N}\left[r_{\delta_{1}}, r_{\delta_{2}}, \ldots, r_{\delta_{\sigma_{0}(N)}}\right](z)=\eta^{r_{\delta_{1}}}\left(\delta_{1} z\right) \eta^{r_{\delta_{2}}}\left(\delta_{2} z\right) \ldots \eta^{r_{\sigma_{0}(N)}}\left(\delta_{\sigma_{0}(N)} z\right) .
$$

Let $L=\left(\begin{array}{ll}a & b \\ c & d\end{array}\right) \in S L_{2}(\mathbb{Z})$ and $\left(\frac{c}{d}\right)^{*},\left(\frac{c}{d}\right)_{*}$ be defined as in [40, p. 14] (differs from the Kronecker symbol $\left(\frac{c}{d}\right)$ by sign). It is well known that the Dedekind eta function given in (4.1.1) satisfy the transformation formula, see 
[15, Theorem 3.4] and [40, Theorem 1.7] and [38, Section 3.3],

$$
\eta(L z)=v_{\eta}(L)(c z+d)^{1 / 2} \eta(z)
$$

where

$v_{\eta}(L)= \begin{cases}\left(\frac{d}{c}\right)^{*} e\left(\frac{2 \pi i}{24}\left(c(a+d)-b d\left(c^{2}-1\right)-3 c\right)\right) & \text { if } c \text { is odd }, \\ \left(\frac{c}{d}\right)_{*} e\left(\frac{2 \pi i}{24}\left(c(a+d)-b d\left(c^{2}-1\right)+3 d-3-3 c d\right)\right) & \text { if } c \text { is even. }\end{cases}$

Let $\eta_{t}(z)=\eta(t z)$ and $\gamma=\left(\begin{array}{cc}a & b \\ c & d\end{array}\right) \in \Gamma_{0}(N)$. Then for all $t \mid N$, we have $\gamma_{t}=\left(\begin{array}{cc}a & b t \\ c / t & d\end{array}\right) \in S L_{2}(\mathbb{Z})$. Using 4.1.3 we obtain

$$
\eta_{t}(\gamma z)=\eta\left(\frac{a(t z)+b t}{c / t(t z)+d}\right)=\eta\left(\gamma_{t} z\right)=v\left(\gamma_{t}\right)(c z+d)^{1 / 2} \eta_{t}(z)
$$

From [40, Proposition 2.1] we obtain the following theorem.

Theorem 4.1.1. Let $t \in \mathbb{N}$ and let $\eta_{t}(z)=\eta(t z)$ be the Dedekind eta function defined by 4.1 .1 and let $r=\frac{-d}{c} \in \mathbb{Q}$ be a reduced fraction with $c \neq 0$. Let $a, b$ be chosen such that

$$
A=\left(\begin{array}{ll}
a & b \\
c & d
\end{array}\right) \in S L_{2}(\mathbb{Z})
$$

Let $g=\operatorname{gcd}(t, c)$ and let $v_{t}$ and $y_{t} \in \mathbb{Z}$ be such that $\frac{-t v_{t}}{g}+\frac{c y_{t}}{g}=1$. Then 
the Fourier series expansion of $\eta_{t}(z)$ at cusps $r \in \mathbb{Q}$ is given by

$$
\eta_{t}\left(A^{-1} z\right)=v_{\eta}(L)\left(\frac{g}{t}(-c z+a)\right)^{1 / 2} \times \sum_{n \geq 1}\left(\frac{12}{n}\right) \exp \left(\frac{n^{2}}{24 t}\left(g^{2} z+v g\right)\right)
$$

where $L=\left(\begin{array}{ll}-t / g & * \\ -c / g & *\end{array}\right) \in S L_{2}(\mathbb{Z})$.

Since the first non-vanishing term in $\eta_{t}\left(A^{-1} z\right)$ is a constant multiple of $\exp \left(\frac{n^{2}}{24 t}\left(g^{2} z+v g\right)\right)$, by Definitions 2.3 .2 and 2.3.3, we obtain the following statement.

Corollary 4.1.2. The order of $\eta_{t}(z)$ at the cusp $r=\frac{-d}{c} \in \mathbb{Q}$ is

$$
v_{r}\left(\eta_{t}(z)\right)=\frac{1}{24 t} g^{2}
$$

Let $f(z)$ be an eta quotient given by 4.1.2. By Corollary 4.1.2, we have

$$
v_{a / c}(f)=\frac{N}{24 \operatorname{gcd}\left(c^{2}, N\right)} \sum_{1 \leq \delta \mid N} \frac{\operatorname{gcd}(\delta, c)^{2} \cdot r_{\delta}}{\delta}
$$

see also [56, p. 320] and [46, Proposition 3.2.8, p. 34].

\subsection{Eta Quotients}

We start this section by proving Lemmas 4.2.1, 4.2.2 and 4.2.3 which will help us to give an elementary proof for the equation in 4.2.9. 
Lemma 4.2.1. Let $N \in \mathbb{N}$, and $p \| N$ be a prime and $\lambda \mid \frac{N}{p}$. Then for all $c \mid \frac{N}{p}$ we have

$$
\begin{aligned}
& \frac{\phi(\operatorname{gcd}(c, N / c)) \cdot \operatorname{gcd}(c, \lambda)}{\operatorname{gcd}(c, N / c) \cdot \operatorname{lcm}(c, \lambda)}=\frac{\phi(\operatorname{gcd}(c p, N / c p)) \cdot \operatorname{gcd}(c p, p \lambda)}{\operatorname{gcd}(c p, N / c p) \cdot \operatorname{lcm}(c p, p \lambda)}, \\
& \frac{\phi(\operatorname{gcd}(c p, N / c p)) \cdot \operatorname{gcd}(c p, \lambda)}{\operatorname{gcd}(c p, N / c p) \cdot \operatorname{lcm}(c p, \lambda)}=\frac{\phi(\operatorname{gcd}(c, N / c)) \cdot \operatorname{gcd}(c, p \lambda)}{\operatorname{gcd}(c, N / c) \cdot \operatorname{lcm}(c, p \lambda)} .
\end{aligned}
$$

Proof. Since $c \mid \frac{N}{p}$, we have $\operatorname{gcd}(c, p)=1$ and since $p \| N$ we have $\operatorname{gcd}\left(p, \frac{N}{c p}\right)=1$. Using these we have

$\operatorname{gcd}\left(c p, \frac{N}{c p}\right)=\operatorname{gcd}\left(c, \frac{N}{c p}\right) \cdot \operatorname{gcd}\left(p, \frac{N}{c p}\right)=\operatorname{gcd}\left(c, \frac{N}{c p}\right)=\operatorname{gcd}\left(c, \frac{N}{c}\right)$

Then we have

$\frac{\phi(\operatorname{gcd}(c p, N / c p)) \cdot \operatorname{gcd}(c p, p \lambda)}{\operatorname{gcd}(c p, N / c p) \cdot \operatorname{lcm}(c p, p \lambda)}=\frac{\phi(\operatorname{gcd}(c, N / c)) \cdot p \cdot \operatorname{gcd}(c, \lambda)}{\operatorname{gcd}(c, N / c) \cdot p \cdot \operatorname{lcm}(c, \lambda)}=\frac{\phi(\operatorname{gcd}(c, N / c)) \cdot \operatorname{gcd}(c, \lambda)}{\operatorname{gcd}(c, N / c) \cdot \operatorname{lcm}(c, \lambda)}$

The second equation can be proven similarly.

Lemma 4.2.2. Let $N \in \mathbb{N}$, and $p$ be a prime such that $p^{s} \| N$ with $s>1$ and $\lambda \mid \frac{N}{p}$. Then for all $c \mid \frac{N}{p^{s}}$, we have

$$
\begin{aligned}
\frac{\phi\left(\operatorname{gcd}\left(c, \frac{N}{c}\right)\right) \cdot \operatorname{gcd}(c, \lambda)}{\operatorname{gcd}\left(c, \frac{N}{c}\right) \cdot \operatorname{lcm}(c, \lambda)}= & \frac{\phi\left(\operatorname{gcd}\left(c, \frac{N}{c}\right)\right) \cdot \operatorname{gcd}(c, p \lambda)}{\operatorname{gcd}\left(c, \frac{N}{c}\right) \cdot \operatorname{lcm}(c, p \lambda)} \\
& +\frac{\phi(\operatorname{gcd}(c p, N / c p)) \cdot \operatorname{gcd}(c p, p \lambda)}{\operatorname{gcd}(c p, N / c p) \cdot \operatorname{lcm}(c p, p \lambda)},
\end{aligned}
$$




$$
\begin{aligned}
\frac{\phi\left(\operatorname{gcd}\left(c p^{s}, N / c p^{s}\right)\right) \cdot \operatorname{gcd}\left(c p^{s}, p \lambda\right)}{\operatorname{gcd}\left(c p^{s}, N / c p^{s}\right) \cdot \operatorname{lcm}\left(c p^{s}, p \lambda\right)}= & \frac{\phi\left(\operatorname{gcd}\left(c p^{s-1}, N / c p^{s-1}\right)\right) \cdot \operatorname{gcd}\left(c p^{s-1}, \lambda\right)}{\operatorname{gcd}\left(c p^{s-1}, N / c p^{s-1}\right) \cdot \operatorname{lcm}\left(c p^{s-1}, \lambda\right)} \\
& +\frac{\phi\left(\operatorname{gcd}\left(c p^{s}, N / c p^{s}\right)\right) \cdot \operatorname{gcd}\left(c p^{s}, \lambda\right)}{\operatorname{gcd}\left(c p^{s}, N / c p^{s}\right) \cdot \operatorname{lcm}\left(c p^{s}, \lambda\right)},
\end{aligned}
$$

and for all $1 \leq i<s-1$, we have

$$
\frac{\phi\left(\operatorname{gcd}\left(c p^{i}, N / c p^{i}\right)\right) \cdot \operatorname{gcd}\left(c p^{i}, \lambda\right)}{\operatorname{gcd}\left(c p^{i}, N / c p^{i}\right) \cdot \operatorname{lcm}\left(c p^{i}, \lambda\right)}=\frac{\phi\left(\operatorname{gcd}\left(c p^{i+1}, N / c p^{i+1}\right)\right) \cdot \operatorname{gcd}\left(c p^{i+1}, p \lambda\right)}{\operatorname{gcd}\left(c p^{i+1}, N / c p^{i+1}\right) \cdot \operatorname{lcm}\left(c p^{i+1}, p \lambda\right)} .
$$

Proof. Since $c \mid \frac{N}{p^{s}}$ with $s>1$, we have $\operatorname{gcd}(c, p)=1$ and $\operatorname{gcd}(p, N / c p)=p$. Using these we have $\operatorname{gcd}\left(c p, \frac{N}{c p}\right)=\operatorname{gcd}\left(c, \frac{N}{c p}\right) \cdot \operatorname{gcd}\left(p, \frac{N}{c p}\right)=p \cdot \operatorname{gcd}\left(c, \frac{N}{c p}\right)=p \cdot \operatorname{gcd}\left(c, \frac{N}{c}\right)$.

Also since $\operatorname{gcd}\left(p, \operatorname{gcd}\left(c, \frac{N}{c}\right)\right)=1$, we have

$$
\phi\left(\operatorname{gcd}\left(c p, \frac{N}{c p}\right)\right)=\phi\left(p \cdot \operatorname{gcd}\left(c, \frac{N}{c}\right)\right)=(p-1) \cdot \phi\left(\operatorname{gcd}\left(c, \frac{N}{c}\right)\right)
$$

Hence 4.2.1) follows from 4.2.4 and 4.2.5 (4.2.2) can be proven similarly. 
Let $1 \leq i \leq s-1$ then we have

$$
\operatorname{gcd}\left(c p^{i}, \frac{N}{c p^{i}}\right)= \begin{cases}p^{i} \operatorname{gcd}(c, N / c) & \text { if } i<\frac{s}{2} \\ p^{s-i} \operatorname{gcd}(c, N / c) & \text { if } i \geq \frac{s}{2}\end{cases}
$$

We also have $\phi\left(p^{t+1}\right)=p^{t+1}-p^{t}=p \cdot\left(p^{t}-p^{t-1}\right)$ for all $t \geq 1$. Hence the equation 4.2.3 follows by (4.2.6).

From Lemma 4.2.1 and 4.2.2, we deduce the following lemma.

Lemma 4.2.3. Let $N \in \mathbb{N}$ and $p$ be a prime divisor of $N$. Then for all $\lambda \mid \frac{N}{p}$ we have

$$
\sum_{c \mid N} \frac{\phi(\operatorname{gcd}(c, N / c)) \cdot \operatorname{gcd}(c, \lambda)}{\operatorname{gcd}(c, N / c) \cdot \operatorname{lcm}(c, \lambda)}=\sum_{c \mid N} \frac{\phi(\operatorname{gcd}(c, N / c)) \cdot \operatorname{gcd}(c, p \lambda)}{\operatorname{gcd}(c, N / c) \cdot \operatorname{lcm}(c, p \lambda)}
$$

Clearly $1 \mid \frac{N}{p}$ for all $p \mid N$. Then for all $\delta \mid N$ we have

$$
\sum_{c \mid N} \frac{\phi(\operatorname{gcd}(c, N / c))}{\operatorname{gcd}(c, N / c) \cdot c}=\sum_{c \mid N} \frac{\phi(\operatorname{gcd}(c, N / c)) \cdot \operatorname{gcd}(c, \delta)}{\operatorname{gcd}(c, N / c) \cdot \operatorname{lcm}(c, \delta)}
$$

We are now ready to prove an important theorem, which will let us determine a set of eta quotients that contains all eta quotients in $M_{k}\left(\Gamma_{0}(N), \chi\right)$.

Theorem 4.2.4. Let $N, k \in \mathbb{N}$ and $r_{\delta} \in \mathbb{Z}$ for all $\delta \mid N$. Let $f(z)=$ $\prod_{1 \leq \delta \mid N} \eta^{r_{\delta}}(\delta z)$ be an eta quotient. Let $v_{r}(f(z))$ be the order of $f(z)$ at the cusp 
$r \in R\left(\Gamma_{0}(N)\right)$ given by (4.1.4). If $f(z) \in M_{k}\left(\Gamma_{0}(N), \chi\right)$ then

$$
\sum_{r \in R\left(\Gamma_{0}(N)\right)} v_{r}(f(z))=\frac{2 k N}{24} \sum_{c \mid N} \frac{\phi(\operatorname{gcd}(c, N / c))}{\operatorname{gcd}\left(c^{2}, N\right)}
$$

with

$$
v_{r}(f(z)) \in \begin{cases}\mathbb{N}_{0} & \text { if } \operatorname{gcd}(c, N / c)=1, \\ \mathbb{N}_{0} & \text { if } \operatorname{gcd}(c, N / c)>1 \text { and } \chi\left(1+\frac{N}{\operatorname{gcd}(c, N / c)}\right)=1,(4 . \\ \mathbb{N}_{0}+\frac{1}{2} & \text { if } \operatorname{gcd}(c, N / c)>1 \text { and } \chi\left(1+\frac{N}{\operatorname{gcd}(c, N / c)}\right)=-1 .\end{cases}
$$

That is, we have

$$
0 \leq v_{r}(f(z)) \leq \frac{2 k N}{24} \sum_{c \mid N} \frac{\phi(\operatorname{gcd}(c, N / c))}{\operatorname{gcd}\left(c^{2}, N\right)}
$$

Proof. 4.2.10 follows from Theorem 2.3.5. Note that $v_{r}(f(z))$ depends only on the denominator of $r=\frac{a}{c}$. Using Lemma 4.2.3 we obtain

$$
\begin{aligned}
\sum_{r \in R\left(\Gamma_{0}(N)\right)} v_{r}(f(z)) & =\sum_{r \in R\left(\Gamma_{0}(N)\right)} \frac{N}{24 \operatorname{gcd}\left(c^{2}, N\right)} \sum_{1 \leq \delta \mid N} \frac{\operatorname{gcd}(\delta, c)^{2} \cdot r_{\delta}}{\delta} \\
& =\sum_{1 \leq \delta \mid N} \frac{N}{24} \sum_{r \in R\left(\Gamma_{0}(N)\right)} \frac{\operatorname{gcd}(\delta, c)^{2} \cdot r_{\delta}}{\operatorname{gcd}\left(c^{2}, N\right) \cdot \delta} \\
& =\frac{N}{24} \sum_{1 \leq \delta \mid N} r_{\delta} \sum_{c \mid N} \frac{\phi(\operatorname{gcd}(c, N / c)) \cdot \operatorname{gcd}(\delta, c)^{2}}{\operatorname{gcd}\left(c^{2}, N\right) \cdot \delta} \\
& =\frac{N}{24} \sum_{1 \leq \delta \mid N} r_{\delta} \sum_{c \mid N} \frac{\phi(\operatorname{gcd}(c, N / c))}{\operatorname{gcd}\left(c^{2}, N\right)}
\end{aligned}
$$




$$
\begin{aligned}
& =\frac{N}{24} \sum_{c \mid N} \frac{\phi(\operatorname{gcd}(c, N / c))}{\operatorname{gcd}\left(c^{2}, N\right)} \sum_{1 \leq \delta \mid N} r_{\delta} \\
& =\frac{2 k N}{24} \sum_{c \mid N} \frac{\phi(\operatorname{gcd}(c, N / c))}{\operatorname{gcd}\left(c^{2}, N\right)} .
\end{aligned}
$$

Also by condition (2) of Definition 2.3.1, we have $v_{r}(f(z)) \geq 0$. Thus we have the desired inequality in 4.2.11).

Also note that the equality 4.2 .9 can alternatively be proven by using the Hurwitz formula, see [44, p. 30]. For details see the proof of Theorem 11 in 65. From the equality 4.2 .9 we deduce that the sum of cusps of an eta quotient does not depend on the exponents of the Dedekind eta functions, it depends only on $N$.

We note the following remark for future use.

Remark 4.2.5. Let $f(z)$ be an eta quotient given by 4.1.2). Consider the system of equations given by

$$
v_{1 / c}(f)=\frac{N}{24 \operatorname{gcd}\left(c^{2}, N\right)} \sum_{1 \leq \delta \mid N} \frac{\operatorname{gcd}(\delta, c)^{2} \cdot r_{\delta}}{\delta}
$$

for all $c \mid N$. If the coefficient matrix of this system is invertible then the exponents $r_{\delta}(\delta \mid N)$ of the eta quotient are uniquely determined by the orders of $f(z)$ at cusps $1 / c$.

As noted earlier we use Theorem 4.2.4 to determine all possible eta quotients in a given space. We use the following theorem to determine if the 
eta quotients arose from previous theorem are actually in the given space of modular forms. This theorem is referred to as Ligozat's Criteria, see [36, Theorem 5.7, p. 99], [40, Corollary 2.3, p. 37], [30, p. 174], [46], [55] and [47, Proposition 1, p. 284].

Theorem 4.2.6. Let $f(z)$ be an eta quotient given by (4.1.2) which satisfies the following conditions

(L1) $\sum_{1 \leq \delta \mid N} \delta \cdot r_{\delta} \equiv 0(\bmod 24)$

(L2) $\sum_{1 \leq \delta \mid N} \frac{N}{\delta} \cdot r_{\delta} \equiv 0(\bmod 24)$,

(L3) $v_{1 / d}(f(z)) \geq 0$ for each positive divisor $d$ of $N$,

(L4) $k=\frac{1}{2} \sum_{1 \leq \delta \mid N} r_{\delta}$ is a positive integer.

Then $f(z) \in M_{k}\left(\Gamma_{0}(N), \chi\right)$ where the character $\chi$ is given by

$$
\chi(m)=\left(\frac{(-1)^{k} s}{m}\right)
$$

with

$$
s=\prod_{p \mid N} \delta^{\left|\sum_{p \mid \delta} r_{\delta}\right|} .
$$

Furthermore, if the inequalities in (L3) are all strict then $f(z) \in S_{k}\left(\Gamma_{0}(N), \chi\right)$. 
Using the multiplicative properties of the Kronecker symbol given in Definition 2.1.5 we can give a simpler description of the character given by 4.2.12. Let

$$
s=2^{l_{0}} p_{1}^{l_{1}} p_{2}^{l_{2}} \ldots p_{t}^{l_{t}} q_{1}^{m_{1}} q_{2}^{m_{2}} \ldots q_{u}^{m_{u}}
$$

be the prime factorization with $p_{i} \equiv 1(\bmod 4)(1 \leq i \leq t), q_{j} \equiv 3(\bmod 4)$ $(1 \leq j \leq u)$. And let $s^{\prime}$ be the squarefree part of $s$. Then if $l_{0}>2$, we have

$$
\left(\frac{(-1)^{k} s}{m}\right)= \begin{cases}\chi_{s^{\prime}}(m), & \text { if } l_{0} \text { is even, } \sum_{i=1}^{u} m_{i} \text { is even and } k \text { is even, } \\ \chi_{-4 s^{\prime}}(m), & \text { if } l_{0} \text { is even, } \sum_{i=1}^{u} m_{i} \text { is even and } k \text { is odd, } \\ \chi_{4 s^{\prime}}(m), & \text { if } l_{0} \text { is even, } \sum_{i=1}^{u} m_{i} \text { is odd and } k \text { is even, } \\ \chi_{-s^{\prime}}(m), & \text { if } l_{0} \text { is even, } \sum_{i=1}^{u} m_{i} \text { is odd and } k \text { is odd, } \\ \chi_{4 s^{\prime}}(m), & \text { if } l_{0} \text { is odd, } \sum_{i=1}^{u} m_{i} \text { is even and } k \text { is even, } \\ \chi_{-4 s^{\prime}}(m), & \text { if } l_{0} \text { is odd, } \sum_{i=1}^{u} m_{i} \text { is even and } k \text { is odd, } \\ \chi_{4 s^{\prime}}(m), & \text { if } l_{0} \text { is odd, } \sum_{i=1}^{u} m_{i} \text { is odd and } k \text { is even, } \\ \chi_{-4 s^{\prime}}(m), & \text { if } l_{0} \text { is odd, } \sum_{i=1}^{u} m_{i} \text { is odd and } k \text { is odd. }\end{cases}
$$




\subsection{Eta Quotients in $M_{2}\left(\Gamma_{0}(16), \chi_{8}\right)$}

Previously in [72] K. S. Williams has given all eta quotients in $M_{2}\left(\Gamma_{0}(12)\right)$ along with their Fourier series expansions. Inspired by this work, in [4, 8, 5, 9, 10] we gave all eta quotients in various spaces of modular forms along with their Fourier series expansions. And in [48], Martin gave all multiplicative eta quotients. We now discover that using Theorems 4.2.4 and 4.2.6, it is always possible to give complete lists of eta quotients in any modular form space with positive integer weight. In this section we prove the following theorem, which gives the list of all eta quotients in $M_{2}\left(\Gamma_{0}(16), \chi_{8}\right)$.

Theorem 4.3.1. Let $f(z)$ be an eta quotient given by

$$
f(z)=\eta^{r_{1}}(z) \eta^{r_{2}}(2 z) \eta^{r_{4}}(4 z) \eta^{r_{8}}(8 z) \eta^{r_{16}}(16 z) .
$$

Then $f(z) \in M_{2}\left(\Gamma_{0}(16), \chi_{8}\right)$ if and only if

$$
\begin{aligned}
& v_{1}(f(z)), v_{1 / 2}(f(z)), v_{1 / 8}(f(z)) v_{1 / 16}(f(z)) \in\{0,1,2,3,4\} \\
& v_{1 / 4}(f(z)), v_{3 / 4}(f(z)) \in\left\{\frac{1}{2}, \frac{3}{2}, \frac{5}{2}, \frac{7}{2}\right\} \\
& \quad \sum_{r \in R\left(\Gamma_{0}(16)\right)} v_{r}(f(z))=4 .
\end{aligned}
$$

Note that $r_{\delta}(\delta \mid 16)$ are given by

$$
r_{1}=-2 v_{1 / 2}+2 v_{1},
$$




$$
\begin{aligned}
& r_{2}=-4 v_{1 / 4}+5 v_{1 / 2}-v_{1}, \\
& r_{4}=10 v_{1 / 4}-2 v_{1 / 2}-2 v_{1 / 8} \\
& r_{8}=-4 v_{1 / 4}-v_{5}+5 v_{1 / 8} \\
& r_{16}=2 v_{1 / 16}-2 v_{1 / 8}
\end{aligned}
$$

Proof. Let $r_{\delta} \in \mathbb{Z}$ for all $\delta \mid 16$. Let $f(z)$ be an eta quotient given by (4.3.1). By Theorem 2.2.3, we have

$$
R\left(\Gamma_{0}(16)\right)=\left\{1, \frac{1}{2}, \frac{1}{4}, \frac{3}{4}, \frac{1}{8}, \frac{1}{16}\right\} .
$$

Then by 4.1.4 we have

$$
\begin{aligned}
& \frac{2}{3} r_{1}+\frac{1}{3} r_{2}+\frac{1}{6} r_{4}+\frac{1}{12} r_{8}+\frac{1}{24} r_{16}=v_{1}(f(z)), \\
& \frac{1}{6} r_{1}+\frac{1}{3} r_{2}+\frac{1}{6} r_{4}+\frac{1}{12} r_{8}+\frac{1}{24} r_{16}=v_{1 / 2}(f(z)), \\
& \frac{1}{24} r_{1}+\frac{1}{12} r_{2}+\frac{1}{6} r_{4}+\frac{1}{12} r_{8}+\frac{1}{24} r_{16}=v_{1 / 4}(f(z)), \\
& \frac{1}{24} r_{1}+\frac{1}{12} r_{2}+\frac{1}{6} r_{4}+\frac{1}{3} r_{8}+\frac{1}{6} r_{16}=v_{1 / 8}(f(z)), \\
& \frac{1}{24} r_{1}+\frac{1}{12} r_{2}+\frac{1}{6} r_{4}+\frac{1}{3} r_{8}+\frac{2}{3} r_{16}=v_{1 / 16}(f(z))
\end{aligned}
$$

We solve the above system of linear equations for $r_{\delta}(\delta \mid N)$

$$
\begin{aligned}
& r_{1}=-2 v_{1 / 2}+2 v_{1}, \\
& r_{2}=-4 v_{1 / 4}+5 v_{1 / 2}-v_{1}, \\
& r_{4}=10 v_{1 / 4}-2 v_{1 / 2}-2 v_{1 / 8},
\end{aligned}
$$




$$
\begin{aligned}
& r_{8}=-4 v_{1 / 4}-v_{1 / 16}+5 v_{1 / 8}, \\
& r_{16}=2 v_{1 / 16}-2 v_{1 / 8} .
\end{aligned}
$$

Then by Remark 4.2.5, eta quotients in $M_{2}\left(\Gamma_{0}(16), \chi_{8}\right)$ are determined by the orders of cusps $1 / c$. Let $f(z) \in M_{2}\left(\Gamma_{0}(16), \chi_{8}\right)$. We have

$$
\chi_{8}(17)=1, \chi_{8}(9)=1, \chi_{8}(5)=-1 .
$$

Hence by Theorem 4.2 .4 we have

$$
\begin{aligned}
& \sum_{r \in R\left(\Gamma_{0}(16)\right)} v_{r}(f(z))=2 \sum_{c \mid 16} \frac{\phi\left(\operatorname{gcd}\left(c, \frac{16}{c}\right)\right)}{\operatorname{gcd}\left(c^{2}, 16\right)}=4, \\
& v_{1}(f(z)), v_{1 / 2}(f(z)), v_{1 / 8}(f(z)) v_{1 / 16}(f(z)) \in\{0,1,2,3,4\}, \\
& v_{1 / 4}(f(z)), v_{3 / 4}(f(z)) \in\left\{\frac{1}{2}, \frac{3}{2}, \frac{5}{2}, \frac{7}{2}\right\} .
\end{aligned}
$$

Conversely, let $f(z)$ satisfy 4.3.2)-4.3.4, then by Theorem 4.2.6, $f(z) \in$ $M_{2}\left(\Gamma_{0}(16), \chi\right)$, where

$$
\chi=\left(\frac{s}{*}\right)
$$

with $s=2^{\left|r_{2}\right|} 4^{\left|r_{4}\right|} 8^{\left|r_{8}\right|} 16^{\left|r_{16}\right|}=2^{\left|r_{2}\right|+2\left|r_{4}\right|+3\left|r_{8}\right|+4\left|r_{16}\right|}$. Then by 4.3.2, 4.3.3 and 4.2.13, we have $\chi=\chi_{8}$.

Subject to constraints 4.3.2 4.3.4), using MAPLE we find 48 eta quotients in $M_{2}\left(\Gamma_{0}(16), \chi_{8}\right)$. 


\subsection{Eta Quotients in $M_{2}\left(\Gamma_{0}(N)\right)$ for $N=p_{1} p_{2}$}

In this section we give complete lists of eta quotients in $M_{2}\left(\Gamma_{0}(N)\right)$, where $N$ is a multiple of two distinct primes.

Theorem 4.4.1. Let $p_{1}$ and $p_{2}$ be two distinct prime numbers, say $p_{1}<p_{2}$. Let $N=p_{1} p_{2}$. Then the list of all eta quotients in $M_{2}\left(\Gamma_{0}(N)\right)$ is given in Table 4.4.1 below.

Table 4.4.1: List of all eta quotients in $M_{2}\left(\Gamma_{0}(N)\right)$ for $N=$ $p_{1} p_{2}$

\begin{tabular}{l|l}
\hline \hline$N=p_{1} p_{2}$ & List of all eta quotients in $M_{2}\left(\Gamma_{0}(N)\right)$ \\
\hline \hline$p_{1}=2, p_{2}=3$ & $\eta_{N}[2,-4,-6,12](z), \eta_{N}[-1,-1,3,3](z)$, \\
& $\eta_{N}[-4,2,12,-6](z), \eta_{N}[-2,4,-2,4](z)$, \\
& $\eta_{N}[-5,7,7,-5](z), \eta_{N}[-6,12,2,-4](z)$, \\
& $\eta_{N}[7,-5,-5,7](z), \eta_{N}[4,-2,4,-2](z)$, \\
& $\eta_{N}[3,3,-1,-1](z), \eta_{N}[12,-6,-4,2](z)$ \\
\hline$p_{1}=2, p_{2}=7$ & $\eta_{N}[-2,4,-2,4](z), \eta_{N}[-3,5,5,-3](z)$, \\
& $\eta_{N}[1,1,1,1](z), \eta_{N}[5,-3,-3,5](z)$, \\
\hline$p_{1}=2, p_{2} \equiv 7(\bmod 24), p_{2}>7$ & $\eta_{N}[4,-2,4,-2](z)$ \\
\hline$p_{1}=2, p_{2} \equiv 11(\bmod 24)$ & $\eta_{N}[-2,4,-2,4](z), \eta_{N}[1,1,1,1](z)$, \\
& $\eta_{N}[4,-2,4,-2](z)$ \\
\hline & $\eta_{N}[-2,4,-2,4](z), \eta_{N}[0,2,0,2](z)$, \\
& $\eta_{N}[2,0,2,0](z), \eta_{N}[4,-2,4,-2](z)$ \\
\hline
\end{tabular}


Table 4.4.1 - Continued from previous page

\begin{tabular}{|c|c|}
\hline$N=p_{1} p_{2}$ & List of all eta quotients in $M_{2}\left(\Gamma_{0}(N)\right)$ \\
\hline$p_{1}=2, p_{2} \equiv 19(\bmod 24)$ & $\begin{array}{c}\eta_{N}[-2,4,-2,4](z), \eta_{N}[-1,3,3,-1](z), \\
\eta_{N}[3,-1,-1,3](z), \eta_{N}[4,-2,4,-2](z)\end{array}$ \\
\hline$p_{1}=2, p_{2} \equiv 23(\bmod 24)$ & $\begin{array}{l}\eta_{N}[-2,4,-2,4](z), \eta_{N}[-1,3,-1,3](z), \\
\eta_{N}[0,2,0,2](z), \eta_{N}[1,1,1,1](z), \\
\eta_{N}[2,0,2,0](z), \eta_{N}[3,-1,3,-1](z), \\
\eta_{N}[4,-2,4,-2](z)\end{array}$ \\
\hline$p_{1}=3, p_{2}=5$ & $\begin{array}{l}\eta_{N}[-1,3,-1,3](z), \eta_{N}[-2,4,4,-2](z), \\
\eta_{N}[1,1,1,1](z), \eta_{N}[4,-2,-2,4](z), \\
\eta_{N}[3,-1,3,-1](z)\end{array}$ \\
\hline$p_{1}=3, p_{2} \equiv 5(\bmod 12), p_{2}>5$ & $\begin{array}{l}\eta_{N}[-1,3,-1,3](z), \eta_{N}[1,1,1,1](z), \\
\eta_{N}[3,-1,3,-1](z)\end{array}$ \\
\hline$p_{1}=3, p_{2} \equiv 11(\bmod 12)$ & $\begin{array}{l}\eta_{N}[-1,3,-1,3](z), \eta_{N}[0,2,0,2](z), \\
\eta_{N}[1,1,1,1](z), \eta_{N}[2,0,2,0](z) \\
\eta_{N}[3,-1,3,-1](z)\end{array}$ \\
\hline$p_{1} \equiv 1, p_{2} \equiv 11(\bmod 12)$ & $\begin{array}{l}\eta_{N}[0,2,0,2](z), \eta_{N}[0,2,2,0](z) \\
\eta_{N}[1,1,1,1](z), \eta_{N}[2,0,0,2](z) \\
\eta_{N}[2,0,2,0](z)\end{array}$ \\
\hline $\begin{array}{c}p_{1} \equiv 5, p_{2} \equiv 7(\bmod 12), p_{2}>7 \\
\text { or } p_{1} \equiv 7, p_{2} \equiv 5(\bmod 12)\end{array}$ & $\begin{array}{l}\eta_{N}[0,2,2,0](z), \eta_{N}[1,1,1,1](z) \\
\eta_{N}[2,0,0,2](z)\end{array}$ \\
\hline $\begin{array}{l}p_{1} \equiv 5, p_{2} \equiv 11(\bmod 12) \\
\quad \text { or } p_{1} \equiv 7, p_{2} \equiv 11(\bmod 12)\end{array}$ & $\begin{array}{l}\eta_{N}[0,2,0,2](z), \eta_{N}[1,1,1,1](z) \\
\eta_{N}[2,0,2,0](z)\end{array}$ \\
\hline
\end{tabular}

Continued on next page 
Table 4.4.1 - Continued from previous page

\begin{tabular}{l|l}
\hline \hline$N=p_{1} p_{2}$ & List of all eta quotients in $M_{2}\left(\Gamma_{0}(N)\right)$ \\
\hline \hline$p_{1} \equiv 11, p_{2} \equiv 1(\bmod 12)$ & $\eta_{N}[0,0,2,2](z), \eta_{N}[0,2,2,0](z)$, \\
& $\eta_{N}[1,1,1,1](z), \eta_{N}[2,0,0,2](z)$, \\
& $\eta_{N}[2,2,0,0](z)$ \\
\hline$p_{1} \equiv 11, p_{2} \equiv 5(\bmod 12)$ & $\eta_{N}[0,0,2,2](z), \eta_{N}[1,1,1,1](z)$, \\
or $p_{1} \equiv 11, p_{2} \equiv 7(\bmod 12)$ & $\eta_{N}[2,2,0,0](z)$ \\
\hline$p_{1} \equiv 11, p_{2} \equiv 11(\bmod 12)$ & $\eta_{N}[0,0,2,2](z), \eta_{N}[0,2,0,2](z)$, \\
& $\eta_{N}[1,1,1,1](z), \eta_{N}[2,0,2,0](z)$, \\
\hline otherwise & $\eta_{N}[2,2,0,0](z)$ \\
\hline
\end{tabular}

Proof. Let $p_{1}$ and $p_{2}$ be prime numbers such that $p_{1}<p_{2}$. Let $N=p_{1} p_{2}$. By Theorem 2.2.3, we have

$$
R\left(\Gamma_{0}(N)\right)=\left\{1, \frac{1}{p_{1}}, \frac{1}{p_{2}}, \frac{1}{p_{1} p_{2}}\right\}
$$

Let $f(z)=\eta_{N}\left[r_{1}, r_{2}, r_{3}, r_{4}\right](z)$ be an eta quotient. Then we calculate

$$
\begin{aligned}
& v_{1}=v_{1}(f(z))=\frac{1}{24} p_{1} p_{2} r_{1}+\frac{1}{24} p_{2} r_{2}+\frac{1}{24} p_{1} r_{3}+\frac{1}{24} r_{4} \\
& v_{2}=v_{1 / p_{1}}(f(z))=\frac{1}{24} p_{2} r_{1}+\frac{1}{24} p_{1} p_{2} r_{2}+\frac{1}{24} r_{3}+\frac{1}{24} p_{1} r_{4} \\
& v_{3}=v_{1 / p_{2}}(f(z))=\frac{1}{24} p_{1} r_{1}+\frac{1}{24} r_{2}+\frac{1}{24} p_{1} p_{2} r_{3}+\frac{1}{24} p_{2} r_{4} \\
& v_{4}=v_{1 / p_{1} p_{2}}(f(z))=\frac{1}{24} r_{1}+\frac{1}{24} p_{1} r_{2}+\frac{1}{24} p_{2} r_{3}+\frac{1}{24} p_{1} p_{2} r_{4}
\end{aligned}
$$


We solve equations 4.4.1-4.4.4 for $r_{1}, r_{2}, r_{3}$ and $r_{4}$,

$$
\begin{aligned}
& r_{1}=\frac{24}{\left(1-p_{1}^{2}\right)\left(1-p_{2}^{2}\right)}\left(p_{1} p_{2} v_{1}-p_{2} v_{2}-p_{1} v_{3}+v_{4}\right), \\
& r_{2}=\frac{24}{\left(1-p_{1}^{2}\right)\left(1-p_{2}^{2}\right)}\left(-p_{2} v_{1}+p_{1} p_{2} v_{2}+v_{3}-p_{1} v_{4}\right), \\
& r_{3}=\frac{24}{\left(1-p_{1}^{2}\right)\left(1-p_{2}^{2}\right)}\left(-p_{1} v_{1}+v_{2}+p_{1} p_{2} v_{3}-p_{2} v_{4}\right), \\
& r_{4}=\frac{24}{\left(1-p_{1}^{2}\right)\left(1-p_{2}^{2}\right)}\left(v_{1}-p_{1} v_{2}-p_{2} v_{3}+p_{1} p_{2} v_{4}\right) .
\end{aligned}
$$

In order to prove the theorem there are three main cases we need to address:

Case 1: $p_{1}<23$ and $p_{2}<23$,

Case 2: $p_{1}<23$ and $p_{2} \geq 23$,

Case 3: $p_{1} \geq 23$ and $p_{2} \geq 23$.

We illustrate the proof of Case 1 with $p_{1}=2$ and $p_{2}=3$ as the rest can be proven similarly. Now assume that $f(z) \in M_{2}\left(\Gamma_{0}(6)\right)$. By Theorem 4.2.4. we have $\sum_{r \in R\left(\Gamma_{0}(6)\right)} v_{r}=2$ and $v_{1}, v_{2}, v_{3}, v_{4} \in \mathbb{N}_{0}$. That is, we have

$$
v_{1}, v_{2}, v_{3}, v_{4} \in\{0,1,2\} \text { and } v_{1}+v_{2}+v_{3}+v_{4}=2 .
$$

We put $p_{1}=2, p_{2}=3$ in equations 4.4.5-4.4.8,

$$
\begin{aligned}
& r_{1}=6 v_{1}-3 v_{2}-2 v_{3}+v_{4}, \\
& r_{2}=-3 v_{1}+6 v_{2}+v_{3}-2 v_{4},
\end{aligned}
$$




$$
\begin{aligned}
& r_{3}=-2 v_{1}+v_{2}+6 v_{3}-3 v_{4}, \\
& r_{4}=v_{1}-2 v_{2}-3 v_{3}+6 v_{4} .
\end{aligned}
$$

We put values of $v_{1}, v_{2}, v_{3}, v_{4}$, in 4.4 .9 -4.4.12 to get a set of all possible eta quotients in $M_{2}\left(\Gamma_{0}(6)\right)$ :

$$
\begin{aligned}
& \eta_{6}[2,-4,-6,12](z), \quad \eta_{6}[-1,-1,3,3](z), \\
& \eta_{6}[-4,2,12,-6](z), \eta_{6}[-2,4,-2,4](z), \\
& \eta_{6}[-5,7,7,-5](z), \eta_{6}[-6,12,2,-4](z), \\
& \eta_{6}[7,-5,-5,7](z), \eta_{6}[4,-2,4,-2](z), \\
& \eta_{6}[3,3,-1,-1](z), \eta_{6}[12,-6,-4,2](z) .
\end{aligned}
$$

It follows from Theorem 4.2.6 that all these eta quotients are in $M_{2}\left(\Gamma_{0}(6)\right)$.

In the proofs of Cases 2 and 3, first we use Theorem 1.0.2, to determine a set of tuples $\left(r_{1}, r_{2}, r_{3}, r_{4}\right)$, that gives a set of all possible eta quotients in $M_{2}\left(\Gamma_{0}(N)\right)$. Then we eliminate those tuples whose corresponding eta quotients have negative or non integer orders at certain cusps (see Theorem 4.2.4. Then we apply Theorem 4.2.6 to the remaining tuples to find out the tuples whose corresponding eta quotient is in $M_{2}\left(\Gamma_{0}(N)\right)$.

Let us now illustrate Case 2 with $p_{1}=2$ and $p_{2} \equiv 7(\bmod 24), 7<p_{2}$. 
Let $N=p_{1} p_{2}$. Suppose $f(z) \in M_{2}\left(\Gamma_{0}(N)\right)$. Then by Theorem 1.0.2, we have

$$
\sum_{i=1}^{4}\left|r_{i}\right| \leq 2\left(\frac{24 l+8}{4 l+1}\right)<13 \text { and } \sum_{i=1}^{4} r_{i}=4
$$

There are 871 tuples of $\left(r_{1}, r_{2}, r_{3}, r_{4}\right)$ satisfying these two conditions. Let $p_{1}=2$ and $p_{2}=24 l+7$ in 4.4.1 4.4 .4 , then

$$
\begin{aligned}
& v_{1}=l\left(2 r_{1}+r_{2}\right)+\frac{7\left(2 r_{1}+r_{2}\right)+\left(2 r_{3}+r_{4}\right)}{24}, \\
& v_{2}=l\left(r_{1}+2 r_{2}\right)+\frac{7\left(r_{1}+2 r_{2}\right)+\left(r_{3}+2 r_{4}\right)}{24}, \\
& v_{3}=l\left(2 r_{3}+r_{4}\right)+\frac{\left(2 r_{1}+r_{2}\right)+7\left(2 r_{3}+r_{4}\right)}{24}, \\
& v_{4}=l\left(r_{3}+2 r_{4}\right)+\frac{\left(r_{1}+2 r_{2}\right)+7\left(r_{3}+2 r_{4}\right)}{24} .
\end{aligned}
$$

For the tuples determined by Theorem 1.0.2, we have $2 r_{i}+r_{j} \leq 16$, for all $1 \leq i, j \leq 4$. Then we have

$$
\begin{aligned}
& \text { if } 2 r_{1}+r_{2}<0 \text {, then } v_{1}<0 \text {, } \\
& \text { if } r_{1}+2 r_{2}<0 \text {, then } v_{2}<0 \text {, } \\
& \text { if } 2 r_{3}+r_{4}<0 \text {, then } v_{3}<0 \text {, } \\
& \text { if } r_{3}+2 r_{4}<0 \text {, then } v_{4}<0 \text {. }
\end{aligned}
$$

That is, the eta quotients corresponding to the tuples that satisfy 4.4.13, $4.4 .14,4.4 .15$ or 4.4.16 are not holomorphic at the cusp $1, \frac{1}{p_{1}}, \frac{1}{p_{2}}$ or 
$\frac{1}{p_{1} p_{2}}$, respectively, and cannot be in $M_{2}\left(\Gamma_{0}(N)\right.$. Eliminating those tuples we are left with only 155 out of 871 . That is all eta quotients in $M_{2}\left(\Gamma_{0}(N)\right.$ are among these 155 tuples. Of these tuples only for

$$
\begin{aligned}
\left(r_{1}, r_{2}, r_{3}, r_{4}\right)= & (-4,8,0,0),(-3,6,-1,2),(-2,4,-2,4), \\
& (-1,2,-3,6),(0,0,-4,8),(0,3,2,-1), \\
& (1,1,1,1),(2,-1,0,3),(4,-2,4,-2),
\end{aligned}
$$

we have $v_{1} \in \mathbb{N}_{0}$. We apply Theorem 4.2.6 to the tuples given in 4.4.17) to see only for

$$
\left(r_{1}, r_{2}, r_{3}, r_{4}\right)=(-2,4,-2,4),(1,1,1,1),(4,-2,4,-2),
$$

the corresponding $f(z)$ are modular forms in $M_{2}\left(\Gamma_{0}(N)\right)$.

Let us now tackle Case 3. Let $p_{1}, p_{2} \geq 23$. Let $N=p_{1} p_{2}$. Suppose $f(z) \in M_{2}\left(\Gamma_{0}(N)\right)$. Then by Theorem 1.0 .2 we have

$$
\sum_{i=1}^{4}\left|r_{i}\right| \leq 2\left(\frac{p_{1}+1}{p_{1}-1}\right)\left(\frac{p_{2}+1}{p_{2}-1}\right)<5 \text { and } \sum_{i=1}^{4} r_{i}=4
$$

There are 35 such tuples of $\left(r_{1}, r_{2}, r_{3}, r_{4}\right)$. Additionally, if

$$
r_{2}+r_{4} \not \equiv 0 \quad(\bmod 2) \text { or } r_{3}+r_{4} \not \equiv 0 \quad(\bmod 2)
$$

then $f(z) \notin M_{2}\left(\Gamma_{0}(N)\right)$. Leaving us only 11 possible tuples. Let $p_{1}=12 l_{1}+t_{1}$ 
and $p_{2}=12 l_{2}+t_{2}$, with $l_{1}, l_{2} \geq 1$. Then we have

$$
\begin{aligned}
v_{1}= & r_{1}\left(6 l_{1} l_{2}+\frac{1}{2} l_{1} t_{2}+\frac{1}{2} t_{1} l_{2}+\frac{1}{24} t_{1} t_{2}\right)+r_{2}\left(\frac{1}{2} l_{2}+\frac{1}{24} t_{2}\right) \\
& +r_{3}\left(\frac{1}{2} l_{1}+\frac{1}{24} t_{1}\right)+r_{4} \frac{1}{24} \\
= & r_{1}\left(6 l_{1} l_{2}+\frac{1}{2} l_{1} t_{2}+\frac{1}{2} t_{1} l_{2}\right)+r_{2}\left(\frac{1}{2} l_{2}\right)+r_{3}\left(\frac{1}{2} l_{1}\right) \\
& +\left(r_{1} \frac{1}{24} t_{1} t_{2}+r_{2} \frac{1}{24} t_{2}+r_{3} \frac{1}{24} t_{1}+r_{4} \frac{1}{24}\right) .
\end{aligned}
$$

By examining the above equation of $v_{1}$ for the remaining 11 tuples of $\left(r_{1}, r_{2}, r_{3}, r_{4}\right)$ we conclude that if

$$
\left(t_{1}, t_{2}\right)=(1,1),(1,5),(1,7),(5,1),(5,5),(7,1),(7,7)
$$

then $v_{1} \notin \mathbb{N}_{0}$. Then by Theorem 4.2.6, there no eta quotients in $M_{2}\left(\Gamma_{0}(N)\right)$ if

$$
p_{1} \equiv t_{1} \quad(\bmod 12) \text { and } p_{2} \equiv t_{2} \quad(\bmod 12)
$$

where $\left(t_{1}, t_{2}\right)$ is given in 4.4.18.

We continue the proof of Case 3 with $p_{1} \equiv 11(\bmod 12)$ and $p_{2} \equiv 11$ $(\bmod 12)$. Let $p_{1}=12 l_{1}+11$ and $p_{2}=12 l_{2}+11$, with $l_{1}, l_{2} \geq 1$. Let $N=p_{1} p_{2}$. Suppose $f(z) \in M_{2}\left(\Gamma_{0}(N)\right)$. We have

$$
v_{1}=r_{1}\left(6 l_{1} l_{2}+\frac{1}{2} l_{1} 11+\frac{1}{2} 11 l_{2}+\frac{1}{24} 121\right)+r_{2}\left(\frac{1}{2} l_{2}+\frac{1}{24} 11\right)
$$




$$
\begin{aligned}
& +r_{3}\left(\frac{1}{2} l_{1}+\frac{1}{24} 11\right)+r_{4} \frac{1}{24} \\
= & r_{1}\left(6 l_{1} l_{2}+\frac{1}{2} l_{1} 11+\frac{1}{2} 11 l_{2}\right)+r_{2}\left(\frac{1}{2} l_{2}\right)+r_{3}\left(\frac{1}{2} l_{1}\right) \\
& +\left(r_{1} \frac{121}{24}+r_{2} \frac{11}{24}+r_{3} \frac{11}{24}+r_{4} \frac{1}{24}\right) .
\end{aligned}
$$

Examining $v_{1}$ for 11 tuples of $\left(r_{1}, r_{2}, r_{3}, r_{4}\right)$, we conclude, $v_{1} \in \mathbb{N}_{0}$ only if

$$
\left(r_{1}, r_{2}, r_{3}, r_{4}\right)=(0,0,2,2),(0,2,0,2),(1,1,1,1),(2,0,2,0),(2,2,0,0) .
$$

We apply Theorem 4.2.6 to see that the eta quotients corresponding to above tuples are indeed in $M_{2}\left(\Gamma_{0}(N)\right)$.

\subsection{Number of Eta Quotients in $M_{2}\left(\Gamma_{0}(N)\right)$ for

$$
N \leq 100
$$

Using arguments similar to previous section we find all eta quotients in $M_{2}\left(\Gamma_{0}(N)\right)$ for all $N \leq 100$. We give the numbers in two different figures. In Figure 4.5.1. we include spaces with less than 500 eta quotients. In Figure 4.5.2 we include the ones with more than 500 eta quotients. The figures suggest that the number of eta quotients in a space is related to the number of divisors of $N$ and the difference between consecutive divisors of $N$. Please note that for presentation purposes we needed to limit the Figure 4.5.2 at 25000. The only level with more than 25000 eta quotients is $N=72$ with 


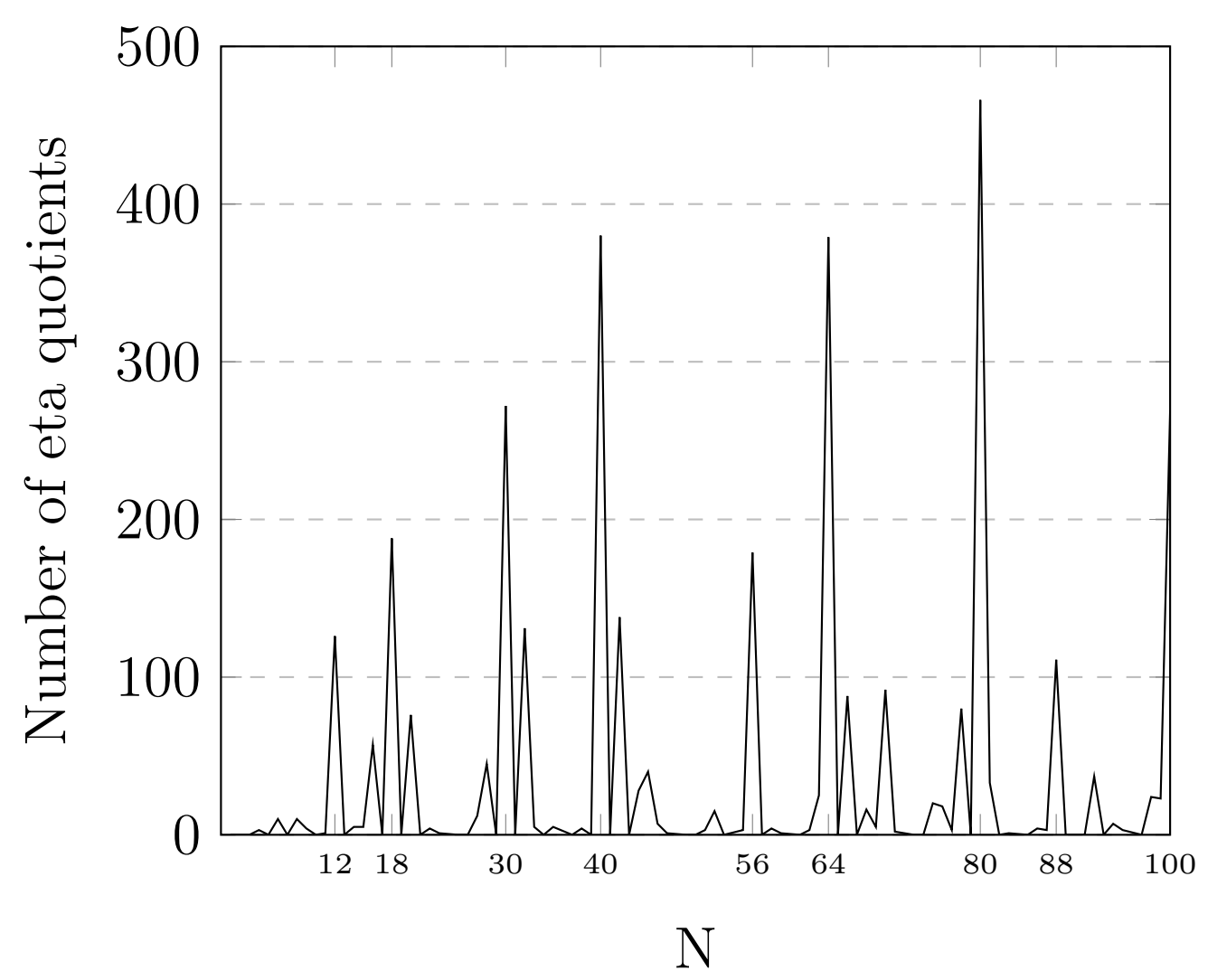

Figure 4.5.1: Number of eta quotients in $M_{2}\left(\Gamma_{0}(N)\right)$

45796 eta quotients.

Note that $M_{k}\left(\Gamma_{0}(N), \chi\right) \subseteq M_{k}\left(\Gamma_{0}(t N), \chi\right)$ for all $t \in \mathbb{N}$. So the number of eta quotients in $M_{k}\left(\Gamma_{0}(t N), \chi\right)$ is always greater than or equal to the number of eta quotients in $M_{k}\left(\Gamma_{0}(N), \chi\right)$. In some cases the generating function that we are interested in might fall in a space where there are not enough eta quotients to express the generating function explicitly. In these cases (in Chapter 8) we study on a larger level which has more eta quotients. See [35] for a similar idea. 


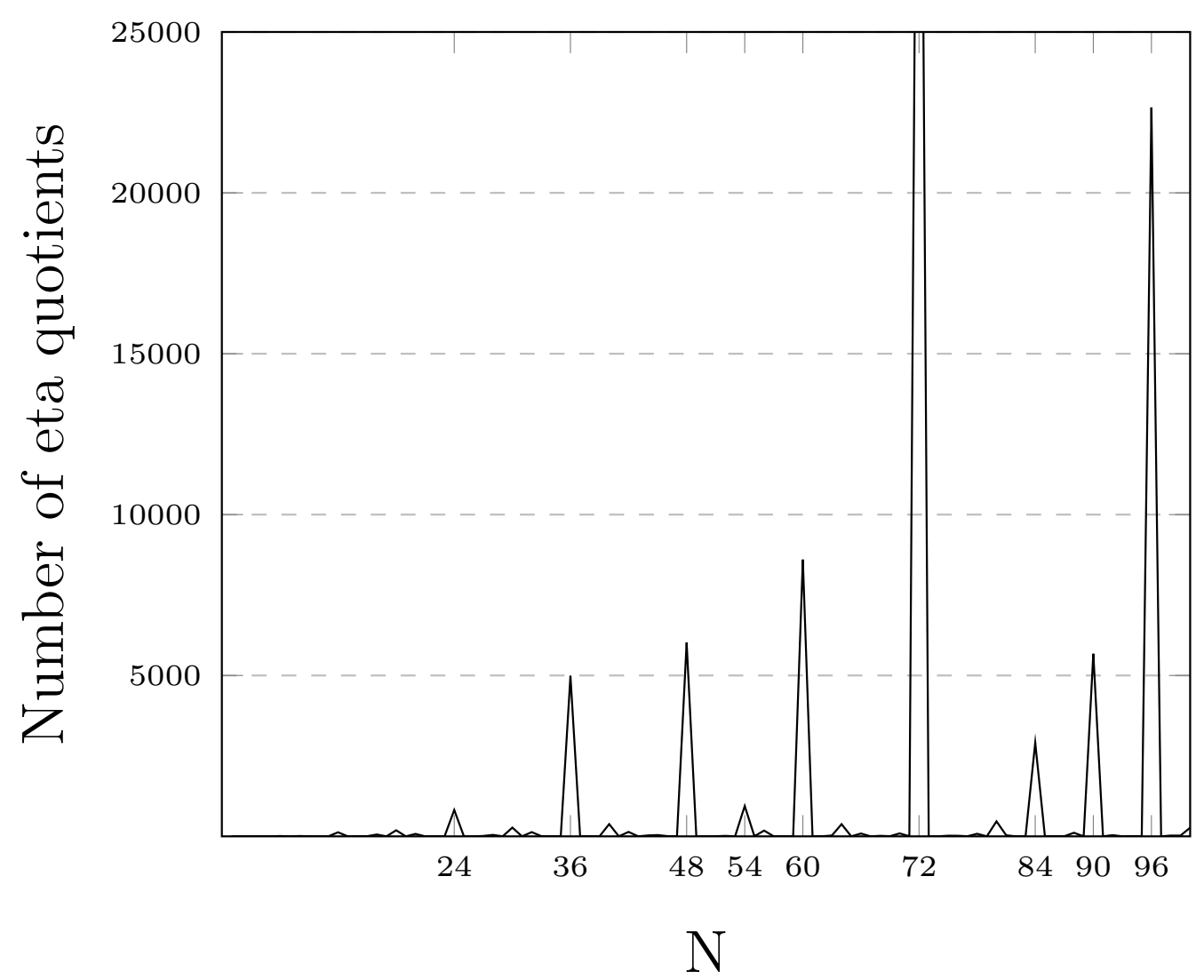

Figure 4.5.2: Number of eta quotients in $M_{2}\left(\Gamma_{0}(N)\right)$ 


\section{Part II}

\section{Main Research}




\section{Chapter 5}

\section{Bases for $S_{k}\left(\Gamma_{0}(12), \chi\right)$ for $\chi=\chi_{1}, \chi_{-3}, \chi_{-4}, \chi_{12}$}

In [58], after the proof that $M_{2 k}\left(\Gamma_{0}(1)\right)$ is generated by eta quotients for all $k \geq 1$, Ono raises the following question concerning eta quotients.

[58, Problem 1.68]: Classify the spaces of modular forms which are generated by eta-quotients.

Let $N \in \mathbb{N}$. In 2015, Rouse and Webb proved that if $M_{2}\left(\Gamma_{0}(N)\right)$ is generated by eta quotients then, for all $k>1, M_{2 k}\left(\Gamma_{0}(N)\right)$ can be generated by eta quotients [65]. But no explicit basis that works for all $k \geq 1$ was provided.

In [57, p. 253], it is argued that the cusp part of a modular form rarely has a simple description. In this chapter, in order to give a description for the cusp part of modular forms in $M_{k}\left(\Gamma_{0}(12), \chi\right)$ using eta quotients, we construct bases for $S_{k}\left(\Gamma_{0}(12), \chi\right)$ for all $k \geq 1$ and $\chi$ a real character with conductor dividing 12 in terms of eta quotients. The families of eta quotients in this chapter are new. In Appendix $\mathrm{A}$ we give further new examples of such families of eta quotients. One can produce such other families of eta quotients 
after careful investigation of orders of eta quotients at cusps. Results from 65] support this idea.

\subsection{New Families of Eta Quotients and Bases for $S_{k}\left(\Gamma_{0}(12), \chi\right)$}

Let $k \in \mathbb{N}$. In this section we will give bases for $S_{k}\left(\Gamma_{0}(12), \chi\right)$ for all $k \geq 1$, and for $\chi=\chi_{1}, \chi_{-3}, \chi_{-4}, \chi_{12}$. To find a basis for the Eisenstein subspaces is straightforward application of Theorem 3.2.2. As discussed above it is usually challenging to give a simple description for the cusp subspaces, [57, p. 253]. For this purpose we define two families of eta quotients by

$$
\begin{aligned}
C_{j, k}(z) & =\frac{\eta^{6 k-3 j-15}(q) \eta^{k-2 j+2}\left(q^{6}\right) \eta^{3 j-3}\left(q^{12}\right)}{\eta^{3 k-2 j-10}\left(q^{2}\right) \eta^{2 k-j-5}\left(q^{3}\right) \eta^{j-1}\left(q^{4}\right)} \\
D_{j, k}(z) & =\frac{\eta^{6 k-3 j-11}(q) \eta^{k-2 j}\left(q^{6}\right) \eta^{3 j-2}\left(q^{12}\right)}{\eta^{3 k-2 j-6}\left(q^{2}\right) \eta^{2 k-j-5}\left(q^{3}\right) \eta^{j-2}\left(q^{4}\right)}
\end{aligned}
$$

Lemma 5.1.1. For all $j \in \mathbb{Z}$, the Fourier series expansions of $C_{j, k}(q)$ and $D_{j, k}(q)$ are of the following forms

$$
\begin{gathered}
C_{j, k}(z)=q^{j}+\sum_{n>j} c_{n} q^{n}, \\
D_{j, k}(z)=q^{j}+\sum_{n>j} d_{n} q^{n}
\end{gathered}
$$

for some integers $c_{n}$ and $d_{n}$. 
Proof. Using (4.1.4), we obtain

$$
v_{\infty}\left(C_{j, k}(z)\right)=j \text { and } v_{\infty}\left(D_{j, k}(z)\right)=j
$$

Hence the result follows.

As in Lemma 5.1.1, the eta quotients in the bases we are giving in this thesis have different orders at $\infty$. Then the linear independence follows without further effort. This setting will also be helpful in numerical calculations. This idea is similar to the idea of 'Miller Basis', see [70, Section 2.3].

Lemma 5.1.2. Let $j \in \mathbb{N}$. If $1 \leq j \leq 2 k-5$ then

$$
C_{j, k}(z) \in \begin{cases}S_{k}\left(\Gamma_{0}(12), \chi_{1}\right) & \text { if } k>2 \text { is even } \\ S_{k}\left(\Gamma_{0}(12), \chi_{-3}\right) & \text { if } k \text { is odd } .\end{cases}
$$

If $1 \leq j \leq 2 k-4$ then

$$
D_{j, k}(z) \in \begin{cases}S_{k}\left(\Gamma_{0}(12), \chi_{-4}\right) & \text { if } k \text { is odd } \\ S_{k}\left(\Gamma_{0}(12), \chi_{12}\right) & \text { if } k>2 \text { is even } .\end{cases}
$$

Proof. Follows from Theorem 4.2.6 and 4.2.13.

Theorem 5.1.3. Bases for all nontrivial spaces $S_{k}\left(\Gamma_{0}(12), \chi\right)$ for $\chi=\chi_{1}, \chi_{-3}, \chi_{-4}, \chi_{12}$ are given in Table 5.1.1. 
Table 5.1.1: Bases for $S_{k}\left(\Gamma_{0}(12), \chi\right)$

\begin{tabular}{|l|l|l|}
\hline \hline Weight $k$ & Space & Basis \\
\hline$k \geq 4$, even & $S_{k}\left(\Gamma_{0}(12), \chi_{1}\right)$ & $\left\{C_{j, k}(z)\right\}_{1 \leq j \leq 2 k-5}$ \\
\hline$k \geq 3$, odd & $S_{k}\left(\Gamma_{0}(12), \chi_{-3}\right)$ & $\left\{C_{j, k}(z)\right\}_{1 \leq j \leq 2 k-5}$ \\
\hline$k \geq 3$, odd & $S_{k}\left(\Gamma_{0}(12), \chi_{-4}\right)$ & $\left\{D_{j, k}(z)\right\}_{1 \leq j \leq 2 k-4}$ \\
\hline$k \geq 4$, even & $S_{k}\left(\Gamma_{0}(12), \chi_{12}\right)$ & $\left\{D_{j, k}(z)\right\}_{1 \leq j \leq 2 k-4}$ \\
\hline
\end{tabular}

Proof. We deduce from the formulae in [70, Section 6.3, p. 98] that

$$
\begin{aligned}
& \operatorname{dim}\left(S_{k}\left(\Gamma_{0}(12), \chi_{1}\right)\right)= \begin{cases}0 & \text { if } k \text { is odd }, \\
2 k-5 & \text { if } k>2 \text { is even; }\end{cases} \\
& \operatorname{dim}\left(S_{k}\left(\Gamma_{0}(12), \chi_{-3}\right)\right)= \begin{cases}2 k-5 & \text { if } k \text { is odd }, \\
0 & \text { if } k \text { is even; }\end{cases} \\
& \operatorname{dim}\left(S_{k}\left(\Gamma_{0}(12), \chi_{-4}\right)\right)= \begin{cases}2 k-4 & \text { if } k \text { is odd }, \\
0 & \text { if } k \text { is even; }\end{cases} \\
& \operatorname{dim}\left(S_{k}\left(\Gamma_{0}(12), \chi_{12}\right)\right)= \begin{cases}0 & \text { if } k \text { is odd }, \\
2 k-4 & \text { if } k>2 \text { is even. }\end{cases}
\end{aligned}
$$

Hence the assertions follow from Lemmas 5.1.1 and 5.1.2,

In Appendix A, we define other families of eta quotients to give bases 
for $S_{2 k}\left(\Gamma_{0}(20)\right)$ and $S_{2 k}\left(\Gamma_{0}(28)\right)$ for all $k \in \mathbb{N}$. In [65], it has been proven that if $M_{2}\left(\Gamma_{0}(N)\right)$ is generated by eta quotients then $M_{2 k}\left(\Gamma_{0}(N)\right)$ is also generated by eta quotients for all $k>1$. Our numerical studies indicate that, using the eta quotients in $M_{k}\left(\Gamma_{0}(N)\right)$, one can generate eta quotients in spaces $M_{k^{\prime}}\left(\Gamma_{0}(N)\right)$ with $k^{\prime}>k$. This process may produce bases for larger weight spaces using the bases of smaller weight spaces. Our observations suggest that such bases exist whenever the divisors of $N$ are not 'sparsely distributed'. 


\section{Chapter 6}

\section{Fourier Series Expansions of Some Modular Forms at Certain Cusps}

In this chapter, we find Fourier series expansions of some modular forms at certain cusps. In the first section we use the formula given by 4.1.3 to compute Fourier series expansions of the eta quotients $\varphi(z), \varphi(3 z)$ defined by (1.0.4) at relevant cusps of $\Gamma_{0}(12)$. In the second section, we find the Fourier series expansion of the Eisenstein series $E_{2 k}(t z),(t \in \mathbb{N})$ at cusps $\frac{1}{c}$. We will need the results of these sections in Chapter 7 , where we prove an extension of the Ramanujan-Mordell formula.

\subsection{Fourier Series Expansions of $\varphi(z)$ and $\varphi(3 z)$ at Certain Cusps}

In this section, we apply Theorem 4.1.1 with given matrices to find Fourier series expansions of Dedekind eta functions $\eta(t z)(t \mid 12)$ at the cusps $1, \frac{1}{3}, \frac{1}{4}$. 
For $c \in \mathbb{Z}$ we define $A_{c}$ as

$$
A_{c}=\left(\begin{array}{cc}
-1 & 0 \\
c & -1
\end{array}\right) \in S L_{2}(\mathbb{Z})
$$

In Theorem 4.1 .1 we take $L=\left(\begin{array}{ll}x & y \\ u & v\end{array}\right)=L_{c}$ as

$$
\begin{aligned}
& L_{1}=\left(\begin{array}{ll}
-1 & 1 \\
-1 & 0
\end{array}\right), L_{2}=\left(\begin{array}{ll}
-2 & 1 \\
-1 & 0
\end{array}\right), L_{3}=\left(\begin{array}{ll}
-3 & 1 \\
-1 & 0
\end{array}\right), \\
& L_{4}=\left(\begin{array}{ll}
-4 & 1 \\
-1 & 0
\end{array}\right), L_{6}=\left(\begin{array}{ll}
-6 & 1 \\
-1 & 0
\end{array}\right), L_{12}=\left(\begin{array}{cc}
-12 & 1 \\
-1 & 0
\end{array}\right),
\end{aligned}
$$

and $A=\left(\begin{array}{ll}a & b \\ c & d\end{array}\right)=A_{1}$, where $A_{1}$ is given by 6.1.1. We obtain the Fourier series expansions of $\eta_{r}(z)$ for $r=1,2,3,4,6,12$ at the cusp 1 as

$$
\begin{aligned}
& \eta_{1}\left(A_{1}^{-1} z\right)=e^{\pi i / 3}(-z-1)^{1 / 2} \sum_{n \geq 1}\left(\frac{12}{n}\right) e\left(\frac{n^{2}}{24}(z+1)\right), \\
& \eta_{2}\left(A_{1}^{-1} z\right)=\frac{e^{5 \pi i / 12}}{2^{1 / 2}}(-z-1)^{1 / 2} \sum_{n \geq 1}\left(\frac{12}{n}\right) e\left(\frac{n^{2}}{48}(z+1)\right), \\
& \eta_{3}\left(A_{1}^{-1} z\right)=\frac{e^{\pi i / 2}}{3^{1 / 2}}(-z-1)^{1 / 2} \sum_{n \geq 1}\left(\frac{12}{n}\right) e\left(\frac{n^{2}}{72}(z+1)\right), \\
& \eta_{4}\left(A_{1}^{-1} z\right)=\frac{e^{7 \pi i / 12}}{2}(-z-1)^{1 / 2} \sum_{n \geq 1}\left(\frac{12}{n}\right) e\left(\frac{n^{2}}{96}(z+1)\right), \\
& \eta_{6}\left(A_{1}^{-1} z\right)=\frac{e^{3 \pi i / 4}}{6^{1 / 2}}(-z-1)^{1 / 2} \sum_{n \geq 1}\left(\frac{12}{n}\right) e\left(\frac{n^{2}}{144}(z+1)\right), \\
& \eta_{12}\left(A_{1}^{-1} z\right)=\frac{e^{5 \pi i / 4}}{12^{1 / 2}}(-z-1)^{1 / 2} \sum_{n \geq 1}\left(\frac{12}{n}\right) e\left(\frac{n^{2}}{288}(z+1)\right) .
\end{aligned}
$$

From 1.0 .4 and $6.1 .2-6.1 .7$ we obtain the Fourier series expansions of 
$\varphi(z)$ and $\varphi(3 z)$ at the cusp 1 as

$$
\begin{aligned}
& \varphi\left(A_{1}^{-1} z\right)=\frac{\eta_{2}^{5}\left(A_{1}^{-1} z\right)}{\eta_{1}^{2}\left(A_{1}^{-1} z\right) \eta_{4}^{2}\left(A_{1}^{-1} z\right)} \\
&=\frac{\frac{e^{\pi i / 4}}{2^{1 / 2}}(-z-1)^{1 / 2}\left(\sum_{n \geq 1}\left(\frac{12}{n}\right) e\left(\frac{n^{2}}{48}(z+1)\right)\right)^{5}}{\left(\sum_{n \geq 1}\left(\frac{12}{n}\right) e\left(\frac{n^{2}}{24}(z+1)\right)\right)^{2}\left(\sum_{n \geq 1}\left(\frac{12}{n}\right) e\left(\frac{n^{2}}{96}(z+1)\right)\right)^{2}}, \\
& \varphi\left(3 A_{1}^{-1} z\right)=\frac{\eta_{6}^{5}\left(A_{1}^{-1} z\right)}{\eta_{3}^{2}\left(A_{1}^{-1} z\right) \eta_{12}^{2}\left(A_{1}^{-1} z\right)} \\
&=\frac{e^{\pi i / 4}}{6^{1 / 2}}(-z-1)^{1 / 2}\left(\sum_{n \geq 1}\left(\frac{12}{n}\right) e\left(\frac{n^{2}}{144}(z+1)\right)\right)^{5} \\
&\left(\sum_{n \geq 1}\left(\frac{12}{n}\right) e\left(\frac{n^{2}}{72}(z+1)\right)\right)^{2}\left(\sum_{n \geq 1}\left(\frac{12}{n}\right) e\left(\frac{n^{2}}{288}(z+1)\right)\right)^{2}
\end{aligned}
$$

Similarly, by taking $A=A_{3}$ in Theorem 4.1.1 and $L$ as

$$
\begin{aligned}
& L_{1}=\left(\begin{array}{ll}
-1 & -5 \\
-3 & -16
\end{array}\right), L_{2}=\left(\begin{array}{ll}
-2 & -5 \\
-3 & -8
\end{array}\right), L_{3}=\left(\begin{array}{ll}
-1 & 1 \\
-1 & 0
\end{array}\right), \\
& L_{4}=\left(\begin{array}{ll}
-4 & -5 \\
-3 & -4
\end{array}\right), L_{6}=\left(\begin{array}{ll}
-2 & 1 \\
-1 & 0
\end{array}\right), L_{12}=\left(\begin{array}{ll}
-4 & 1 \\
-1 & 0
\end{array}\right)
\end{aligned}
$$

we obtain the Fourier series expansions of $\eta_{r}(z)$ for $r=1,2,3,4,6,12$ at the cusp $1 / 3$ as

$$
\begin{aligned}
& \eta_{1}\left(A_{3}^{-1} z\right)=e^{5 \pi i / 3}(-3 z-1)^{1 / 2} \sum_{n \geq 1}\left(\frac{12}{n}\right) e\left(\frac{n^{2}}{24}(z-5)\right) \\
& \eta_{2}\left(A_{3}^{-1} z\right)=\frac{e^{7 \pi i / 12}}{2^{1 / 2}}(-3 z-1)^{1 / 2} \sum_{n \geq 1}\left(\frac{12}{n}\right) e\left(\frac{n^{2}}{48}(z-5)\right)
\end{aligned}
$$




$$
\begin{aligned}
& \eta_{3}\left(A_{3}^{-1} z\right)=e^{\pi i / 3}(-3 z-1)^{1 / 2} \sum_{n \geq 1}\left(\frac{12}{n}\right) \exp \left(\frac{n^{2}}{24}(3 z+1)\right) \\
& \eta_{4}\left(A_{3}^{-1} z\right)=\frac{e^{17 \pi i / 12}}{2}(-3 z-1)^{1 / 2} \sum_{n \geq 1}\left(\frac{12}{n}\right) e\left(\frac{n^{2}}{96}(z-5)\right) \\
& \eta_{6}\left(A_{3}^{-1} z\right)=\frac{e^{5 \pi i / 12}}{2^{1 / 2}}(-3 z-1)^{1 / 2} \sum_{n \geq 1}\left(\frac{12}{n}\right) e\left(\frac{n^{2}}{48}(3 z+1)\right) \\
& \eta_{12}\left(A_{3}^{-1} z\right)=\frac{e^{7 \pi i / 12}}{2}(-3 z-1)^{1 / 2} \sum_{n \geq 1}\left(\frac{12}{n}\right) e\left(\frac{n^{2}}{96}(3 z+1)\right)
\end{aligned}
$$

From 1.0 .4 and $6.1 .10-6.1 .15)$ we obtain the Fourier series expansions of $\varphi(z)$ and $\varphi(3 z)$ at the cusp $1 / 3$ as

$$
\begin{aligned}
& \varphi\left(A_{3}^{-1} z\right)=\frac{\eta_{2}^{5}\left(A_{3}^{-1} z\right)}{\eta_{1}^{2}\left(A_{3}^{-1} z\right) \eta_{4}^{2}\left(A_{3}^{-1} z\right)} \\
&=\frac{\frac{e^{3 \pi i / 4}}{2^{1 / 2}}(-3 z-1)^{1 / 2}\left(\sum_{n \geq 1}\left(\frac{12}{n}\right) e\left(\frac{n^{2}}{48}(z-5)\right)\right)^{5}}{\left(\sum_{n \geq 1}\left(\frac{12}{n}\right) e\left(\frac{n^{2}}{24}(z-5)\right)\right)^{2}\left(\sum_{n \geq 1}\left(\frac{12}{n}\right) e\left(\frac{n^{2}}{96}(z-5)\right)\right)^{2}} \\
& \varphi\left(3 A_{3}^{-1} z\right)=\frac{\eta_{6}^{5}\left(A_{3}^{-1} z\right)}{\eta_{3}^{2}\left(A_{3}^{-1} z\right) \eta_{12}^{2}\left(A_{3}^{-1} z\right)} \\
&=\frac{e^{\pi i / 4}}{2^{1 / 2}}(-3 z-1)^{1 / 2}\left(\sum_{n \geq 1}\left(\frac{12}{n}\right) e\left(\frac{n^{2}}{48}(3 z+1)\right)\right)^{5} \\
&\left(\sum_{n \geq 1}\left(\frac{12}{n}\right) e\left(\frac{n^{2}}{24}(3 z+1)\right)\right)^{2}\left(\sum_{n \geq 1}\left(\frac{12}{n}\right) e\left(\frac{n^{2}}{96}(3 z+1)\right)\right)^{2}
\end{aligned}
$$


Again by taking $A=A_{4}$ in Theorem 4.1.1 and $L$ as

$$
\begin{aligned}
& L_{1}=\left(\begin{array}{ll}
-1 & 1 \\
-4 & 3
\end{array}\right), L_{2}=\left(\begin{array}{ll}
-1 & 2 \\
-2 & 3
\end{array}\right), L_{3}=\left(\begin{array}{ll}
-3 & 1 \\
-4 & 1
\end{array}\right) \\
& L_{4}=\left(\begin{array}{ll}
-1 & 4 \\
-1 & 3
\end{array}\right), L_{6}=\left(\begin{array}{ll}
-3 & 2 \\
-2 & 1
\end{array}\right), L_{12}=\left(\begin{array}{ll}
-3 & 4 \\
-1 & 1
\end{array}\right)
\end{aligned}
$$

we obtain the Fourier series expansions of $\eta_{r}(z)$ for $r=1,2,3,4,6,12$ at the cusp $1 / 4$ as

$$
\begin{aligned}
& \eta_{1}\left(A_{4}^{-1} z\right)=e^{13 \pi i / 12}(-4 z-1)^{1 / 2} \sum_{n \geq 1}\left(\frac{12}{n}\right) e\left(\frac{n^{2}}{24}(z+1)\right) \\
& \eta_{2}\left(A_{4}^{-1} z\right)=e^{\pi i / 6}(-4 z-1)^{1 / 2} \sum_{n \geq 1}\left(\frac{12}{n}\right) e\left(\frac{n^{2}}{12}(z+1)\right) \\
& \eta_{3}\left(A_{4}^{-1} z\right)=\frac{e^{5 \pi i / 12}}{3^{1 / 2}}(-4 z-1)^{1 / 2} \sum_{n \geq 1}\left(\frac{12}{n}\right) e\left(\frac{n^{2}}{72}(z+1)\right) \\
& \eta_{4}\left(A_{4}^{-1} z\right)=e^{\pi i / 12}(-4 z-1)^{1 / 2} \sum_{n \geq 1}\left(\frac{12}{n}\right) e\left(\frac{n^{2}}{6}(z+1)\right) \\
& \eta_{6}\left(A_{4}^{-1} z\right)=\frac{e^{\pi i / 3}}{3^{1 / 2}}(-4 z-1)^{1 / 2} \sum_{n \geq 1}\left(\frac{12}{n}\right) e\left(\frac{n^{2}}{36}(z+1)\right) \\
& \eta_{12}\left(A_{4}^{-1} z\right)=\frac{e^{5 \pi i / 12}}{3^{1 / 2}}(-4 z-1)^{1 / 2} \sum_{n \geq 1}\left(\frac{12}{n}\right) e\left(\frac{n^{2}}{18}(z+1)\right)
\end{aligned}
$$

From (1.0.4) and (6.1.18)-6.1.23) we obtain the Fourier series expansions of $\varphi(z)$ and $\varphi(3 z)$ at the cusp $1 / 4$ as

$$
\varphi\left(A_{4}^{-1} z\right)=\frac{\eta_{2}^{5}\left(A_{4}^{-1} z\right)}{\eta_{1}^{2}\left(A_{4}^{-1} z\right) \eta_{4}^{2}\left(A_{4}^{-1} z\right)}
$$




$$
=\frac{e^{\pi i / 2}(-4 z-1)^{1 / 2}\left(\sum_{n \geq 1}\left(\frac{12}{n}\right) e\left(\frac{n^{2}}{12}(z+1)\right)\right)^{5}}{\left(\sum_{n \geq 1}\left(\frac{12}{n}\right) e\left(\frac{n^{2}}{24}(z+1)\right)\right)^{2}\left(\sum_{n \geq 1}\left(\frac{12}{n}\right) e\left(\frac{n^{2}}{6}(z+1)\right)\right)^{2}},
$$

$$
\begin{aligned}
\varphi\left(3 A_{4}^{-1} z\right) & =\frac{\eta_{6}^{5}\left(A_{4}^{-1} z\right)}{\eta_{3}^{2}\left(A_{4}^{-1} z\right) \eta_{12}^{2}\left(A_{4}^{-1} z\right)} \\
& =\frac{\frac{1}{3}(-4 z-1)^{1 / 2}\left(\sum_{n \geq 1}\left(\frac{12}{n}\right) e\left(\frac{n^{2}}{36}(z+1)\right)\right)^{5}}{\left(\sum_{n \geq 1}\left(\frac{12}{n}\right) e\left(\frac{n^{2}}{72}(z+1)\right)\right)^{2}\left(\sum_{n \geq 1}\left(\frac{12}{n}\right) e\left(\frac{n^{2}}{18}(z+1)\right)\right)^{2}} .
\end{aligned}
$$

For $k \geq 2$ an integer and $i$ an integer with $0 \leq i \leq 2 k$ we give the first terms of the Fourier series expansions of $\varphi^{4 k-2 i}(z) \varphi^{2 i}(3 z)$ at cusps $\infty, 1, \frac{1}{2}, \frac{1}{3}, \frac{1}{4}, \frac{1}{6}$ in the following table. We deduce them (except for the cusps $1 / 2$ and $1 / 6$ ) from 6.1.8, 6.1.9), 6.1.16, 6.1.17, 6.1.24 and 6.1.25).

Table 6.1.1: First terms of $\varphi^{4 k-2 i}(z) \varphi^{2 i}(3 z)$ at certain cusps

\begin{tabular}{c|cccccc} 
cusp & $\infty$ & 1 & $1 / 2$ & $1 / 3$ & $1 / 4$ & $1 / 6$ \\
\hline term & 1 & $(-z-1)^{2 k} \frac{(-1)^{k}}{2^{2 k} 3^{i}}$ & 0 & $(-3 z-1)^{2 k} \frac{(-1)^{i+k}}{2^{2 k}}$ & $(-4 z-1)^{2 k} \frac{(-1)^{i}}{3^{i}}$ & 0
\end{tabular}


By 4.1.4, we have

$$
\begin{aligned}
& v_{1 / 2}\left(\varphi^{4 k-2 i}(z) \varphi^{2 i}(3 z)\right)=3 k-i>0 \\
& v_{1 / 6}\left(\varphi^{4 k-2 i}(z) \varphi^{2 i}(3 z)\right)=k+i>0
\end{aligned}
$$

for all $1 \leq i \leq 2 k$, that is, the first terms of the Fourier series expansions of $\varphi^{4 k-2 i}(z) \varphi^{2 i}(3 z)$ at cusps $1 / 2$ and $1 / 6$ are 0 . This completes Table 6.1.1.

\subsection{Fourier Series Expansions of $E_{2 k}(t z)$ at Cer- tain Cusps}

We deduce the following theorem as an analogue of [40, Proposition 2.1] for the Eisenstein series $E_{2 k}(t z)$. Results of this section are new. For convenience we set $E_{(2 k, t)}(z)=E_{2 k}(t z)$ for $t \in \mathbb{N}$.

Theorem 6.2.1. Let $k \geq 2$ be an integer and $t \in \mathbb{N}$. The Fourier series expansion of $E_{(2 k, t)}(z)$ at the cusp $1 / c \in \mathbb{Q}$ is

$$
E_{(2 k, t)}\left(A_{c}^{-1} z\right)=\left(\frac{g}{t}\right)^{2 k}(-c z-1)^{2 k} E_{2 k}\left(\frac{g^{2}}{t} z+\frac{y g}{t}\right)
$$

where $g=\operatorname{gcd}(t, c), y$ is an integer and $A_{c}$ is given in 6.1.1).

Proof. The Fourier series expansion of $E_{(2 k, t)}(z)$ at the cusp $1 / c$ is given by 
the Fourier series expansion of $E_{(2 k, t)}\left(A_{c}^{-1} z\right)$ at the cusp $\infty$. We have

$$
E_{(2 k, t)}\left(A_{c}^{-1} z\right)=E_{(2 k, t)}\left(\frac{-z}{-c z-1}\right)=E_{2 k}\left(\frac{-t z}{-c z-1}\right)=E_{2 k}(\gamma z)
$$

where $\gamma=\left(\begin{array}{cc}-t & 0 \\ -c & -1\end{array}\right)$. As $\operatorname{gcd}(t / g, c / g)=1$, there exist $y, v \in \mathbb{Z}$ such that $\frac{t}{g}(-v)+\frac{c}{g} y=1$. Thus $L=\left(\begin{array}{cc}-t / g & y \\ -c / g & v\end{array}\right) \in S L_{2}(\mathbb{Z})\left(=\Gamma_{0}(1)\right)$. Then since $E_{2 k}(z) \in M_{2}\left(\Gamma_{0}(1)\right)$ for all $k \geq 2$, we have

$$
\begin{aligned}
E_{(2 k, t)}\left(A_{c}^{-1} z\right) & =E_{2 k}\left(L L^{-1} \gamma z\right) \\
& =\left(-c\left(\frac{(-v t+c y) z+y}{t}\right)+v\right)^{2 k} E_{2 k}\left(\frac{(-v t+c y) z+y}{t / g}\right) \\
& =(g / t)^{2 k}\left(c \frac{v t-c y}{g} z+\frac{v t-c y}{g}\right)^{2 k} E_{2 k}\left(\frac{g^{2} z+y g}{t}\right) \\
& =(g / t)^{2 k}(-c z-1)^{2 k} E_{2 k}\left(\frac{g^{2}}{t} z+\frac{y g}{t}\right)
\end{aligned}
$$

which completes the proof.

It follows from Theorem 6.2.1 and Definition 2.3.3 that the first term of the Fourier series expansion of $E_{2 k}(t z)$ at the cusp $1 / c$ is

$$
\left(\frac{g}{t}\right)^{2 k}(-c z-1)^{2 k}\left(\frac{-B_{2 k}}{4 k}\right) .
$$




\section{Chapter 7}

\section{On an Extension of the Ramanujan-Mordell Theorem}

Let $k, i$ and $n$ be non-negative integers. Ramanujan [62] has stated a general formula for $N\left(1^{2 k} ; n\right)$ for all $k \geq 1$ which was proven by Mordell [53. In 2010, Lemire gave formulas for $N\left(1^{r}, 2^{s}, 4^{t} ; n\right)$ for $r \in \mathbb{N}, s, t \in \mathbb{N}_{0}$, $r+s+t=4 k$, see [45]. Let $k>1,0 \leq i \leq 2 k, n \geq 0$. In this chapter we will give formulas for

$$
N\left(1^{4 k-2 i}, 3^{2 i} ; n\right) .
$$

Results of this chapter has been submitted for publication, see [6]. Methods used in this chapter are applicable to other problems and sets a computational example.

By Theorem 2.2 .3 we have

$$
R\left(\Gamma_{0}(12)\right)=\{1,1 / 2,1 / 3,1 / 4,1 / 6,1 / 12\} .
$$


For $M=\left(\begin{array}{cc}1 & 12 \\ 1 & 0\end{array}\right) \in \Gamma_{0}(12)$, we have $M(\infty)=1 / 12$. That is $1 / 12$ and $\infty$ are equivalent cusps in $\Gamma_{0}(12)$. In the remainder of this chapter we use $\infty$ and $1 / 12$ interchangeably.

\subsection{An Extension of Ramanujan-Mordell The-}

\section{orem}

Recall that in Chapter 5 we defined the eta quotients $C_{j, k}(z)$ for $j, k \in \mathbb{N}$ as

$$
\begin{aligned}
C_{j, k}(z) & =\left(\frac{\eta^{10}(2 z) \eta^{5}(3 z) \eta(4 z) \eta^{2}(6 z)}{\eta^{15}(z) \eta^{3}(12 z)}\right)\left(\frac{\eta^{2}(2 z) \eta(3 z) \eta^{3}(12 z)}{\eta^{3}(z) \eta(4 z) \eta^{2}(6 z)}\right)^{j}\left(\frac{\eta^{6}(z) \eta(6 z)}{\eta^{3}(2 z) \eta^{2}(3 z)}\right)^{k} \\
& =q^{j}+\sum_{n=j+1}^{\infty} c_{j, k}(n) q^{n} .
\end{aligned}
$$

In the following theorem we give a basis for $M_{2 k}\left(\Gamma_{0}(12)\right)$ when $k \geq 2$.

Theorem 7.1.1. Let $k \geq 2$ be an integer.

(a) The family $\left\{C_{j, 2 k}(z)\right\}_{1 \leq j \leq 4 k-5}$ constitute a basis for $S_{2 k}\left(\Gamma_{0}(12)\right)$.

(b) The set of Eisenstein series

$$
\left\{E_{2 k}(z), E_{2 k}(2 z), E_{2 k}(3 z), E_{2 k}(4 z), E_{2 k}(6 z), E_{2 k}(12 z)\right\}
$$

constitute a basis for $E_{2 k}\left(\Gamma_{0}(12)\right)$.

(c) The set

$$
\left\{E_{2 k}(\delta z) \mid \delta=1,2,3,4,6,12\right\} \cup\left\{C_{j, 2 k}(z) \mid 1 \leq j \leq 4 k-5\right\}
$$


constitute a basis for $M_{2 k}\left(\Gamma_{0}(12)\right)$.

Proof. The parts (a), (b), (c) follows from Theorem 5.1.3, Example 3.3.2 and (1.0.1), respectively.

Now we can state an extension of Ramanujan-Mordell Theorem using Theorem 7.1.1(c). For convenience we set

$$
\alpha_{k}=\frac{-4 k}{\left(2^{2 k}-1\right)\left(3^{2 k}-1\right) B_{2 k}}
$$

where $B_{2 k}$ are Bernoulli numbers given in Definition 3.1.1. Recall that we write $[j] f(z)=a_{j}$ for $f(z)=\sum_{n=0}^{\infty} a_{n} q^{n}$.

Theorem 7.1.2. Let $k, i, j \in \mathbb{N}$ such that $k>2,0 \leq i \leq 2 k$ and $1 \leq j \leq$ $4 k-5$. Let $\alpha_{k}$ be as in (7.1.2). Then

$$
\varphi^{4 k-2 i}(z) \varphi^{2 i}(3 z)=\sum_{r \mid 12} b_{(r, i, k)} E_{2 k}(r z)+\sum_{1 \leq j \leq 4 k-5} a_{(j, i, k)} C_{j, 2 k}(z)
$$

where

$$
\begin{aligned}
& b_{(1, i, k)}=(-1)^{k}\left(3^{2 k-i}+(-1)^{i+1}\right) \cdot \alpha_{k}, \\
& b_{(2, i, k)}=(-1)^{i+1}\left(1+(-1)^{i+k}\right)\left(3^{2 k-i}+(-1)^{i+1}\right) \cdot \alpha_{k}, \\
& b_{(3, i, k)}=(-1)^{i+k} 3^{2 k-i}\left(3^{i}+(-1)^{i+1}\right) \cdot \alpha_{k}, \\
& b_{(4, i, k)}=(-1)^{i} 2^{2 k}\left(3^{2 k-i}+(-1)^{i+1}\right) \cdot \alpha_{k}, \\
& b_{(6, i, k)}=-\left(1+(-1)^{i+k}\right) 3^{2 k-i}\left(3^{i}+(-1)^{i+1}\right) \cdot \alpha_{k},
\end{aligned}
$$




$$
b_{(12, i, k)}=2^{2 k} 3^{2 k-i}\left(3^{i}+(-1)^{i+1}\right) \cdot \alpha_{k},
$$

and

$$
a_{(j, i, k)}=N\left(1^{4 k-2 i}, 3^{2 i} ; j\right)-\sum_{r \mid 12} b_{(r, i, k)} \sigma_{2 k-1}(j / r)-\sum_{1 \leq l \leq j-1} a_{(l, i, k)} c_{l, 2 k}(j) .
$$

Proof. Let $k \geq 2$ be an integer and $i$ an integer with $0 \leq i \leq 2 k$. We write the following table of first terms of Fourier series expansions of $E_{2 k}(r z),(r \mid 12)$ and $\varphi^{4 k-2 i}(z) \varphi^{2 i}(3 z)$ from Table 6.1.1 and 6.2.1), respectively. Please note that we omit the $(-c z-1)^{2 k}$ part of first terms in the table below as they will not be necessary for calculations.

\begin{tabular}{|c|c|c|c|c|c|c|}
\hline cusp & $\infty$ & 1 & $1 / 2$ & $1 / 3$ & $1 / 4$ & $1 / 6$ \\
\hline$\varphi^{4 k-2 i}(z) \varphi^{2 i}(3 z)$ & 1 & $\frac{(-1)^{k}}{2^{2 k} 3^{i}}$ & 0 & $\frac{(-1)^{i+k}}{2^{2 k}}$ & $\frac{(-1)^{i}}{3^{i}}$ & 0 \\
\hline$E_{2 k}(z)$ & $-B_{2 k}$ & $-B_{2 k}$ & $-B_{2 k}$ & $-B_{2 k}$ & $-\stackrel{\breve{B}}{B}_{2 k}$ & $-B_{2 k}$ \\
\hline$E_{2 k}(2 z)$ & $\begin{array}{c}4 k \\
-B_{2 k} \\
4 k\end{array}$ & $\frac{-B_{2 k}}{4 k}\left(\frac{1}{2}\right)^{2 k}$ & $\begin{array}{c}4 k \\
-B_{2 k} \\
4 k\end{array}$ & $\frac{-B_{2 k}}{4 k}\left(\frac{1}{2}\right)^{2 k}$ & $\begin{array}{c}4 k \\
-B_{2 k} \\
4 k\end{array}$ & $\begin{array}{c}4 k \\
-B_{2 k} \\
4 k\end{array}$ \\
\hline$E_{2 k}(3 z)$ & $\frac{-B_{2 k}}{4 k}$ & $\frac{-B_{2 k}}{4 k}\left(\frac{1}{3}\right)^{2 k}$ & $\frac{-B_{2 k}}{4 k}\left(\frac{1}{3}\right)^{2 k}$ & $\frac{-B_{2 k}}{4 k}$ & $\frac{-B_{2 k}}{4 k}\left(\frac{1}{3}\right)^{2 k}$ & $\frac{-B_{2 k}}{4 k}$ \\
\hline$E_{2 k}(4 z)$ & $\frac{-B_{2 k}}{4 k}$ & $\frac{-B_{2 k}}{4 k}\left(\frac{1}{4}\right)^{2 k}$ & $\frac{-B_{2 k}}{4 k}\left(\frac{1}{2}\right)^{2 k}$ & $\frac{-B_{2 k}}{4 k}\left(\frac{1}{4}\right)^{2 k}$ & $\frac{-B_{2 k}}{4 k}$ & $\frac{-B_{2 k}}{4 k}\left(\frac{1}{2}\right)^{2 k}$ \\
\hline$E_{2 k}(6 z)$ & $\frac{-B_{2 k}}{4 k}$ & $\frac{-B_{2 k}}{4 k}\left(\frac{1}{6}\right)^{2 k}$ & $\frac{-B_{2 k}}{4 k}\left(\frac{1}{3}\right)^{2 k}$ & $\frac{-B_{2 k}}{4 k}\left(\frac{1}{2}\right)^{2 k}$ & $\frac{-B_{2 k}}{4 k}\left(\frac{1}{3}\right)^{2 k}$ & $\frac{-B_{2 k}}{4 k}$ \\
\hline$E_{2 k}(12 z)$ & $\frac{-B_{2 k}}{4 k}$ & $\frac{-B_{2 k}}{4 k}\left(\frac{1}{12}\right)^{2 k}$ & $\frac{-B_{2 k}}{4 k}\left(\frac{1}{6}\right)^{2 k}$ & $\frac{-B_{2 k}}{4 k}\left(\frac{1}{4}\right)^{2 k}$ & $\frac{-B_{2 k}}{4 k}\left(\frac{1}{3}\right)^{2 k}$ & $\frac{-B_{2 k}}{4 k}\left(\frac{1}{2}\right)^{2 k}$ \\
\hline
\end{tabular}

Table 7.1.1: First terms of $\varphi^{4 k-2 i}(z) \varphi^{2 i}(3 z)$ at cusps of $\Gamma_{0}(12)$ 
By 1.0.4) we have

$$
\varphi^{4 k-2 i}(z) \varphi^{2 i}(3 z)=\frac{\eta^{20 k-10 i}(2 z)}{\eta^{8 k-4 i}(z) \eta^{8 k-4 i}(4 z)} \cdot \frac{\eta^{10 i}(6 z)}{\eta^{4 i}(3 z) \eta^{4 i}(12 z)}
$$

By Theorem 4.2.6, we have $\varphi^{4 k-2 i}(z) \varphi^{2 i}(3 z) \in M_{2 k}\left(\Gamma_{0}(12)\right)$. By 1.0.1 and Theorem 7.1.1 we have

$$
\varphi^{4 k-2 i}(z) \varphi^{2 i}(3 z)=\sum_{r \mid 12} b_{(r, i, k)} E_{2 k}(r z)+\sum_{1 \leq j \leq 4 k-5} a_{(j, i, k)} C_{j, 2 k}(z)
$$

for some constants $b_{(1, i, k)}, b_{(2, i, k)}, b_{(3, i, k)}, b_{(4, i, k)}, b_{(6, i, k)}, b_{(12, i, k)}, a_{(1, i, k)}, \ldots, a_{(4 k-5, i, k)}$. Since $C_{1, k}(z), \ldots, C_{4 k-5, k}(z)$ are cusp forms, the first terms of their Fourier series expansions at all cusps are 0.

By appealing to Table 7.1.1 we equate the first terms of the Fourier series expansions of 7.1.10 in both sides at cusps $\infty, 1,1 / 2,1 / 3,1 / 4,1 / 6$ to obtain the system of linear equations

$$
\begin{aligned}
& b_{(1, i, k)}+b_{(2, i, k)}+b_{(3, i, k)}+b_{(4, i, k)}+b_{(6, i, k)}+b_{(12, i, k)}=\frac{-4 k}{B_{2 k}}, \\
& b_{(1, i, k)}+\frac{b_{(2, i, k)}}{2^{2 k}}+\frac{b_{(3, i, k)}}{3^{2 k}}+\frac{b_{(4, i, k)}}{4^{2 k}}+\frac{b_{(6, i, k)}}{6^{2 k}}+\frac{b_{(12, i, k)}}{12^{2 k}}=\frac{(-1)^{k}}{2^{2 k} 3^{i}} \cdot \frac{-4 k}{B_{2 k}}, \\
& b_{(1, i, k)}+b_{(2, i, k)}+\frac{b_{(3, i, k)}}{3^{2 k}}+\frac{b_{(4, i, k)}}{2^{2 k}}+\frac{b_{(6, i, k)}}{3^{2 k}}+\frac{b_{(12, i, k)}}{6^{2 k}}=0, \\
& b_{(1, i, k)}+\frac{b_{(2, i, k)}}{2^{2 k}}+b_{(3, i, k)}+\frac{b_{(4, i, k)}}{4^{2 k}}+\frac{b_{(6, i, k)}}{2^{2 k}}+\frac{b_{(12, i, k)}}{4^{2 k}}=\frac{(-1)^{i+k}}{2^{2 k}} \cdot \frac{-4 k}{B_{2 k}}, \\
& b_{(1, i, k)}+b_{(2, i, k)}+\frac{b_{(3, i, k)}}{3^{2 k}}+b_{(4, i, k)}+\frac{b_{(6, i, k)}}{3^{2 k}}+\frac{b_{(12, i, k)}}{3^{2 k}}=\frac{(-1)^{i}}{3^{i}} \cdot \frac{-4 k}{B_{2 k}}, \\
& b_{(1, i, k)}+b_{(2, i, k)}+b_{(3, i, k)}+\frac{b_{(4, i, k)}}{2^{2 k}}+b_{(6, i, k)}+\frac{b_{(12, i, k)}}{2^{2 k}}=0 .
\end{aligned}
$$


Solving the above system of linear equations, we obtain the asserted expressions for $b_{(r, i, k)}$ for $r=1,2,3,4,6,12$ in 7.1.3 (7.1.8). By Lemma 5.1.1, we have $[j] C_{j, k}(z)=1$ for each $j$ with $1 \leq j \leq 4 k-5$. Equating the coefficients of $q^{j}$ in both sides of (7.1.1) we obtain

$$
N\left(1^{4 k-2 i}, 3^{2 i} ; j\right)=\sum_{r \mid 12} b_{(r, i, k)} \sigma_{2 k-1}(j / r)+\sum_{1 \leq l \leq j-1} a_{(l, i, k)} c_{l, 2 k}(z)+a_{(j, i, k)} .
$$

We isolate $a_{(j, i, k)}$ to complete the proof of Theorem 7.1.2.

The following theorem follows immediately from Theorem 7.1.2.

Theorem 7.1.3. Let $k \geq 2$ be an integer and $i$ be an integer with $0 \leq i \leq 2 k$. Then

$$
N\left(1^{4 k-2 i}, 3^{2 i} ; n\right)=\sum_{r \mid 12} b_{(r, i, k)} \sigma_{2 k-1}(n / r)+\sum_{1 \leq j \leq 4 k-5} a_{(j, i, k)} c_{j, 2 k}(n),
$$

where $a_{(j, i, k)}, c_{j, 2 k}(n)$ and $b_{(r, i, k)}(r=1,2,3,4,6,12)$ are given in (7.1.9), 7.1.1), and (7.1.3)-7.1.8, respectively.

\subsection{Examples and Remarks}

We now illustrate Theorems 7.1 .2 and 7.1 .3 by some examples.

Example 7.2.1. We determine $N\left(1^{6}, 3^{2} ; n\right)$ for all $n \in \mathbb{N}$. We take $k=2$ 
and $i=1$ in Theorems 7.1.2 and 7.1.3. By $7.1 .3-7.1 .8$ we have

$$
\left\{\begin{array}{l}
b_{(1,1,2)}=28 / 5, b_{(2,1,2)}=0, b_{(3,1,2)}=-108 / 5 \\
b_{(4,1,2)}=-448 / 5, b_{(6,1,2)}=0, b_{(12,1,2)}=1728 / 5
\end{array}\right.
$$

We first compute $N\left(1^{6}, 3^{2} ; n\right)$ for $n=1,2,3$ as $4 k-5=3$, and obtain

$$
N\left(1^{6}, 3^{2} ; 1\right)=12, N\left(1^{6}, 3^{2} ; 2\right)=60, N\left(1^{6}, 3^{2} ; 3\right)=164
$$

By (7.1.9), (7.2.1) and (7.2.2), we obtain

$$
a_{(1,1,2)}=32 / 5, a_{(2,1,2)}=48, a_{(3,1,2)}=576 / 5 \text {. }
$$

Then, by Theorem 7.1.3, for all $n \in \mathbb{N}$, we have

$$
\begin{aligned}
N\left(1^{6}, 3^{2} ; n\right)= & \frac{28}{5} \sigma_{3}(n)-\frac{108}{5} \sigma_{3}(n / 3)-\frac{448}{5} \sigma_{3}(n / 4)+\frac{1728}{5} \sigma_{3}(n / 12) \\
& +\frac{32}{5} c_{1,4}(n)+48 c_{2,4}(n)+\frac{576}{5} c_{3,4}(n),
\end{aligned}
$$

which agrees with the known results, see for example [14]. We note that the last two coefficients in the above expression are different from the ones in [14] since we used a different basis for the space of cusp forms.

Example 7.2.2. We determine $N\left(1^{4}, 3^{8} ; n\right)$ for all $n \in \mathbb{N}$. We take $k=3$ 
and $i=4$ in Theorems 7.1.2 and 7.1.3. By 7.1.3 7.1 .8 we have

$$
\left\{\begin{array}{l}
b_{(1,4,3)}=8 / 91, b_{(2,4,3)}=0, b_{(3,4,3)}=720 / 91 \\
b_{(4,4,3)}=-512 / 91, b_{(6,4,3)}=0, b_{(12,4,3)}=-46080 / 91
\end{array}\right.
$$

We first compute $N\left(1^{4}, 3^{8} ; n\right)$ for $n=1,2,3,4,5,6,7$ as $4 k-5=7$, and obtain

$$
\left\{\begin{array}{l}
N\left(1^{4}, 3^{8} ; 1\right)=8, N\left(1^{4}, 3^{8} ; 2\right)=24, N\left(1^{4}, 3^{8} ; 3\right)=48 \\
N\left(1^{4}, 3^{8} ; 4\right)=152, N\left(1^{4}, 3^{8} ; 5\right)=432 \\
N\left(1^{4}, 3^{8} ; 6\right)=720, N\left(1^{4}, 3^{8} ; 7\right)=1344
\end{array}\right.
$$

By (7.1.9), (7.2.3) and (7.2.4), we obtain

$$
\begin{aligned}
& a_{(1,4,3)}=720 / 91, a_{(2,4,3)}=14880 / 91, a_{(3,4,3)}=123376 / 91 a_{(4,4,3)}=40640 / 7, \\
& a_{(5,4,3)}=1248448 / 91, a_{(6,4,3)}=1551360 / 91, a_{(7,4,3)}=792576 / 91 .
\end{aligned}
$$

Then, by Theorem 7.1.3, for all $n \in \mathbb{N}$, we have

$$
\begin{aligned}
N\left(1^{4}, 3^{8} ; n\right)= & \frac{8}{91} \sigma_{5}(n)+\frac{720}{91} \sigma_{5}(n / 3)-\frac{512}{91} \sigma_{5}(n / 4)-\frac{46080}{91} \sigma_{5}(n / 12) \\
& +\frac{720}{91} c_{1,6}(n)+\frac{14880}{91} c_{2,6}(n)+\frac{123376}{91} c_{3,6}(n)+\frac{40640}{7} c_{4,6}(n) \\
& +\frac{1248448}{91} c_{5,6}(n)+\frac{1551360}{91} c_{6,6}(n)+\frac{792576}{91} c_{7,6}(n),
\end{aligned}
$$

which agrees with the known results, see for example [3]. 
Example 7.2.3. Ramanujan [62] stated a formula for $N\left(1^{2 k}, 3^{0} ; n\right)$, which was proved by Mordell in [53], see also [20, 12]. By taking $i=0$ in Theorems 7.1 .2 and 7.1 .3 , we obtain

$$
\begin{array}{r}
N\left(1^{4 k}, 3^{0} ; n\right)=\frac{4 k}{\left(2^{2 k}-1\right) B_{2 k}}\left((-1)^{k+1} \sigma_{2 k-1}(n)+\left(1+(-1)^{k}\right) \sigma_{2 k-1}(n / 2)\right. \\
\left.-2^{2 k} \sigma_{2 k-1}(n / 4)\right)+\sum_{1 \leq j \leq 4 k-5} a_{(j, 0, k)} c_{j, 2 k}(n),
\end{array}
$$

where $a_{(j, 0, k)}$ and $c_{j, 2 k}(n)$ are given by 7.1 .9 and $(7.1 .1$, respectively. The coefficients of $\sigma_{2 k-1}(n / r)(r=1,2,4)$ in the above formula agree with those in [20, Theorem 1.1] and [12, Theorem 4.1]. Different coefficients in the cusp part are due to the choice of our basis for the space $S_{2 k}\left(\Gamma_{0}(12)\right)$ of cusp forms. 


\section{Chapter 8}

\section{Generating Functions for Number of Algebraic Points on an Elliptic Curve on Prime Characteristic Fields}

Recall that if $f(z) \in S_{k}\left(\Gamma_{0}(N), \chi\right)$, then we have $f(t \cdot z) \in S_{k}\left(\Gamma_{0}(t \cdot N), \chi\right)$. The subspace of cusp forms arising from this property is called old subspace of cusp forms and denoted by $S_{k}^{\text {old }}\left(\Gamma_{0}(N), \chi\right)$, see [70, Definition 8.27]. And the space of newforms $S_{k}^{n e w}\left(\Gamma_{0}(N), \chi\right)$ is the subspace that satisfies the decomposition, see [70, p. 162]

$$
S_{k}\left(\Gamma_{0}(N), \chi\right)=S_{k}^{\text {old }}\left(\Gamma_{0}(N), \chi\right) \oplus S_{k}^{\text {new }}\left(\Gamma_{0}(N), \chi\right)
$$

Martin and Ono [49] listed all the weight 2 newforms that are eta quotients, and gave their corresponding strong Weil curves. There are such eta quotients only for $M_{2}\left(\Gamma_{0}(N)\right)$ with $N=11,14,15,20,24,27,32,36,48,64,80,144$. 
Recently in [59], newforms in $M_{2}\left(\Gamma_{0}(N)\right)$ with $N=33,40,42,70$ are given as linear combinations of eta quotients. In [2], congruences between Fourier coefficients of some newforms is given.

In this chapter we express the newforms in $M_{2}\left(\Gamma_{0}(N)\right)$ with $N=11,14$, 15, 20, 24, 27, 30, 32, 33, 34, 40, 42, 44, 45, 56, 64 as linear combinations of Eisenstein series and eta quotients, and give their corresponding strong Weil curves. Let $p$ denote a prime and $E\left(\mathbb{Z}_{p}\right)$ denote the group of algebraic points of an elliptic curve $E$ over $\mathbb{Z}_{p}$. We give a generating function for the order of $E\left(\mathbb{Z}_{p}\right)$ for certain strong Weil curves in terms of Eisenstein series and eta quotients. We then use our generating functions to deduce congruence relations for the order of $E\left(\mathbb{Z}_{p}\right)$ for those strong Weil curves.

\subsection{Newforms in Terms of Eisenstein Series and Eta Quotients}

Theorem 8.1.1. Let $N \in\{11,14,15,20,24,27,30,32,33,34,40,42,44,45,56,64\}$.

In Table 8.1.1 below we express the newforms $F_{N}(z)$ in $M_{2}\left(\Gamma_{0}(N)\right)$ as linear combinations of Eisenstein series and eta quotients. 
Table 8.1.1: Newforms in $M_{2}\left(\Gamma_{0}(N)\right)$

\begin{tabular}{|c|c|c|}
\hline Level & & Eisenstein series and eta quotients \\
\hline \multirow[t]{2}{*}{11} & $F_{11}(z)=$ & $-5 \eta_{22}[-2,4,-2,4](z)-4 \eta_{22}[0,2,0,2](z)$ \\
\hline & & $+\frac{1}{2} L_{2}(z)+L_{11}(z)-\frac{1}{2} L_{22}(z)$ \\
\hline 14 & $F_{14}(z)=$ & $-3 \eta_{14}[-2,4,-2,4](z)+\frac{1}{2} L_{2}(z)+L_{7}(z)-\frac{1}{2} L_{14}(z)$ \\
\hline 15 & $F_{15}(z)=$ & $-4 \eta_{15}[-1,3,-1,3](z)+\frac{1}{3} L_{3}(z)+L_{5}(z)-\frac{1}{3} L_{15}(z)$ \\
\hline \multirow[t]{2}{*}{20} & $F_{20}(z)=$ & $-6 \eta_{20}[0,-2,4,0,-2,4](z)$ \\
\hline & & $+\frac{3}{2} L_{2}(z)-\frac{1}{2} L_{4}(z)-L_{5}(z)+\frac{3}{2} L_{10}(z)-\frac{1}{2} L_{20}(z)$ \\
\hline \multirow[t]{2}{*}{24} & $F_{24}(z)=$ & $-2 \eta_{24}[4,-2,0,0,0,0,-2,4](z)+\frac{3}{2} L_{2}(z)+L_{3}(z)$ \\
\hline & & $+\frac{3}{2} L_{4}(z)-\frac{3}{2} L_{6}(z)-L_{8}(z)-\frac{3}{2} L_{12}(z)+L_{24}(z)$ \\
\hline \multirow[t]{2}{*}{27} & $F_{27}(z)=$ & $-9 \eta_{27}[0,-1,2,3](z)+\frac{2}{3} L_{3}(z)-\frac{1}{6} L_{9}(z)$ \\
\hline & & $+\frac{1}{2} E_{2, \chi_{-3}, \chi_{-3}}(z)$ \\
\hline \multirow[t]{2}{*}{30} & $F_{30}(z)=$ & $6 \eta_{30}[1,-1,1,-1,-1,3,-1,3](z)+2 L_{2}(z)+L_{3}(z)$ \\
\hline & & $+\frac{1}{5} L_{5}(z)-2 L_{6}(z)-\frac{2}{5} L_{10}(z)-\frac{1}{5} L_{15}(z)+\frac{2}{5} L_{30}(z)$ \\
\hline 32 & $F_{32}(z)=$ & $-8 \eta_{32}[0,0,-2,4,-2,4](z)+\frac{3}{4} L_{2}(z)-\frac{1}{4} L_{4}(z)$ \\
\hline
\end{tabular}




\begin{tabular}{|c|c|c|}
\hline \multicolumn{2}{|l|}{ Level } & \multirow[t]{2}{*}{ Eisenstein series and eta quotients } \\
\hline & & \\
\hline \multirow[t]{2}{*}{33} & $F_{33}(z)=$ & $-10 \eta_{33}[-1,3,-1,3](z)-6 \eta_{33}[0,2,0,2](z)$ \\
\hline & & $-2 \eta_{33}[1,1,1,1](z)+\frac{1}{3} L_{3}(z)+L_{11}(z)-\frac{1}{3} L_{33}(z)$ \\
\hline \multirow[t]{3}{*}{34} & $F_{34}(z)=$ & $6 \eta_{68}[0,-2,4,0,-2,4](z)$ \\
\hline & & $+\frac{3}{8} \eta_{68}[-4,10,-4,-4,10,-4](z)$ \\
\hline & & $-\frac{1}{2} L_{2}(z)+\frac{1}{2} L_{17}(z)-\frac{1}{2} L_{34}(z)$ \\
\hline \multirow[t]{2}{*}{40} & $F_{40}(z)=$ & $-4 \eta_{40}[2,-1,-1,2,2,-1,-1,2](z)+\frac{3}{2} L_{2}(z)+\frac{3}{2} L_{4}(z)$ \\
\hline & & $+L_{5}(z)-L_{8}(z)-\frac{3}{2} L_{10}(z)-\frac{3}{2} L_{20}(z)+L_{40}(z)$ \\
\hline \multirow[t]{4}{*}{42} & $F_{42}(z)=$ & $-8 \eta_{42}[-1,1,1,-1,2,0,0,2](z)$ \\
\hline & & $-8 \eta_{42}[1,-1,-1,1,0,2,2,0](z)$ \\
\hline & & $+L_{2}(z)-L_{3}(z)+L_{6}(z)+\frac{1}{7} L_{7}(z)$ \\
\hline & & $-\frac{1}{7} L_{14}(z)+\frac{1}{7} L_{21}(z)-\frac{1}{7} L_{42}(z)$ \\
\hline \multirow[t]{2}{*}{44} & $F_{44}(z)=$ & $3 \eta_{44}[-1,0,3,3,0,-1](z)-3 \eta_{44}[3,0,-1,-1,0,3](z)$ \\
\hline & & $-2 L_{4}(z)+2 L_{11}(z)-2 L_{44}(z)$ \\
\hline 45 & $F_{45}(z)=$ & $2 \eta_{45}[-1,1,2,2,1,-1](z)-2 \eta_{45}[2,1,-1,-1,1,2](z)$ \\
\hline
\end{tabular}




\begin{tabular}{|c|c|c|}
\hline \multicolumn{2}{|l|}{ Level } & \multirow{2}{*}{$\begin{array}{l}\text { Eisenstein series and eta quotients } \\
+L_{5}(z)-\frac{2}{3} L_{9}(z)-\frac{2}{5} L_{15}(z)-\frac{14}{15} L_{45}(z)\end{array}$} \\
\hline & & \\
\hline \multirow[t]{3}{*}{$56 A$} & $F_{56 A}(z)=$ & $-4 \eta_{56}[0,-1,1,0,0,3,1,0](z)$ \\
\hline & & $-2 \eta_{56}[0,-4,10,0,-4,0,-2,4](z)$ \\
\hline & & $+\frac{3}{2} L_{2}(z)-\frac{1}{2} L_{4}(z)+\frac{3}{7} L_{7}(z)-\frac{9}{14} L_{14}(z)+\frac{3}{14} L_{28}(z)$ \\
\hline \multirow[t]{3}{*}{$56 B$} & $F_{56 B}(z)=$ & $-4 \eta_{56}[0,-2,3,0,1,2,-1,1](z)$ \\
\hline & & $+2 \eta_{56}[0,1,2,0,-1,-3,6,-1](z)$ \\
\hline & & $+\frac{3}{2} L_{2}(z)-\frac{1}{2} L_{4}(z)-L_{7}(z)+\frac{3}{2} L_{14}(z)-\frac{1}{2} L_{28}(z)$ \\
\hline \multirow[t]{2}{*}{64} & $F_{64}(z)=$ & $-4 \eta_{64}[0,0,-4,9,-2,-1,2](z)+\frac{3}{4} L_{2}(z)-\frac{1}{4} L_{4}(z)$ \\
\hline & & $+\frac{1}{2} E_{2, \chi_{-4}, \chi_{-4}}(z)$ \\
\hline
\end{tabular}

Proof. We give the proofs only for levels 30 and 34 as the rest can be proven similarly. Appealing to Theorem 4.2.6 one can show that each $\eta_{N}[*](z)$ given in Table 8.1.1 are in $M_{2}\left(\Gamma_{0}(N)\right)$.

Recall that we use the Sturm bound $S(2 k, N)$ defined by 2.4.1) to show the equality of two modular forms in the same modular space.

By 2.4.1) we obtain

$$
S(2,30)=12, S(2,34)=9 \text {. }
$$


In [27, Table 3] all newforms in $M_{2}\left(\Gamma_{0}(N)\right)$ with $N$ less than 1000 have been given by listing their Fourier coefficients for primes up to 100. Using the results from [27, p. 25] together with [27, Table 3] we determine the first $S(2, N)+1$ terms of the Fourier series expansions of all the newforms in $M_{2}\left(\Gamma_{0}(N)\right)$ with $N=30,34$. We give them in Table 8.1 .2 below.

Table 8.1.2: First $S(2, N)+1$ terms of the Fourier series expansions of the newforms in $M_{2}\left(\Gamma_{0}(N)\right)$

\begin{tabular}{ll}
\hline \hline$N$ & First $S(2, N)+1$ terms of the newforms in level $N$ \\
\hline \hline 30 & $q-q^{2}+q^{3}+q^{4}-q^{5}-q^{6}-4 q^{7}-q^{8}+q^{9}+q^{10}+q^{12}+O\left(q^{13}\right)$, \\
34 & $q+q^{2}-2 q^{3}+q^{4}-2 q^{6}-4 q^{7}+q^{8}+O\left(q^{9}\right)$ \\
\hline
\end{tabular}

Let us consider the function $F_{30}(z)$ from Table 8.1.1. By Theorem 3.2.1. 4.2.6 and Lemma 2.4.2, we have $F_{30}(z) \in M_{2}\left(\Gamma_{0}(30)\right)$. Using MAPLE we calculate the first $S(2,30)+1=13$ terms of the Fourier series expansion of $F_{30}(z)$ as

$$
F_{30}(z)=q-q^{2}+q^{3}+q^{4}-q^{5}-q^{6}-4 q^{7}-q^{8}+q^{9}+q^{10}+q^{12}+O\left(q^{13}\right) .
$$

Since the first 13 terms of the newform of $M_{2}\left(\Gamma_{0}(30)\right)$ in Table 8.1 .2 are the same as the first 13 terms of $F_{30}(z)$, it follows from Theorem 2.4.4 that $F_{30}(z)$ is equal to the newform of weight 2 and level 30 .

There are no eta quotients in $M_{2}\left(\Gamma_{0}(34)\right)$. The newform of $M_{2}\left(\Gamma_{0}(34)\right)$ 
is also in $M_{2}\left(\Gamma_{0}(68)\right)$, since $M_{2}\left(\Gamma_{0}(34)\right) \subseteq M_{2}\left(\Gamma_{0}(68)\right)$. See [35] for a similar idea.

By Theorem 3.2.1, 4.2.6) and Lemma 2.4.2, we have $F_{34}(z) \in M_{2}\left(\Gamma_{0}(68)\right)$. Using MAPLE we calculate the first $S(2,34)+1=10$ terms of the Fourier series expansion of $F_{34}(z)$ as

$$
F_{34}(z)=q+q^{2}-2 q^{3}+q^{4}-2 q^{6}-4 q^{7}+q^{8}+q^{9}+O\left(q^{10}\right)
$$

which matches with the first 10 terms of Fourier series expansion of the newform in $M_{2}\left(\Gamma_{0}(34)\right)$ given in Table 8.1.2, Then it follows from Theorem 2.4 .4 that $F_{34}(z)$ is equal to the newform in $M_{2}\left(\Gamma_{0}(34)\right)$. The remaining cases can be proven similarly. Note that there are two different newforms in $M_{2}\left(\Gamma_{0}(56)\right)$, and following the notation from [27, Table 3] we label them as $F_{56 A}(z)$ and $F_{56 B}(z)$ in Table 8.1.1.

Note that in Table 8.1.1 we gave alternative representations for weight 2 newforms in levels 11, 14, 15, 20, 24, 27, 32, 64 in terms of Eisenstein series and eta quotients. These were previously given as single eta quotients in [49]. Additionally we have obtained newforms in $M_{2}\left(\Gamma_{0}(N)\right)$ for various $N<100$. These results have been presented in Appendix B. 


\subsection{Some Arithmetic Properties of $\left|E\left(\mathbb{Z}_{p}\right)\right|$ for Certain Elliptic Curves}

We recall that $p$ denotes a prime. We first state the $a_{p}$ version of the modularity theorem, see [28, Theorem 8.8.1], which gives a relation between $\left|E\left(\mathbb{Z}_{p}\right)\right|$ and the Fourier coefficients of the corresponding newform.

Theorem 8.2.1. (Modularity Theorem, Version $a_{p}$ ) Let $E$ be an elliptic curve over $\mathbb{Q}$ with conductor $N$ given by

$$
y^{2}+a_{1} x y+a_{3} y=x^{3}+a_{2} x^{2}+a_{4} x+a_{6},
$$

where $a_{1}, a_{2}, a_{3}, a_{4}, a_{6} \in \mathbb{Z}$. Let

$E\left(\mathbb{Z}_{p}\right)=\{\infty\} \cup\left\{(x, y) \in \mathbb{Z}_{p} \times \mathbb{Z}_{p} \mid y^{2}+a_{1} x y+a_{3} y=x^{3}+a_{2} x^{2}+a_{4} x+a_{6}\right\}$

Then for some newform $f_{E} \in S_{2}\left(\Gamma_{0}(N)\right)$ we have

$$
\left|E\left(\mathbb{Z}_{p}\right)\right|=p+1-[p] f_{E}(z) \text { for } p \nmid N
$$

We deduce the following theorem from [27, Table 1].

Theorem 8.2.2. Table 8.2.1 below is a list of elliptic curves, more specifically strong Weil curves, corresponding to the newforms given in Table 8.1.1. 
Table 8.2.1. $y^{2}+a_{1} x y+a_{3} y=x^{3}+a_{2} x^{2}+a_{4} x+a_{6}$

\begin{tabular}{|c|c|c|c|c|c|c|}
\hline Newform & Strong Weil curve & $a_{1}$ & $a_{2}$ & $a_{3}$ & $a_{4}$ & $a_{6}$ \\
\hline$F_{11}(z)$ & $E_{11 A}$ & 0 & -1 & 1 & -10 & -20 \\
\hline$F_{14}(z)$ & $E_{14 A}$ & 1 & 0 & 1 & 4 & -6 \\
\hline$F_{15}(z)$ & $E_{15 A}$ & 1 & 1 & 1 & -10 & -10 \\
\hline$F_{20}(z)$ & $E_{20 A}$ & 0 & 1 & 0 & 4 & 4 \\
\hline$F_{24}(z)$ & $E_{24 A}$ & 0 & -1 & 0 & -4 & 4 \\
\hline$F_{27}(z)$ & $E_{27 A}$ & 0 & 0 & 1 & 0 & -7 \\
\hline$F_{30}(z)$ & $E_{30 A}$ & 1 & 0 & 1 & 1 & 2 \\
\hline$F_{32}(z)$ & $E_{32 A}$ & 0 & 0 & 0 & 4 & 0 \\
\hline$F_{33}(z)$ & $E_{33 A}$ & 1 & 1 & 0 & -11 & 0 \\
\hline$F_{34}(z)$ & $E_{34 A}$ & 1 & 0 & 0 & -3 & 1 \\
\hline$F_{40}(z)$ & $E_{40 A}$ & 0 & 0 & 0 & -7 & -6 \\
\hline$F_{42}(z)$ & $E_{42 A}$ & 1 & 1 & 1 & -4 & 5 \\
\hline$F_{44}(z)$ & $E_{44 A}$ & 0 & 1 & 0 & 3 & -1 \\
\hline$F_{45}(z)$ & $E_{45 A}$ & 1 & -1 & 0 & 0 & -5 \\
\hline$F_{56 A}(z)$ & $E_{56 A}$ & 0 & 0 & 0 & 1 & 2 \\
\hline$F_{56 B}(z)$ & $E_{56 B}$ & 0 & -1 & 0 & 0 & -4 \\
\hline$F_{64}(z)$ & $E_{64 A}$ & 0 & 0 & 0 & -4 & 0 \\
\hline
\end{tabular}

We use Theorems 8.1.1, 8.2.1 and 8.2.2 to give generating functions for the group order $\left|E_{N}\left(\mathbb{Z}_{p}\right)\right|$ of the elliptic curves in Table 8.2.1. 
Theorem 8.2.3. Consider the elliptic curves listed in Table 8.2.1. We have

$$
\begin{aligned}
&\left|E_{11 A}\left(\mathbb{Z}_{p}\right)\right|= 5[p]\left(\eta_{22}[-2,4,-2,4](z)\right) \text { for all } p \nmid 22, \\
&\left|E_{14 A}\left(\mathbb{Z}_{p}\right)\right|=3[p]\left(\eta_{14}[-2,4,-2,4](z)\right) \text { for all } p \nmid 14, \\
&\left|E_{15 A}\left(\mathbb{Z}_{p}\right)\right|=4[p]\left(\eta_{15}[-1,3,-1,3](z)\right) \text { for all } p \nmid 15, \\
&\left|E_{20 A}\left(\mathbb{Z}_{p}\right)\right|=6[p]\left(\eta_{20}[0,-2,4,0,-2,4](z)\right) \text { for all } p \nmid 20, \\
&\left|E_{24 A}\left(\mathbb{Z}_{p}\right)\right|=2[p]\left(\eta_{24}[4,-2,0,0,0,0,-2,4](z)\right) \text { for all } p \nmid 24, \\
&\left|E_{27 A}\left(\mathbb{Z}_{p}\right)\right|=\left(\frac{1-\chi-3(p)}{2}\right)(p+1) \\
&+9[p]\left(\eta_{27}[0,-1,2,3](z)\right) \text { for all } p \neq 3, \\
&\left|E_{30 A}\left(\mathbb{Z}_{p}\right)\right|=-6[p]\left(\eta_{30}[1,-1,1,-1,-1,3,-1,3](z)\right) \text { for all } p \nmid 30, \\
&\left|E_{32 A}\left(\mathbb{Z}_{p}\right)\right|=\left(\frac{1-\chi-4}{2}\right)(p) \\
&-8[p]\left(\eta_{32}[0,0,-2,4,-2,4](z)\right) \text { for all } p \neq 2, \\
&\left.-\eta_{44}[3,0,-1,-1,0,3](z)\right) \text { for all } p \nmid 44, \\
&\left|E_{33 A}\left(\mathbb{Z}_{p}\right)\right|=2[p]\left(5 \eta_{33}[-1,3,-1,3](z)+\eta_{33}[1,1,1,1](z)\right) \text { for all } p \nmid 33, \\
&\left|E_{34 A}\left(\mathbb{Z}_{p}\right)\right|= \frac{3(p+1)}{2}-3[p]\left(2 \eta_{68}[0,-2,4,0,-2,4](z)\right. \\
&\left.+\frac{1}{8} \eta_{68}[-4,10,-4,-4,10,-4](z)\right) \text { for all } p \nmid 34, \\
&\left.+\eta_{42}[1,-1,-1,1,0,2,2,0](z)\right) \text { for all } p \nmid 42, \\
&\left|E_{40 A}\left(\mathbb{Z}_{p}\right)\right|=4[p]\left(\eta_{40}[2,-1,-1,2,2,-1,-1,2](z)\right) \text { for all } p \nmid 40, \\
&\left|E_{42 A}\left(\mathbb{Z}_{p}\right)\right|=8[p]\left(\eta_{42}[-1,1,1,-1,2,0,0,2](z)\right. \\
&\left|E_{44 A}\left(\mathbb{Z}_{p}\right)\right|=3(p+1)-3[p]\left(\eta_{44}[-1,0,3,3,0,-1](z)\right. \\
&=
\end{aligned}
$$




$$
\begin{aligned}
\left|E_{45 A}\left(\mathbb{Z}_{p}\right)\right|=2 & (p+1)-2[p]\left(\eta_{45}[-1,1,2,2,1,-1](z)\right. \\
& \left.-\eta_{45}[2,1,-1,-1,1,2](z)\right) \text { for all } p \nmid 45, \\
\left|E_{56 A}\left(\mathbb{Z}_{p}\right)\right|=2[p]\left(2 \eta_{56}[0,-1,1,0,0,3,1,0](z)\right. & \left.+\eta_{56}[0,-4,10,0,-4,0,-2,4](z)\right) \text { for all } p \nmid 56, \\
\left|E_{56 B}\left(\mathbb{Z}_{p}\right)\right|=2[p]\left(2 \eta_{56}[0,-2,3,0,1,2,-1,1](z)\right. & \left.-\eta_{56}[0,1,2,0,-1,-3,6,-1](z)\right) \text { for all } p \nmid 56, \\
& \left(\frac{1-\chi_{-4}(p)}{2}\right)(p+1) \\
\left|E_{64 A}\left(\mathbb{Z}_{p}\right)\right|= & +4[p]\left(\eta_{64}[0,0,-4,9,-2,-1,2](z)\right) \text { for all } p \neq 2 .
\end{aligned}
$$

Proof. We just prove the equalities for $\left|E_{30 A}\left(\mathbb{Z}_{p}\right)\right|$ and $\left|E_{45 A}\left(\mathbb{Z}_{p}\right)\right|$ as the remaining ones can be proven similarly.

By Theorems 8.1.1, 8.2.1 and 8.2.2, for all $p \nmid 30$, we have,

$$
\begin{aligned}
\left|E_{30 A}\left(\mathbb{Z}_{p}\right)\right|= & +1-[p] F_{30 A}(z) \\
= & p+1-[p]\left(6 \frac{\eta(z) \eta(3 z) \eta^{3}(10 z) \eta^{3}(30 z)}{\eta(2 z) \eta(5 z) \eta(6 z) \eta(15 z)}+2 L_{2}(z)+L_{3}(z)\right. \\
& \left.\quad+\frac{1}{5} L_{5}(z)-2 L_{6}(z)-\frac{2}{5} L_{10}(z)-\frac{1}{5} L_{15}(z)+\frac{2}{5} L_{30}(z)\right) \\
= & p+1-6[p]\left(\frac{\eta(z) \eta(3 z) \eta^{3}(10 z) \eta^{3}(30 z)}{\eta(2 z) \eta(5 z) \eta(6 z) \eta(15 z)}\right)-\sigma(p) \\
= & -6[p]\left(\frac{\eta(z) \eta(3 z) \eta^{3}(10 z) \eta^{3}(30 z)}{\eta(2 z) \eta(5 z) \eta(6 z) \eta(15 z)}\right)
\end{aligned}
$$

which completes the proof of the equality for $\left|E_{30 A}\left(\mathbb{Z}_{p}\right)\right|$. 
By Theorems 8.1.1, 8.2.1 and 8.2.2, for all $p \nmid 45$, we have

$$
\begin{aligned}
&\left|E_{45 A}\left(\mathbb{Z}_{p}\right)\right|=p+1-[p] F_{45}(z) \\
&=p+1-[p]\left(2 \frac{\eta(3 z) \eta^{2}(5 z) \eta^{2}(9 z) \eta(15 z)}{\eta(z) \eta(45 z)}-2 \frac{\eta^{2}(z) \eta(3 z) \eta(15 z) \eta^{2}(45 z)}{\eta(5 z) \eta(9 z)}\right. \\
&\left.+L_{5}(z)-\frac{2}{3} L_{9}(z)-\frac{2}{5} L_{15}(z)-\frac{14}{15} L_{45}(z)\right) \\
&=p+1-2[p]\left(\frac{\eta(3 z) \eta^{2}(5 z) \eta^{2}(9 z) \eta(15 z)}{\eta(z) \eta(45 z)}-\frac{\eta^{2}(z) \eta(3 z) \eta(15 z) \eta^{2}(45 z)}{\eta(5 z) \eta(9 z)}\right)+\sigma(p) \\
&=2(p+1)-2[p]\left(\frac{\eta(3 z) \eta^{2}(5 z) \eta^{2}(9 z) \eta(15 z)}{\eta(z) \eta(45 z)}-\frac{\eta^{2}(z) \eta(3 z) \eta(15 z) \eta^{2}(45 z)}{\eta(5 z) \eta(9 z)}\right),
\end{aligned}
$$

which completes the proof of the equality for $\left|E_{45 A}\left(\mathbb{Z}_{p}\right)\right|$.

Let $E: y^{2}=x^{3}+x$. It is known that $\left|E\left(\mathbb{Z}_{p}\right)\right| \equiv 0(\bmod 4)$ for all $p \geq 3$, see [68, Exercise 5.12]. Below we give similar congruence relations for the number of algebraic points on various elliptic curves over $\mathbb{Z}_{p}$. These results are new and follow from Theorem 8.2.3.

Corollary 8.2.4. We have

$$
\begin{aligned}
& \left|E_{11 A}\left(\mathbb{Z}_{p}\right)\right| \equiv 0(\bmod 5) \text { for all } p \nmid 22, \\
& \left|E_{14 A}\left(\mathbb{Z}_{p}\right)\right| \equiv 0(\bmod 3) \text { for all } p \nmid 14, \\
& \left|E_{15 A}\left(\mathbb{Z}_{p}\right)\right| \equiv 0(\bmod 4) \text { for all } p \nmid 15, \\
& \left|E_{20 A}\left(\mathbb{Z}_{p}\right)\right| \equiv 0(\bmod 6) \text { for all } p \nmid 20, \\
& \left|E_{24 A}\left(\mathbb{Z}_{p}\right)\right| \equiv 0(\bmod 2) \text { for all } p \nmid 24, \\
& \left|E_{30 A}\left(\mathbb{Z}_{p}\right)\right| \equiv 0(\bmod 6) \text { for all } p \nmid 30,
\end{aligned}
$$




$$
\begin{aligned}
& \left|E_{33 A}\left(\mathbb{Z}_{p}\right)\right| \equiv 0(\bmod 2) \text { for all } p \nmid 33, \\
& \left|E_{34 A}\left(\mathbb{Z}_{p}\right)\right| \equiv 0(\bmod 3) \text { for all } p \nmid 34, \\
& \left|E_{40 A}\left(\mathbb{Z}_{p}\right)\right| \equiv 0(\bmod 4) \text { for all } p \nmid 40, \\
& \left|E_{42 A}\left(\mathbb{Z}_{p}\right)\right| \equiv 0(\bmod 8) \text { for all } p \nmid 42, \\
& \left|E_{44 A}\left(\mathbb{Z}_{p}\right)\right| \equiv 0(\bmod 3) \text { for all } p \nmid 44, \\
& \left|E_{45 A}\left(\mathbb{Z}_{p}\right)\right| \equiv 0(\bmod 2) \text { for all } p \nmid 45, \\
& \left|E_{56 A}\left(\mathbb{Z}_{p}\right)\right| \equiv 0(\bmod 2) \text { for all } p \nmid 56, \\
& \left|E_{56 B}\left(\mathbb{Z}_{p}\right)\right| \equiv 0(\bmod 2) \text { for all } p \nmid 56 .
\end{aligned}
$$




\section{Part III}

\section{Further Results and Future Research}




\section{Chapter 9}

\section{Bases for $M_{3}\left(\Gamma_{0}(24), \chi\right)$ and $M_{2}\left(\Gamma_{0}(108), \chi\right)$}

In this chapter we give bases for $M_{3}\left(\Gamma_{0}(24), \chi\right)$ and $M_{2}\left(\Gamma_{0}(108), \chi\right)$ in terms of Eisenstein series and eta quotients. The literature on odd weight modular form spaces is not as rich as for even weight modular form spaces. In the first section, in order to give an example of constructing bases for odd weight modular forms, we give bases for $M_{3}\left(\Gamma_{0}(24), \chi_{-3}\right), M_{3}\left(\Gamma_{0}(24), \chi_{-4}\right)$, $M_{3}\left(\Gamma_{0}(24), \chi_{-8}\right)$ and $M_{3}\left(\Gamma_{0}(24), \chi_{-24}\right)$.

As discussed earlier it is not possible to give a family of eta quotients which will constitute a basis for $S_{2}\left(\Gamma_{0}\left(4 \cdot 3^{i}\right), \chi\right)$ for all $i \geq 1$. In the second section we choose $i=3$ and give bases for $M_{2}\left(\Gamma_{0}(108)\right)$ and $M_{2}\left(\Gamma_{0}(108), \chi_{12}\right)$. We choose $i=3$ for presentation purposes. We will use the results of this chapter in Chapter 10. In this chapter we denote bases of $E_{k}\left(\Gamma_{0}(N), \chi\right)$ and $S_{k}\left(\Gamma_{0}(N), \chi\right)$ by the set $E(k, N, \chi)$ and the ordered set $S(k, N, \chi)$, respectively. And $S(k, N, \chi)_{i}$ denotes the $i^{\text {th }}$ eta quotient in the ordered set 
$S(k, N, \chi)$.

\subsection{Bases for $M_{3}\left(\Gamma_{0}(24), \chi\right)$}

Theorem 9.1.1. (i) The set of Eisenstein series

$$
E\left(3,24, \chi_{-3}\right)=\left\{E_{3, \chi_{-3}, \chi_{1}}(d z), E_{3, \chi_{1}, \chi_{-3}}(d z) \mid d=1,2,4,8\right\}
$$

constitute a basis for $E_{3}\left(\Gamma_{0}(24), \chi_{-3}\right)$.

(ii) The ordered set of eta quotients

$$
\begin{aligned}
S\left(3,24, \chi_{-3}\right)= & \left\{\eta_{24}[0,3,0,-4,-5,2,16,-6](z), \eta_{24}[1,-1,-3,1,7,0,1,0](z),\right. \\
& \left.\eta_{24}[0,2,0,-1,-2,0,7,0](z), \eta_{24}[0,1,0,2,1,-2,-2,6](z)\right\}
\end{aligned}
$$

constitute a basis for $S_{3}\left(\Gamma_{0}(24), \chi_{-3}\right)$.

(iii) The set $E\left(3,24, \chi_{-3}\right) \cup S\left(3,24, \chi_{-3}\right)$ constitute a basis for $M_{3}\left(\Gamma_{0}(24), \chi_{-3}\right)$.

Proof. Let $\varepsilon$ and $\psi$ be primitive characters with conductors $R$ and $L$, respectively. By Theorem 3.2.2, the set of Eisenstein series

$$
\left\{E_{2, \varepsilon, \psi}(t z)\left|\varepsilon \psi=\chi_{-3}, R L t\right| 24\right\}
$$

gives a basis for $E_{3}\left(\Gamma_{0}(24), \chi_{-3}\right)$. All (primitive) characters with conductors dividing 24 and their values at numbers coprime to 24 are given in the following table, where $C$ denotes conductor of the corresponding character. 


\begin{tabular}{lrrrrrrrrr}
\hline \hline & $C$ & 1 & 5 & 7 & 11 & 13 & 17 & 19 & 23 \\
\hline \hline$\chi_{1}$ & 1 & 1 & 1 & 1 & 1 & 1 & 1 & 1 & 1 \\
$\chi_{-24}$ & 24 & 1 & 1 & 1 & 1 & -1 & -1 & -1 & -1 \\
$\chi_{-4}$ & 4 & 1 & 1 & -1 & -1 & 1 & 1 & -1 & -1 \\
$\chi_{24}$ & 24 & 1 & 1 & -1 & -1 & -1 & -1 & 1 & 1 \\
$\chi_{8}$ & 8 & 1 & -1 & 1 & -1 & -1 & 1 & -1 & 1 \\
$\chi_{-3}$ & 3 & 1 & -1 & 1 & -1 & 1 & -1 & 1 & -1 \\
$\chi_{-8}$ & 8 & 1 & -1 & -1 & 1 & -1 & 1 & 1 & -1 \\
$\chi_{12}$ & 12 & 1 & -1 & -1 & 1 & 1 & -1 & -1 & 1 \\
\hline
\end{tabular}

From the table we calculate

$$
\begin{aligned}
& \chi_{1} \chi_{-3}=\chi_{-3}, \quad \chi_{-3} \chi_{1}=\chi_{-3} \text { with } R L=3 \mid 24, \\
& \chi_{8} \chi_{-24}=\chi_{-3}, \quad \chi_{-24} \chi_{8}=\chi_{-3} \text { with } R L=192 \nmid 24, \\
& \chi_{-8} \chi_{24}=\chi_{-3}, \quad \chi_{24} \chi_{-8}=\chi_{-3} \text { with } R L=192 \nmid 24, \\
& \chi_{-4} \chi_{12}=\chi_{-3}, \quad \chi_{12} \chi_{-4}=\chi_{-3} \text { with } R L=48 \nmid 24 .
\end{aligned}
$$

That is $\left\{E_{2, \varepsilon, \psi}(t z)\left|\varepsilon \psi=\chi_{-3}, R L t\right| 24\right\}=E\left(3,24, \chi_{-3}\right)$. Thus we deduce that the set of Eisenstein series given by $E\left(3,24, \chi_{-3}\right)$ constitute a basis for $E_{3}\left(\Gamma_{0}(24), \chi_{-3}\right)$

Now we prove assertion (ii) of the theorem. From [70, Section 6.3] we 
obtain

$$
\operatorname{dim}\left(S_{3}\left(\Gamma_{0}(24), \chi_{-3}\right)\right)=4
$$

By Theorem 2.2.3 we have

$$
R\left(\Gamma_{0}(24)\right)=\left\{1, \frac{1}{2}, \frac{1}{3}, \frac{1}{4}, \frac{1}{6}, \frac{1}{8}, \frac{1}{12}, \frac{1}{24}\right\}
$$

Let $f(z)=\eta_{24}[0,3,0,-4,-5,2,16,-6](z)$. By (4.1.4), for $r \in R\left(\Gamma_{0}(24)\right)$ we have

$$
v_{r}(f(z))= \begin{cases}5, & \text { if } r=\frac{1}{12} \\ 1, & \text { otherwise }\end{cases}
$$

So, by Theorem 4.2.6 and 4.2.13), $f(z) \in S_{3}\left(\Gamma_{0}(24), \chi_{-3}\right)$. Similarly we show that for all eta quotients $f(z) \in S\left(3,24, \chi_{-3}\right)$, we have $f(z) \in S_{3}\left(\Gamma_{0}(24), \chi_{-3}\right)$. The orders of all the eta quotients in $S\left(3,24, \chi_{-3}\right)$ at the cusp $\frac{1}{24}$ (or equivalently at $\infty)$ are different. Thus eta quotients given in $S\left(3,24, \chi_{-3}\right)$ are linearly independent, which completes the proof of part (ii).

Part (iii) of theorem follows from (1.0.1).

The rest of the theorems in this chapter can be proven similarly.

Theorem 9.1.2. (i) The set of Eisenstein series

$$
E\left(3,24, \chi_{-4}\right)=\left\{E_{3, \chi_{-4}, \chi_{1}}(d z), E_{3, \chi_{1}, \chi_{-4}}(d z) \mid d=1,2,3,6\right\}
$$


constitute a basis for $E_{3}\left(\Gamma_{0}(24), \chi_{-4}\right)$.

(ii) The ordered set of eta quotients

$$
\begin{aligned}
S\left(3,24, \chi_{-4}\right)= & \left\{\eta_{24}[1,-1,-3,0,7,2,2,-2](z), \eta_{24}[0,2,0,-2,-2,2,8,-2](z),\right. \\
& \left.\eta_{24}[0,0,0,4,0,-2,2,2](z), \eta_{24}[0,1,0,1,1,0-1,4](z)\right\}
\end{aligned}
$$

constitute a basis for $S_{3}\left(\Gamma_{0}(24), \chi_{-4}\right)$.

(iii) The set $E\left(3,24, \chi_{-4}\right) \cup S\left(3,24, \chi_{-4}\right)$ constitute a basis for $M_{3}\left(\Gamma_{0}(24), \chi_{-4}\right)$.

Theorem 9.1.3. (i) The set of Eisenstein series

$$
E\left(3,24, \chi_{-8}\right)=\left\{E_{3, \chi_{-8}, \chi_{1}}(d z), E_{3, \chi_{1}, \chi_{-8}}(d z) \mid d=1,3\right\}
$$

constitute a basis for $E_{3}\left(\Gamma_{0}(24), \chi_{-8}\right)$.

(ii) The ordered set of eta quotients

$$
\begin{aligned}
S\left(3,24, \chi_{-8}\right)= & \left\{\eta_{24}[2,-2,-4,-2,7,2,7,-4](z), \eta_{24}[1,1,-1,-4,-2,2,13,-4](z),\right. \\
& \eta_{24}[2,-3,-4,1,10,0,-2,2](z), \eta_{24}[1,0,-1,-1,1,0,4,2](z), \\
& \left.\eta_{24}[0,1,2,0,-2,-1,1,5](z), \eta_{24}[1,-1,-1,2,4,-2,-5,8](z)\right\}
\end{aligned}
$$

constitute a basis for $S_{3}\left(\Gamma_{0}(24), \chi_{-8}\right)$.

(iii) The set $E\left(3,24, \chi_{-8}\right) \cup S\left(3,24, \chi_{-8}\right)$ constitute a basis for $M_{3}\left(\Gamma_{0}(24), \chi_{-8}\right)$. 
Theorem 9.1.4. (i) The set of Eisenstein series

$$
E\left(3,24, \chi_{-24}\right)=\left\{E_{3, \chi_{-24}, \chi_{1}}(z), E_{3, \chi_{1}, \chi_{-24}}(z), E_{3, \chi_{-3}, \chi_{8}}(z), E_{3, \chi_{8}, \chi_{-3}}(z)\right\}
$$

constitute a basis for $E_{3}\left(\Gamma_{0}(24), \chi_{-24}\right)$.

(ii) The ordered set of eta quotients

$$
\begin{aligned}
S\left(3,24, \chi_{-24}\right)= & \left\{\eta_{24}[1,1,-1,-5,-2,4,14,-6](z), \eta_{24}[2,-3,-4,0,10,2,-1,0](z)\right. \\
& \eta_{24}[1,0,-1,-2,1,2,5,0](z), \eta_{24}[1,-2,-1,4,3,-2,-1,4](z), \\
& \left.\eta_{24}[1,-1,-1,1,4,0,-4,6](z), \eta_{24}[-1,4,1,0,-1,-2,-3,8](z)\right\}
\end{aligned}
$$

constitute a basis for $S_{3}\left(\Gamma_{0}(24), \chi_{-24}\right)$.

(iii) The set $E\left(3,24, \chi_{-24}\right) \cup S\left(3,24, \chi_{-24}\right)$ constitute a basis for $M_{3}\left(\Gamma_{0}(24), \chi_{-24}\right)$.

\subsection{Bases for $M_{2}\left(\Gamma_{0}(108), \chi\right)$}

Theorem 9.2.1. (i) The set of Eisenstein series

$$
E\left(2,108, \chi_{1}\right)=\left\{L_{t}|1<t| 108\right\} \cup\left\{E_{2, \chi_{-3}, \chi_{-3}}(d z)|1 \leq d| 12\right\}
$$

constitute a basis for $E_{2}\left(\Gamma_{0}(108)\right)$.

(ii) The ordered set of eta quotients

$$
S\left(2,108, \chi_{1}\right)=\left\{\eta_{108}[0,0,0,0,1,2,0,-4,-6,2,15,-6](z)\right.
$$




$$
\begin{aligned}
& \eta_{108}[0,0,1,0,1,0,-1,-1,-1,3,2,0](z), \\
& \eta_{108}[0,0,2,0,0,0,0,0,-2,0,4,0](z), \\
& \eta_{108}[1,0,0,-1,-1,-1,3,3,0,0,0,0](z), \\
& \eta_{108}[1,-1,1,1,0,-1,0,3,-1,-1,2,0](z), \\
& \eta_{108}[2,-1,0,0,0,-2,0,5,0,0,0,0](z), \\
& \eta_{108}[2,-1,0,0,1,1,0,0,-1,0,2,0](z), \\
& \eta_{108}[3,-1,-1,-1,0,0,3,1,0,0,0,0](z), \\
& \eta_{108}[4,-2,-1,0,1,-1,0,3,0,0,0,0](z), \\
& \left.\eta_{108}[6,-3,-2,0,2,0,0,1,0,0,0,0](z)\right\}
\end{aligned}
$$

constitute a basis for $S_{2}\left(\Gamma_{0}(108)\right)$.

(iii) The set $E\left(2,108, \chi_{1}\right) \cup S\left(2,108, \chi_{1}\right)$ constitute a basis for $M_{2}\left(\Gamma_{0}(108)\right)$.

Theorem 9.2.2. (i) The set of Eisenstein series

$E\left(2,108, \chi_{12}\right)=\left\{E_{2, \chi_{-3}, \chi_{-4}}(d z), E_{2, \chi_{-4}, \chi_{-3}}(d z), E_{2, \chi_{12}, \chi_{1}}(d z), E_{2, \chi_{1}, \chi_{12}}(d z)|1 \leq d| 9\right\}$

constitute a basis for $E_{2}\left(\Gamma_{0}(108), \chi_{12}\right)$.

(ii) The ordered set of eta quotients

$$
\begin{aligned}
S\left(2,108, \chi_{12}\right)= & \left\{\eta_{108}[0,0,1,0,-1,-1,0,2,-2,2,5,-2](z),\right. \\
& \eta_{108}[0,0,2,0,-2,-1,1,3,-3,-1,7,-2](z), \\
& \eta_{108}[0,0,3,0,-2,-2,0,3,-1,2,3,-2](z),
\end{aligned}
$$




$$
\begin{aligned}
& \eta_{108}[1,0,0,-1,-1,1,3,-2,-2,2,5,-2](z), \\
& \eta_{108}[1,0,1,-1,-2,0,4,0,0,1,0,0](z), \\
& \eta_{108}[1,-1,2,1,0,0,-1,-2,-1,4,3,-2](z), \\
& \eta_{108}[2,-1,0,0,0,0,0,0,-2,2,5,-2](z), \\
& \eta_{108}[2,-1,1,0,-1,-1,1,2,0,1,0,0](z), \\
& \eta_{108}[4,-4,-4,1,9,2,-3,-4,-2,2,5,-2](z), \\
& \eta_{108}[4,-4,-3,1,8,1,-2,-2,0,1,0,0](z), \\
& \eta_{108}[4,-2,-1,0,1,1,0,-2,-2,2,5,-2](z), \\
& \left.\eta_{108}[4,-2,0,0,0,0,1,0,0,1,0,0](z)\right\}
\end{aligned}
$$

constitute a basis for $S_{2}\left(\Gamma_{0}(108), \chi_{12}\right)$.

(iii) The set $E\left(2,108, \chi_{12}\right) \cup S\left(2,108, \chi_{12}\right)$ constitute a basis for $M_{2}\left(\Gamma_{0}(108), \chi_{12}\right)$.

We finish this chapter noting that in Appendix C we give a list of sets which generate various modular forms spaces. 


\section{Chapter 10}

\section{Representations by Certain Quadratic Forms}

Finding the formulas for number of representations of a number by quadratic forms is a classic study in number theory. See [31] for a classical history of this research. For contemporary accounts of the subject see [13, 14, 19, 24, 33, 43, 50, 57]. In this chapter we apply the results given in Chapter 9 to find a formula for number of representations of a number by certain quadratic forms. A couple of our results were previously known, results presented in Tables 10.1.1 10.2.2 are mostly new results.

In the first section, we find formulas for

$$
N\left(a_{1}, \ldots, a_{6} ; n\right)=\left|\left\{\left(x_{1}, \ldots, x_{6}\right) \in \mathbb{Z}^{6} \mid n=a_{1} x_{1}^{2}+\cdots+a_{6} x_{6}^{2}\right\}\right|
$$

for all $a_{i} \in\{1,2,3,6\}(1 \leq i \leq 6)$. 
In the second section, we find formulas for

$N\left(a_{1}, a_{2}, a_{3}, a_{4} ; n\right)=\left|\left\{\left(x_{1}, x_{2}, x_{3}, x_{4}\right) \in \mathbb{Z}^{4} \mid n=a_{1} x_{1}^{2}+a_{2} x_{2}^{2}+a_{3} x_{3}^{2}+a_{4} x_{4}^{2}\right\}\right|$

for all $a_{i} \in\{1,3,9,27\}(1 \leq i \leq 4)$. By 1.0 .4$)$ and Theorem 4.2.6, we know that the generating functions of 10.0.1 and 10.0.2 are modular forms, whose corresponding spaces are given by Theorems 10.1.1 and 10.2.1 below. Theorems 10.1 .3 and 10.2 .3 combined with the Tables 10.1.1 10.2.2 give the desired formulas for the numbers of representations of positive integers by the corresponding quaternary and sextenary quadratic forms.

\subsection{Sextenary Quadratic Forms}

Theorem 10.1.1. Let $l_{d} \in \mathbb{N}_{0}(d \mid 6)$ and $l_{1}+l_{2}+l_{3}+l_{6}=6$. Then we have

$$
\prod_{d \mid 6} \varphi^{l_{d}}(d z) \in \begin{cases}M_{3}\left(\Gamma_{0}(24), \chi_{-4}\right) & \text { if } l_{1}+l_{3} \equiv 0(\bmod 2) \\ & \text { and } l_{3}+l_{6} \equiv 0(\bmod 2), \\ M_{3}\left(\Gamma_{0}(24), \chi_{-3}\right) & \text { if } l_{1}+l_{3} \equiv 0(\bmod 2) \\ & \text { and } l_{3}+l_{6} \equiv 1(\bmod 2), \\ M_{3}\left(\Gamma_{0}(24), \chi_{-8}\right) & \text { if } l_{1}+l_{3} \equiv 1(\bmod 2) \\ & \text { and } l_{3}+l_{6} \equiv 0(\bmod 2), \\ M_{3}\left(\Gamma_{0}(24), \chi_{-24}\right) & \text { if } l_{1}+l_{3} \equiv 1(\bmod 2) \\ & \text { and } l_{3}+l_{6} \equiv 1(\bmod 2) .\end{cases}
$$


Proof. Let $l_{d} \in \mathbb{N}_{0}(d \mid 6)$ and $l_{1}+l_{2}+l_{3}+l_{6}=6$, then we have

$$
\begin{aligned}
& \prod_{d \mid 6} \varphi^{l_{d}}(d z)=\prod_{d \mid 6} \frac{\eta^{5 l_{d}}(2 d z)}{\eta^{2 l_{d}(d z) \eta^{2 l_{d}}(4 d z)}} \\
& =\eta_{24}\left[-2 l_{1}, 5 l_{1}-2 l_{2},-2 l_{3},-2 l_{1}+5 l_{2}, 5 l_{3}-2 l_{6},-2 l_{2},-2 l_{3}+5 l_{6},-2 l_{6}\right](z) \text {. }
\end{aligned}
$$

Then we have

$$
\begin{aligned}
v_{1 / c}\left(\prod_{d \mid 6} \varphi^{l_{d}}(d z)\right) & =0 \text { for } c=1,3,8,24, \\
v_{1 / 2}\left(\prod_{d \mid 6} \varphi^{l_{d}}(d z)\right) & =\frac{3}{2} l_{1}+\frac{1}{2} l_{3} \geq 0 \\
v_{1 / 4}\left(\prod_{d \mid 6} \varphi^{l_{d}}(d z)\right) & =\frac{3}{2} l_{2}+\frac{1}{2} l_{6} \geq 0 \\
v_{1 / 6}\left(\prod_{d \mid 6} \varphi^{l_{d}}(d z)\right) & =\frac{1}{2} l_{1}+\frac{3}{2} l_{3} \geq 0 \\
\left(\prod_{d / 12} \varphi^{l_{d}}(d z)\right) & =\frac{1}{2} l_{2}+\frac{3}{2} l_{6} \geq 0
\end{aligned}
$$

So by Theorem 4.2.6. $\prod_{d \mid 6} \varphi^{l_{d}}(d z)$ is in $M_{3}\left(\Gamma_{0}(24), \chi\right)$, where, by appealing to 
4.2.13, we have

$$
\chi= \begin{cases}\chi_{-4} & \text { if } l_{1}+l_{3} \text { is even and } l_{3}+l_{6} \text { is even, } \\ \chi_{-3} & \text { if } l_{1}+l_{3} \text { is even and } l_{3}+l_{6} \text { is odd } \\ \chi_{-8} & \text { if } l_{1}+l_{3} \text { is odd and } l_{3}+l_{6} \text { is even, } \\ \chi_{-24} & \text { if } l_{1}+l_{3} \text { is odd and } l_{3}+l_{6} \text { is odd }\end{cases}
$$

Appealing to 1.0.1 and Theorem 10.1.1 we have the following theorem.

Theorem 10.1.2. Let $l_{d} \in \mathbb{N}_{0}(d \mid 6)$ and $l_{1}+l_{2}+l_{3}+l_{6}=6$. Then we have

$$
\prod_{d \mid 6} \varphi^{l_{d}}(d z)
$$




$$
=\left\{\begin{array}{c}
\sum_{t \mid 6} a_{t} E_{3, \chi_{-4}, \chi_{1}}(t z)+\sum_{t \mid 6} b_{t} E_{3, \chi_{1}, \chi_{-4}}(t z)+\sum_{j=1}^{4} c_{j} S\left(3,24, \chi_{-4}\right)_{j} \\
\text { if } l_{1}+l_{3} \equiv 0(\bmod 2) \text { and } l_{3}+l_{6} \equiv 0(\bmod 2), \\
\sum_{t \mid 8} d_{t} E_{3, \chi_{-3}, \chi_{1}}(t z)+\sum_{t \mid 8} e_{t} E_{3, \chi_{1}, \chi_{-3}}(t z)+\sum_{j=1}^{4} f_{j} S\left(3,24, \chi_{-3}\right)_{j} \\
\text { if } l_{1}+l_{3} \equiv 0(\bmod 2) \text { and } l_{3}+l_{6} \equiv 1(\bmod 2), \\
\sum_{t \mid 3} g_{t} E_{3, \chi_{-8}, \chi_{1}}(t z)+\sum_{t \mid 3} h_{t} E_{3, \chi_{1}, \chi_{-8}}(t z)+\sum_{j=1}^{6} k_{j} S\left(3,24, \chi_{-8}\right)_{j} \\
\text { if } l_{1}+l_{3} \equiv 1(\bmod 2) \text { and } l_{3}+l_{6} \equiv 0(\bmod 2), \\
m_{1} E_{3, \chi_{-24}, \chi_{1}}(z)+m_{2} E_{3, \chi_{1}, \chi_{-24}}(z)+m_{3} E_{3, \chi_{-3}, \chi_{8}}(z)+m_{4} E_{3, \chi_{8}, \chi_{-3}}(z) \\
+\sum_{j=1}^{6} n_{j} S\left(3,24, \chi_{-24}\right)_{j} \\
\text { if } l_{1}+l_{3} \equiv 1(\bmod 2) \text { and } l_{3}+l_{6} \equiv 1(\bmod 2) .
\end{array}\right.
$$

where the values $a_{t}, b_{t}, c_{j}, d_{t}, e_{t}, f_{j}, g_{t}, h_{t}, k_{j}, m_{i}, n_{j}$ are given in Tables 10.1.1-10.1.4.

The following theorem follows from Theorem 10.1.2.

Theorem 10.1.3. Let $l_{d} \in \mathbb{N}_{0}(d \mid 6)$ and $l_{1}+l_{2}+l_{3}+l_{6}=6$. Then we have $N\left(1^{l_{1}}, 2^{l_{2}}, 3^{l_{3}}, 6^{l_{6}} ; n\right)$ 


$$
=\left\{\begin{array}{c}
\sum_{t \mid 6} a_{t} \sigma_{\left(2, \chi_{-4}, \chi_{1}\right)}(n / t)+\sum_{t \mid 6} b_{t} \sigma_{\left(2, \chi_{1}, \chi_{-4}\right)}(n / t)+\sum_{j=1}^{4} c_{j}[n] S\left(3,24, \chi_{-4}\right)_{j} \\
\text { if } l_{1}+l_{3} \equiv 0(\bmod 2) \text { and } l_{3}+l_{6} \equiv 0(\bmod 2), \\
\sum_{t \mid 8} d_{t} \sigma_{\left(2, \chi_{-3}, \chi_{1}\right)}(n / t)+\sum_{t \mid 8} e_{t} \sigma_{\left(2, \chi_{1}, \chi_{-3}\right)}(n / t)+\sum_{j=1}^{4} f_{j}[n] S\left(3,24, \chi_{-3}\right)_{j} \\
\text { if } l_{1}+l_{3} \equiv 0(\bmod 2) \text { and } l_{3}+l_{6} \equiv 1(\bmod 2), \\
\sum_{t \mid 3} g_{t} \sigma_{\left(2, \chi_{-8}, \chi_{1}\right)}(n / t)+\sum_{t \mid 3} h_{t} \sigma_{\left(2, \chi_{1}, \chi_{-8}\right)}(n / t)+\sum_{j=1}^{6} k_{j}[n] S\left(3,24, \chi_{-8}\right)_{j} \\
\text { if } l_{1}+l_{3} \equiv 1(\bmod 2) \text { and } l_{3}+l_{6} \equiv 0(\bmod 2), \\
m_{1} \sigma_{\left(2, \chi_{-24}, \chi_{1}\right)}(n)+m_{2} \sigma_{\left(2, \chi_{1}, \chi_{-24}\right.}(n)+m_{3} \sigma_{\left(2, \chi_{-3}, \chi_{8}\right)}(n)+m_{4} \sigma_{\left(2, \chi_{8}, \chi_{-3}\right)}(n) \\
+\sum_{j=1}^{6} n_{j}[n] S\left(3,24, \chi_{-24}\right)_{j} \\
\text { if } l_{1}+l_{3} \equiv 1(\bmod 2) \text { and } l_{3}+l_{6} \equiv 1(\bmod 2) .
\end{array}\right.
$$

where the values $a_{t}, b_{t}, c_{j}, d_{t}, e_{t}, f_{j}, g_{t}, h_{t}, k_{j}, m_{i}, n_{j}$ are given in Tables 10.1.1-10.1.4.

We generate the following tables by comparing the first few coefficients of the Fourier series expansions of both sides in 10.1.3. 


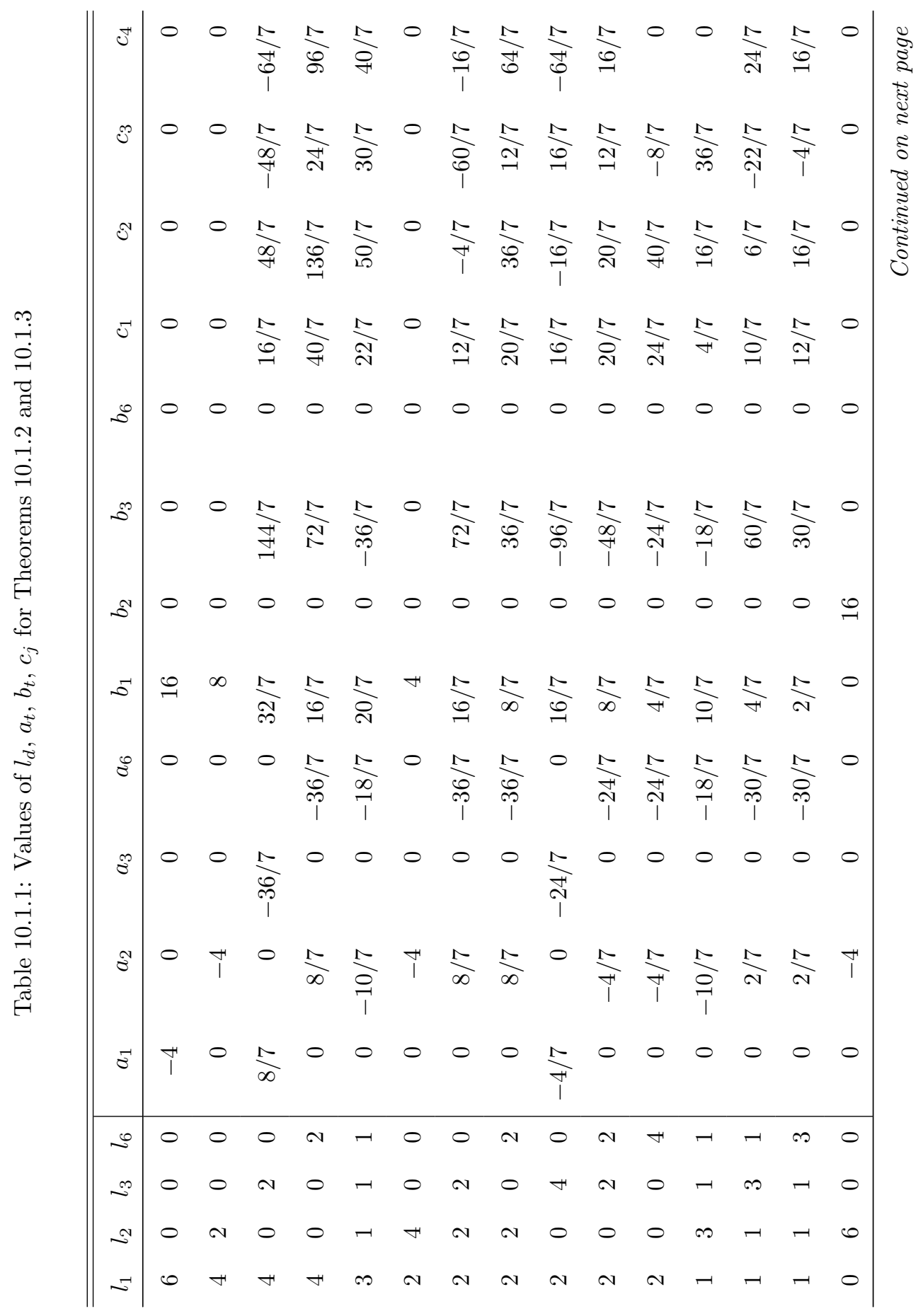




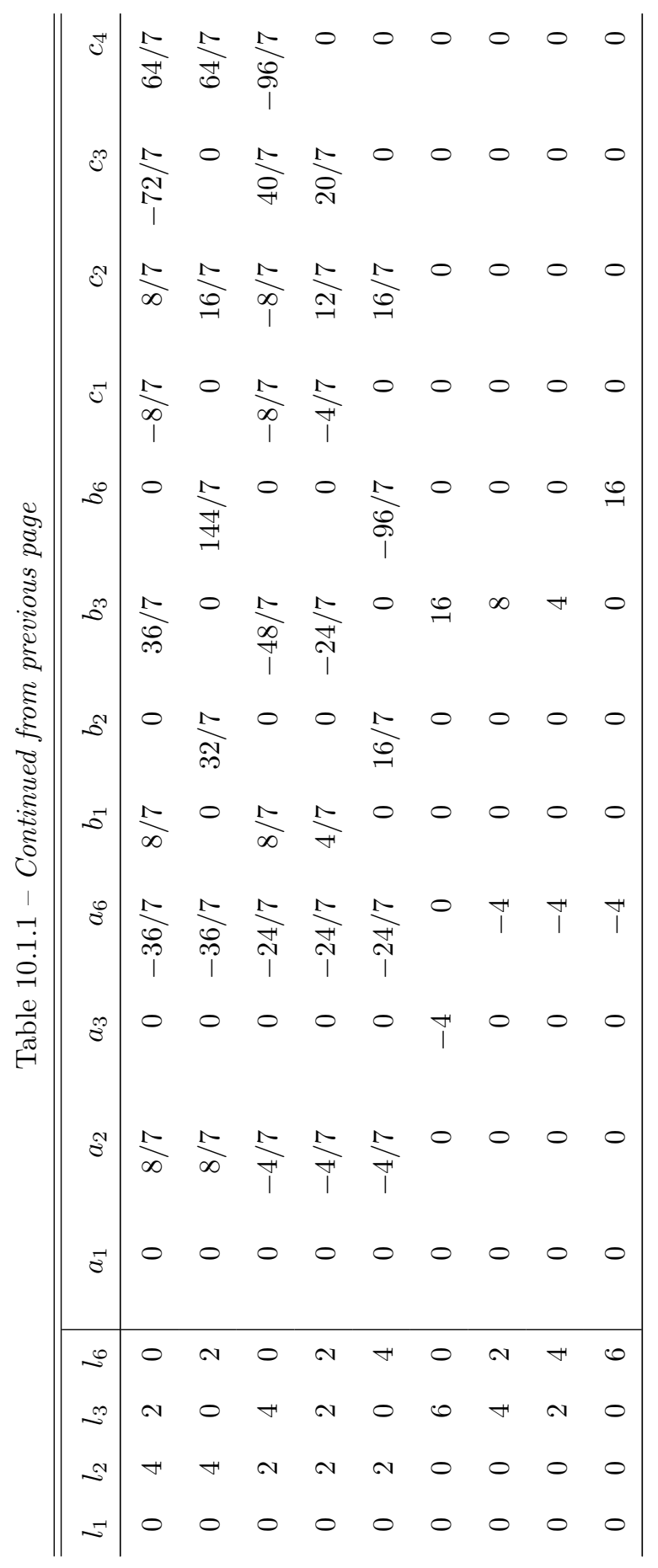




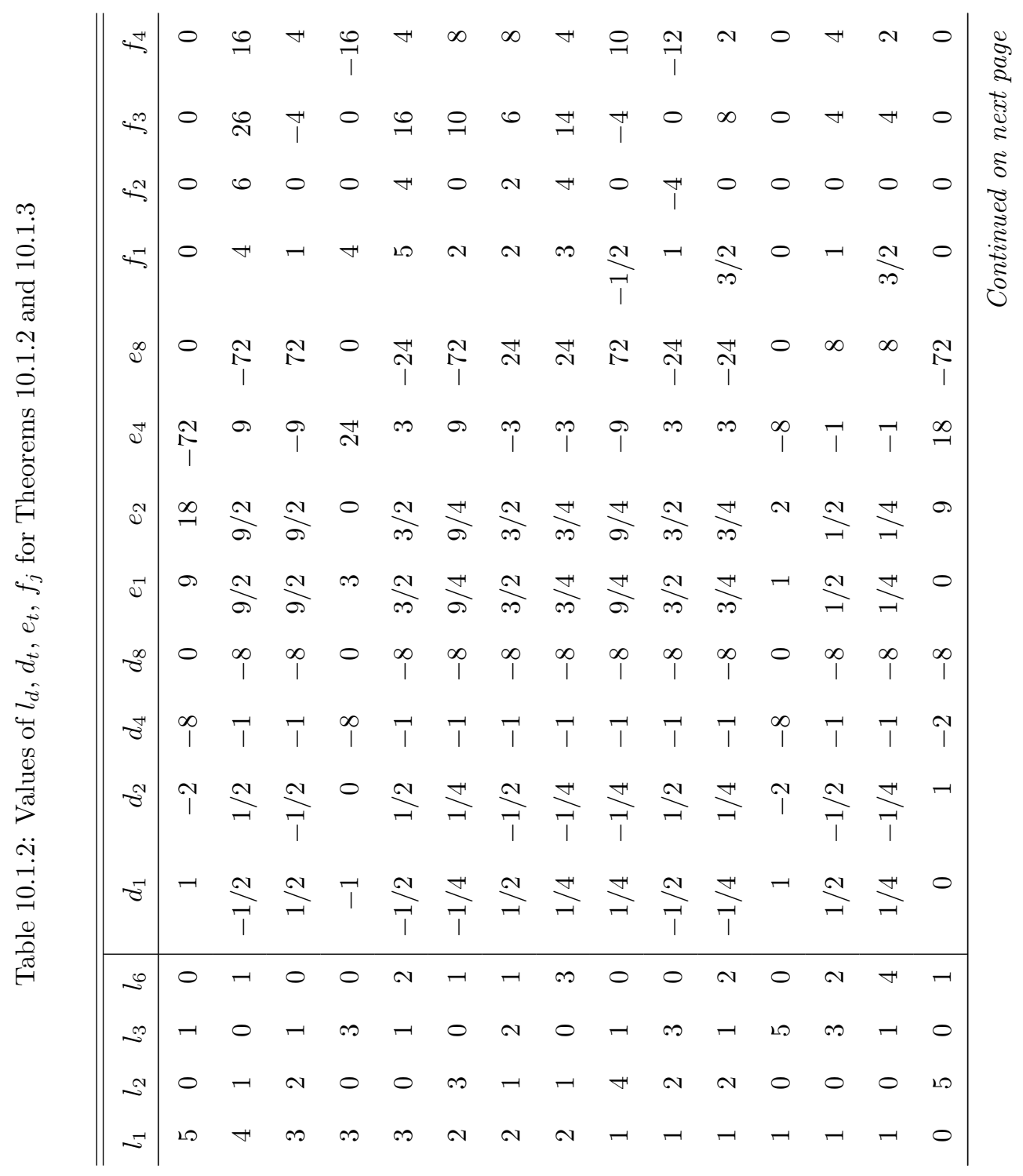




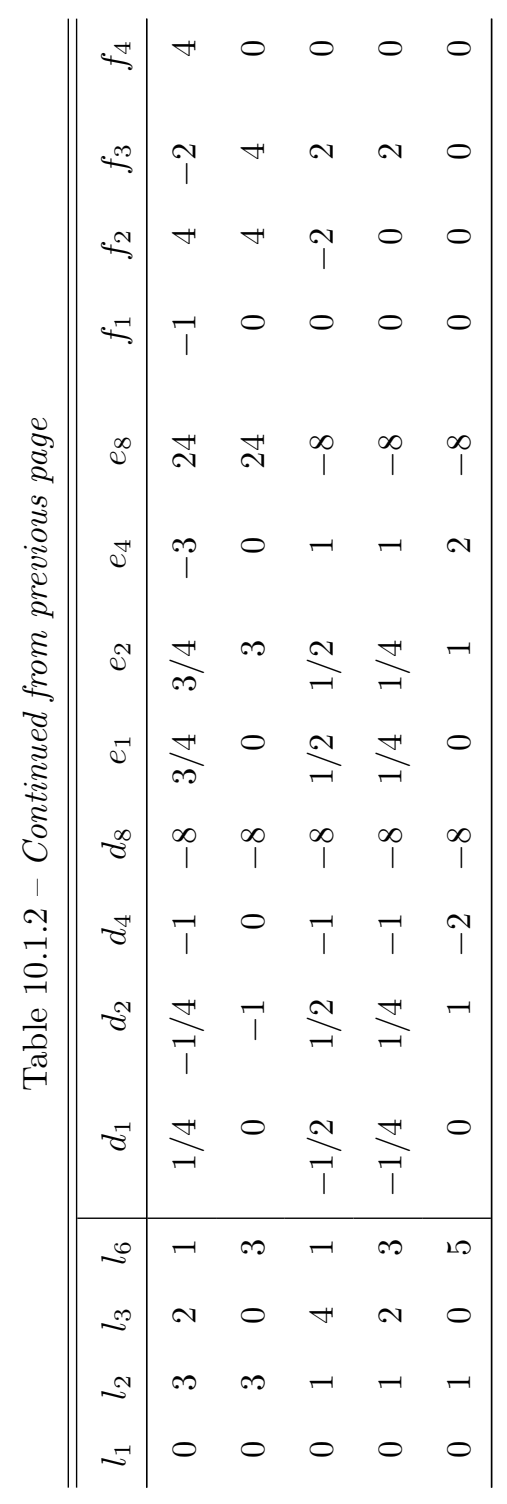




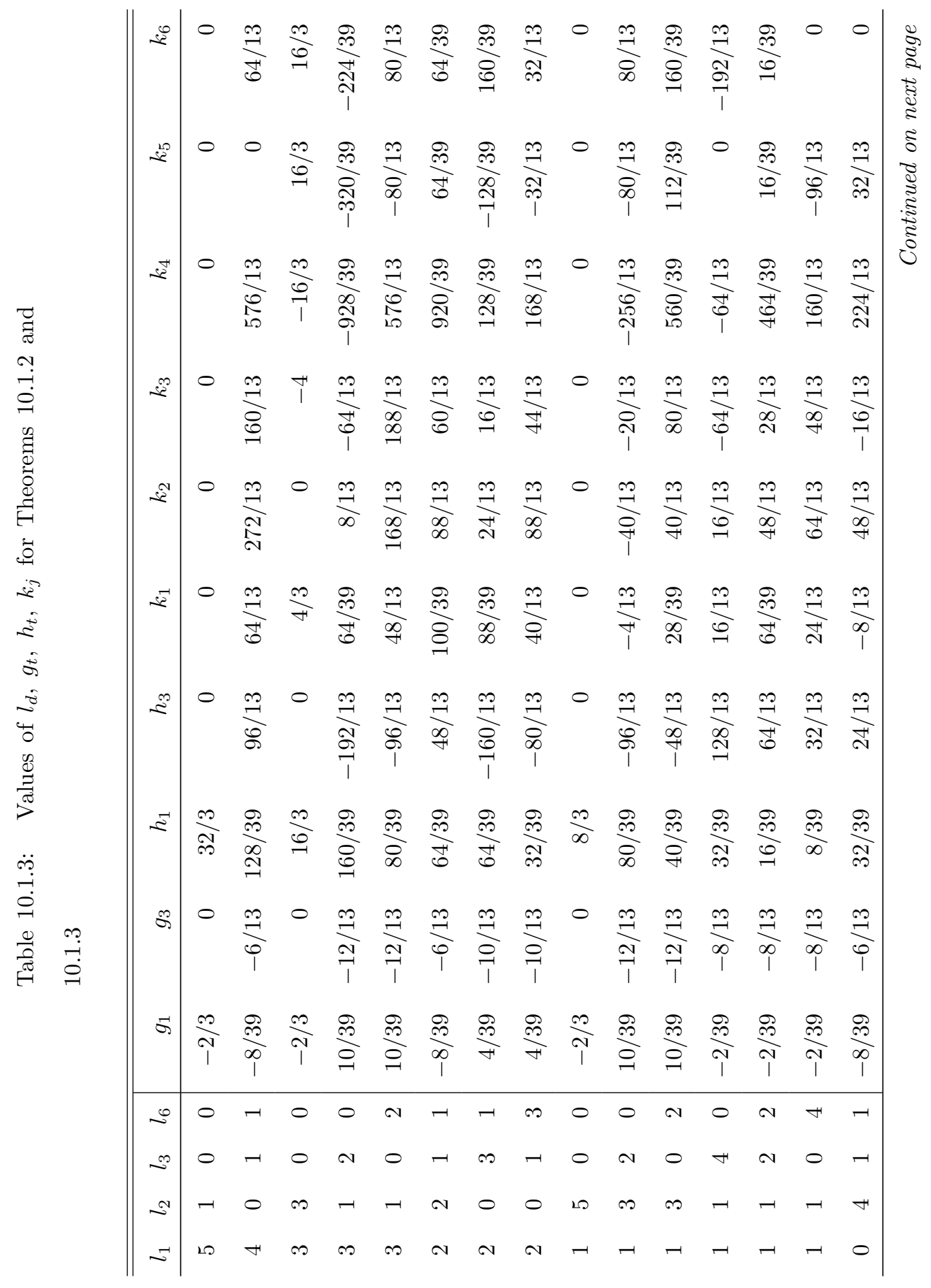




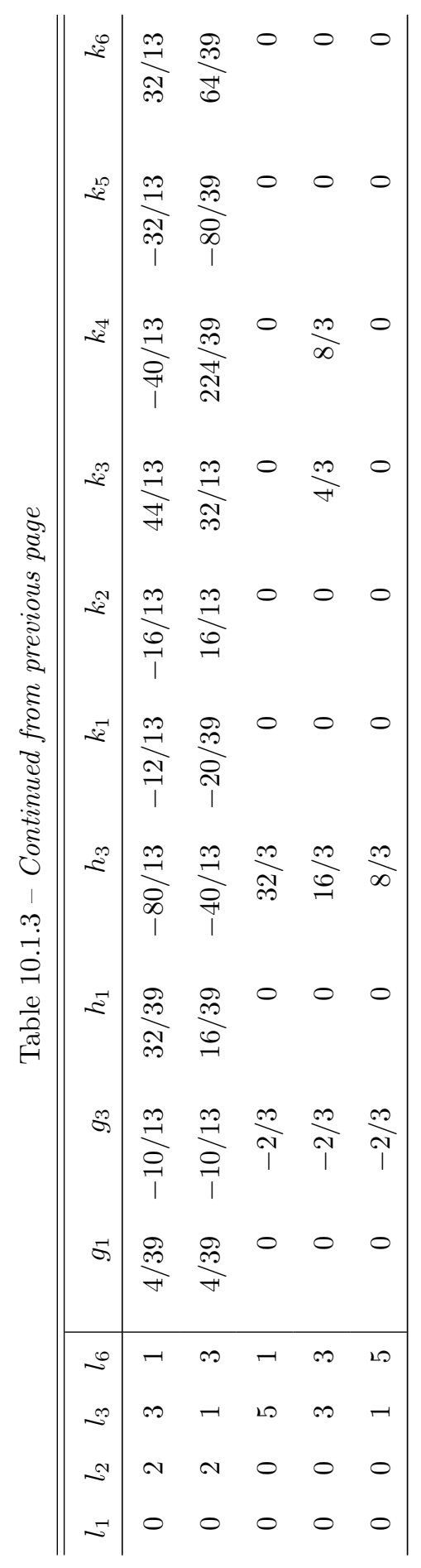




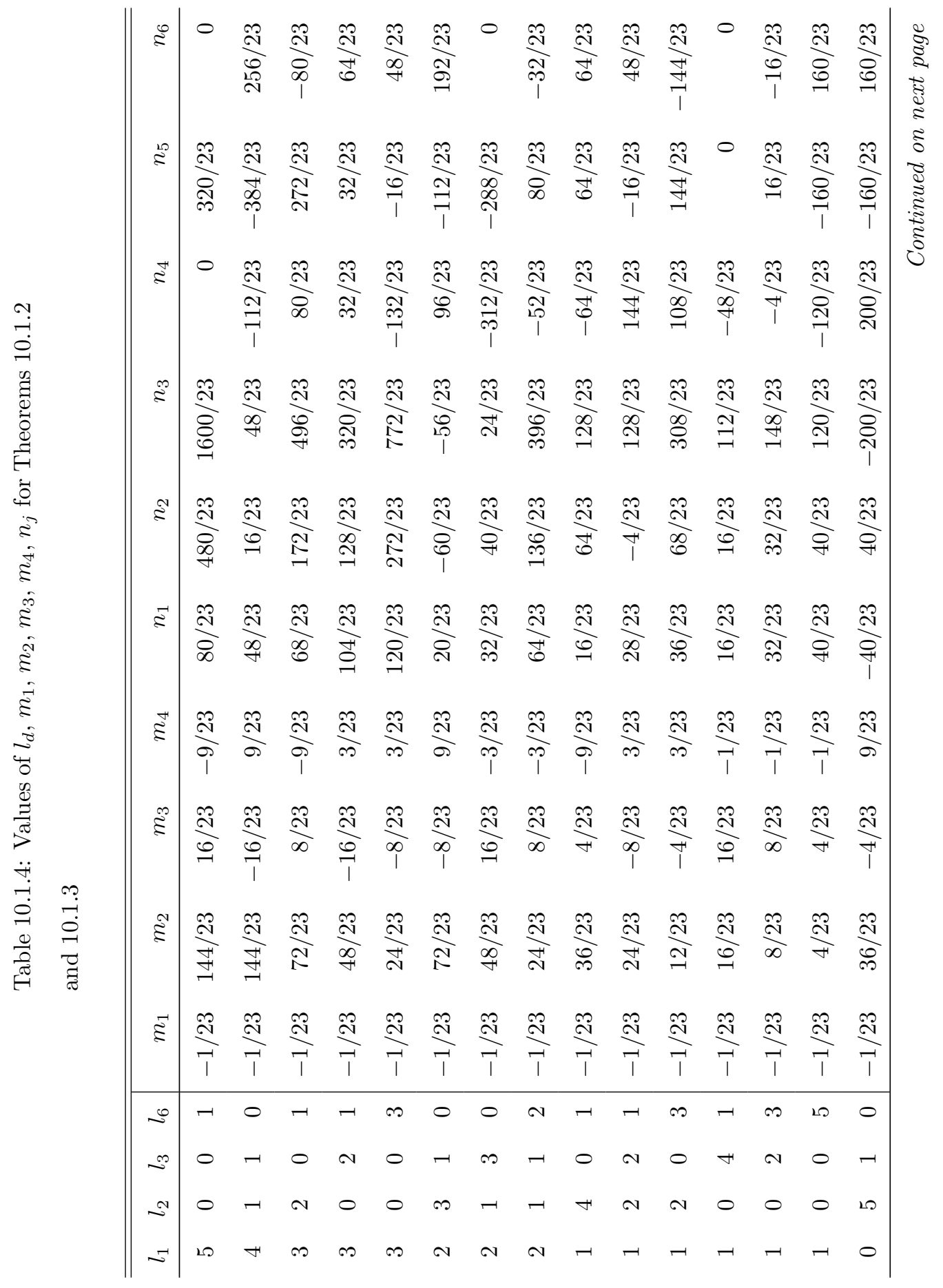




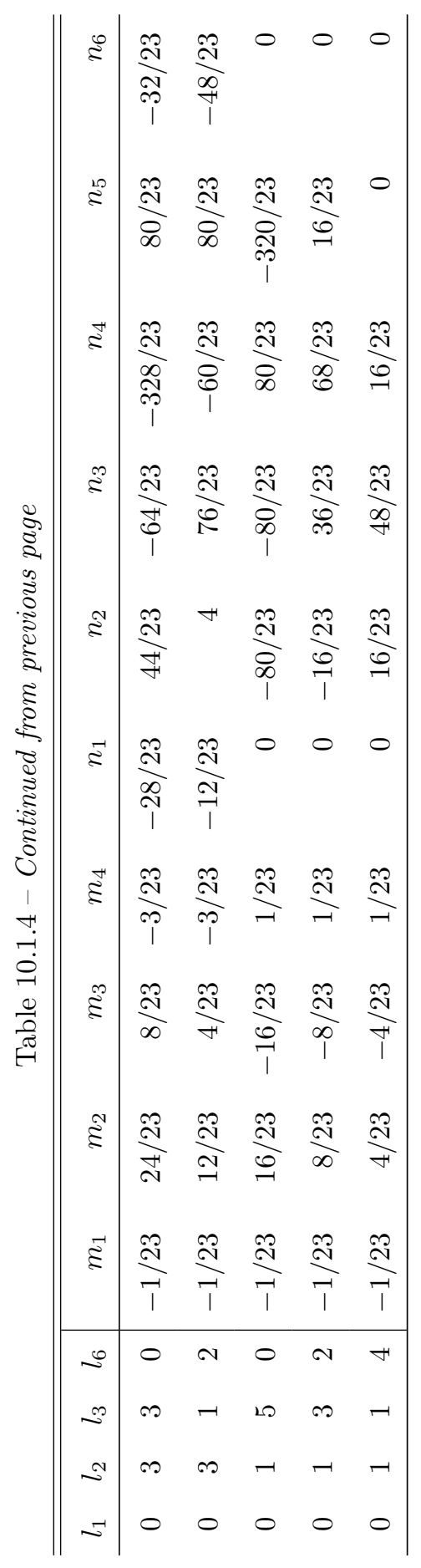




\subsection{Quaternary Quadratic Forms}

Theorem 10.2.1. Let $l_{i} \in \mathbb{N}_{0}$ for $0 \leq i \leq 3$ such that $l_{0}+l_{1}+l_{2}+l_{3}=4$.

Then we have

$$
\prod_{i=0}^{3} \varphi^{l_{i}}\left(3^{i} z\right) \in \begin{cases}M_{2}\left(\Gamma_{0}(108)\right) & \text { if } l_{1}+l_{2} \equiv 0(\bmod 2) \\ M_{2}\left(\Gamma_{0}(108), \chi_{12}\right) & \text { if } l_{1}+l_{2} \equiv 1(\bmod 2) .\end{cases}
$$

Proof. Let $l_{i} \in \mathbb{N}_{0}$ for $0 \leq i \leq 3$ such that $l_{0}+l_{1}+l_{2}+l_{3}=4$. then we have

$$
\begin{aligned}
& \prod_{i=0}^{3} \varphi^{l_{i}}\left(3^{i} z\right)=\prod_{i=0}^{3} \frac{\eta^{5 l_{i}}\left(23^{i} z\right)}{\eta^{2 l_{i}}\left(3^{i} z\right) \eta^{2 l_{i}}\left(43^{i} z\right)} \\
& =\eta_{108}\left[-2 l_{0}, 5 l_{0},-2 l_{1},-2 l_{0}, 5 l_{1},-2 l_{2},-2 l_{1}, 5 l_{2},-2 l_{3},-2 l_{2}, 5 l_{3},-2 l_{3}\right](z)
\end{aligned}
$$

Thus for $0 \leq j \leq 3$ we have

$$
\begin{aligned}
& v_{\frac{1}{3^{j}}}\left(\prod_{i=0}^{3} \varphi^{l_{i}}\left(3^{i} z\right)\right)=0, \\
& v_{\frac{1}{4 \cdot 3^{j}}}\left(\prod_{i=0}^{3} \varphi^{l_{i}}\left(3^{i} z\right)\right)=0, \\
& v_{\frac{1}{2 \cdot 3^{j}}}\left(\prod_{i=0}^{3} \varphi^{l_{i}}\left(3^{i} z\right)\right)=\frac{27}{4} \sum_{i=0}^{3} \frac{\operatorname{gcd}\left(3^{j}, 3^{i}\right)^{2}}{\operatorname{gcd}\left(3^{2 j}, 27\right) \cdot 3^{i}} \cdot l_{i} \geq 0 .
\end{aligned}
$$

So by Theorem 4.2.6. $\prod_{i=0}^{3} \varphi^{l_{i}}\left(3^{i} z\right)$ is in $M_{2}\left(\Gamma_{0}(108), \chi\right)$, where, by appealing 
to 4.2 .13 , we have

$$
\chi= \begin{cases}\chi_{1} & \text { if } l_{1}+l_{3} \equiv 0(\bmod 2) \\ \chi_{12} & \text { if } l_{1}+l_{3} \equiv 1(\bmod 2)\end{cases}
$$

Appealing to 1.0.1 and Theorem 10.2.1 we have the following theorem.

Theorem 10.2.2. Let $l_{i} \in \mathbb{N}_{0}$ for $0 \leq i \leq 3$ such that $l_{0}+l_{1}+l_{2}+l_{3}=4$.

Then we have

$$
\begin{aligned}
& \prod_{i=0}^{3} \varphi^{l_{i}}\left(3^{i} z\right) \\
& =\left\{\begin{array}{l}
\sum_{1<t \mid 108} a_{t}\left(E_{2}(z)-t E_{2}(t z)\right)+\sum_{t \mid 12} b_{t} E_{2, \chi_{-3}, \chi_{-3}}(t z)+\sum_{j=1}^{10} c_{j} S\left(2,108, \chi_{1}\right)_{j} \\
\sum_{t \mid 9} d_{t} E_{2, \chi_{-3}, \chi_{-4}}(t z)+\sum_{t \mid 9} e_{t} E_{2, \chi_{-4}, \chi_{-3}}(t z)+\sum_{t \mid 9} f_{t} E_{2, \chi_{12}, \chi_{1}}(t z) \\
+\sum_{t \mid 9} g_{t} E_{2, \chi_{1}, \chi_{12}}(t z)+\sum_{j=1}^{12} h_{j} S\left(2,108, \chi_{12}\right)_{j}
\end{array} \quad \text { if } l_{1}+l_{2} \equiv 1(\bmod 2),\right.
\end{aligned}
$$

where the values $a_{t}, b_{j}, c_{t}, d_{t}, e_{t}, f_{t}, g_{j}$ are given in Tables 10.2.1 and 10.2.2.

The following theorem follows from Theorem 10.2.2.

Theorem 10.2.3. Let $l_{i} \in \mathbb{N}_{0}$ for $0 \leq i \leq 3$, such that $l_{0}+l_{1}+l_{2}+l_{3}=4$. 
Then we have

$$
\begin{aligned}
& N\left(1^{l_{0}}, 3^{l_{1}}, 9^{l_{2}}, 27^{l_{3}} ; n\right) \\
& =\left\{\begin{array}{c}
\sum_{1<t \mid 108} a_{t}(\sigma(n)-t \sigma(n / t))+\sum_{t \mid 12} b_{t} \sigma_{1, \chi_{-3}, \chi_{-3}}(n / t)+\sum_{j=1}^{10} c_{j}[n] S\left(2,108, \chi_{1}\right)_{j} \\
\sum_{t \mid 9} d_{t} \sigma_{\left(1, \chi_{-3}, \chi_{-4}\right)}(n / t)+\sum_{t \mid 9} e_{t} \sigma_{\left(1, \chi_{-4}, \chi_{-3}\right)}(n / t)+\sum_{t \mid 9} f_{t} \sigma_{\left(1, \chi_{12}, \chi_{1}\right)}(n / t) \\
+\sum_{t \mid 9} g_{t} \sigma_{\left(1, \chi_{1}, \chi_{12}\right)}(n / t)+\sum_{j=1}^{12} h_{j}[n] S\left(2,108, \chi_{12}\right)_{j} \\
\text { if } l_{1}+l_{2} \equiv 1(\bmod 2),
\end{array}\right.
\end{aligned}
$$

where the values $a_{t}, b_{j}, c_{t}, d_{t}, e_{t}, f_{t}, g_{j}$ are given in Tables 10.2.1 and 10.2.2. 


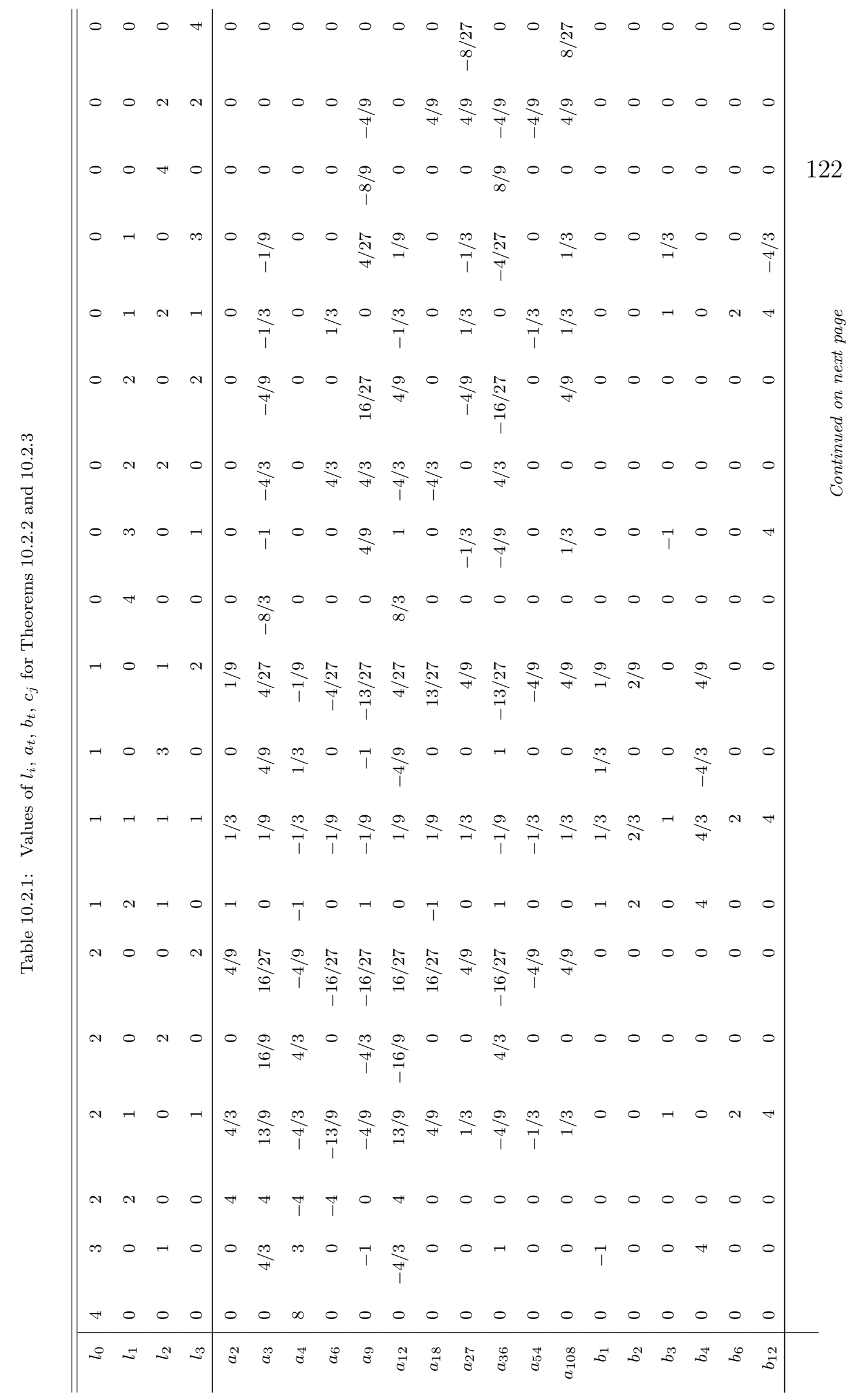




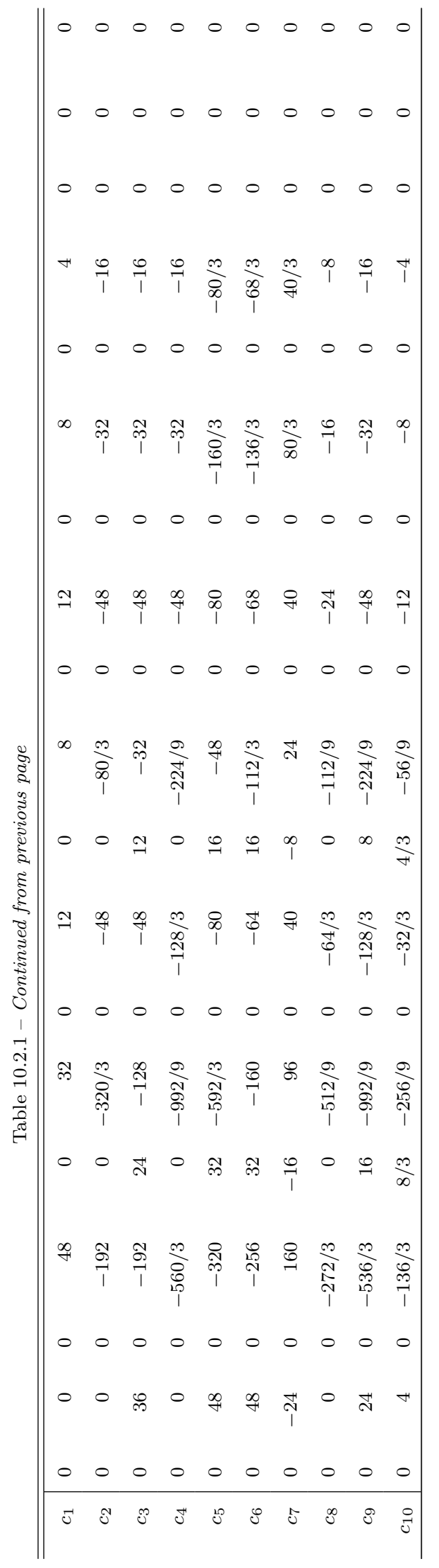




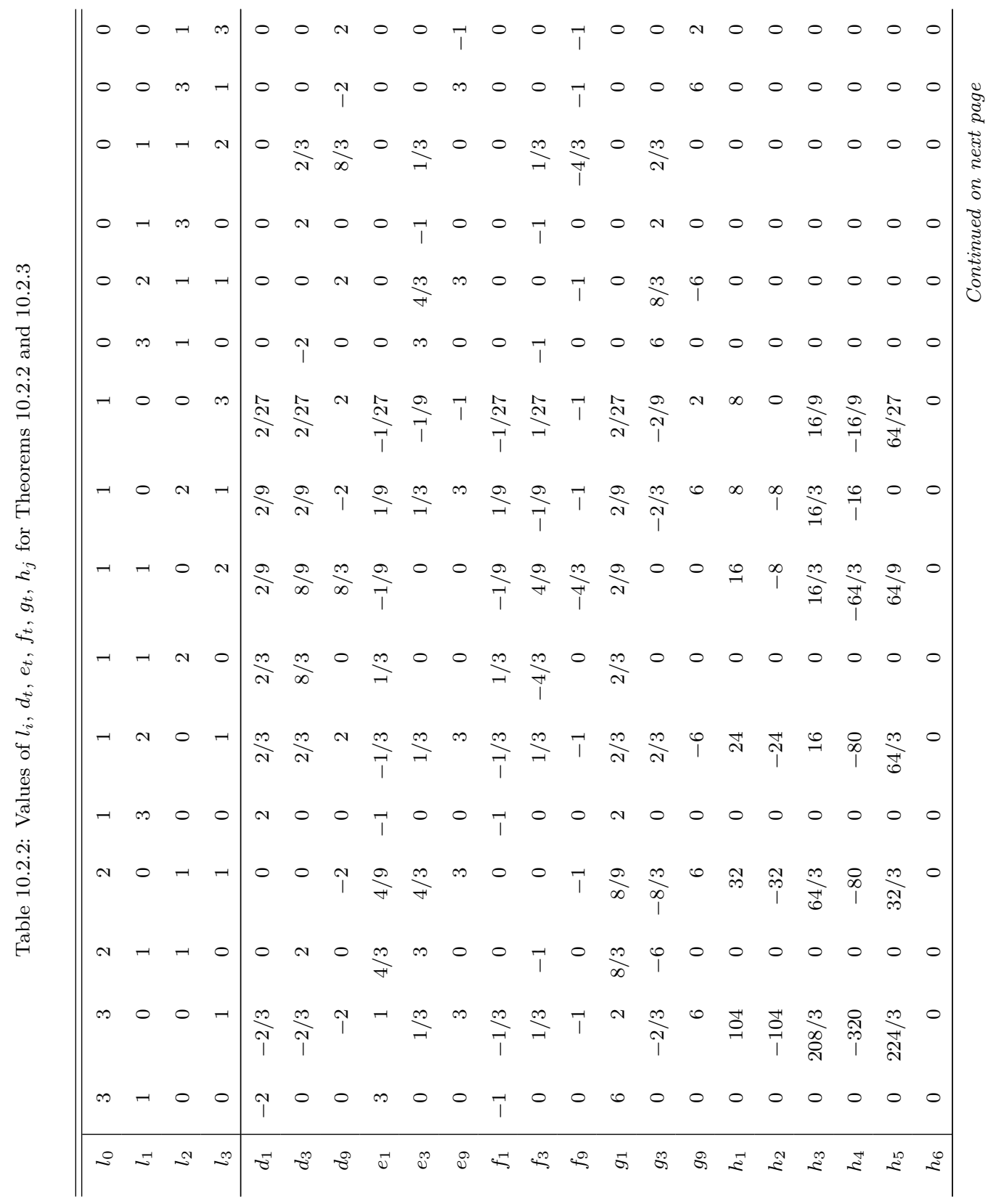




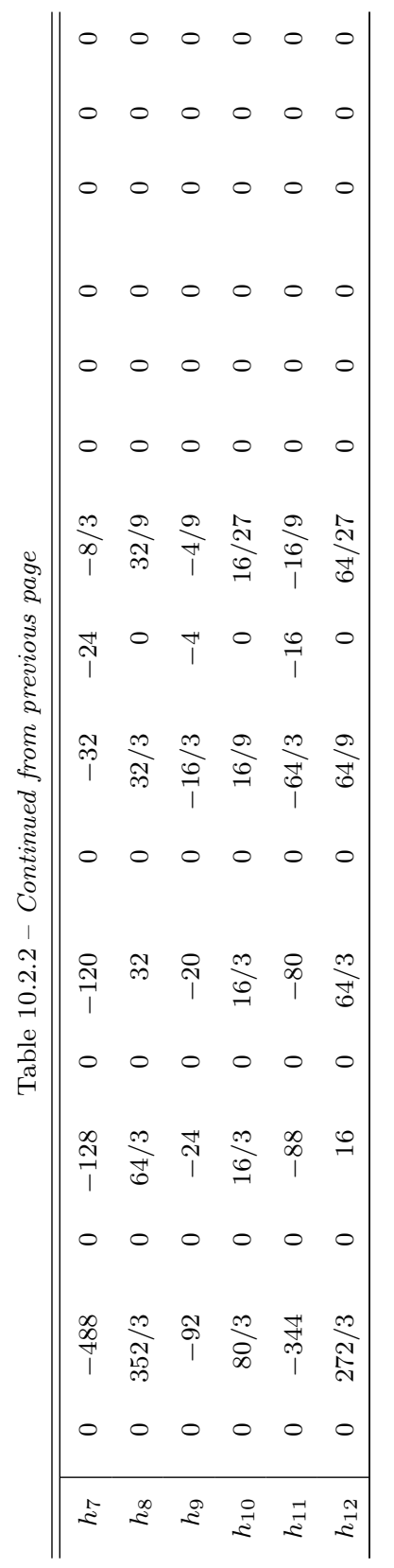




\section{Chapter 11}

\section{Fourier Coefficients of Certain Families of Eta Quotients}

Arithmetic relations between Fourier coefficients of eta quotients have been studied by S. Ahlgren, A. Berkovich, R. Chapman, S. Cooper, M. D. Hirschhorn, R. Lewis and K. S. Williams among others. (See [1, 16, 21, 25, 26, 32, 41, 54, 72] for contemporary works.) In [72], K. S. Williams gave Fourier coefficients of all eta quotients in $M_{2}\left(\Gamma_{0}(12)\right)$ explicitly. His work utilizes classical elementary methods. Inspired by his work, in [4], we gave Fourier series representations of all eta quotients in $M_{2}\left(\Gamma_{0}(12), \chi_{12}\right)$. Modular form

space $M_{2}\left(\Gamma_{0}(12), \chi_{12}\right)$ has trivial cusp subspace. Thus by Theorem 3.2 .2 all eta quotients in that space can be written as a linear combination of Eisenstein series. In the first section of this chapter, we give other examples of modular form spaces with trivial cusp form subspace, and then we give their Fourier series coefficients of eta quotients in those modular form spaces in Tables 11.1.1 11.1.4.

In the second section, we use the seventh row of Table 11.1 .4 to give a 
proof for Ramanujan's partition congruence modulo 5. In the third section, as a nod to classical studies, we give some arithmetic properties of the Fourier coefficients of the eta quotients given in the first section.

We finish the chapter by giving the graph for the number of eta quotients in $E_{2}\left(\Gamma_{0}(N)\right)$ for all $n \leq 100$.

\subsection{Fourier Coefficients of Eta Quotients}

Using [70, Section 6.3, p. 98 and p. 100] we find that

$$
\begin{aligned}
& \operatorname{dim}\left(S_{2}\left(\Gamma_{0}(8), \chi_{1}\right)\right)=0, \operatorname{dim}\left(M_{2}\left(\Gamma_{0}(8), \chi_{1}\right)\right)=3, \\
& \operatorname{dim}\left(S_{2}\left(\Gamma_{0}(8), \chi_{8}\right)\right)=0, \operatorname{dim}\left(M_{2}\left(\Gamma_{0}(8), \chi_{8}\right)\right)=2, \\
& \operatorname{dim}\left(S_{2}\left(\Gamma_{0}(9), \chi_{1}\right)\right)=0, \operatorname{dim}\left(M_{2}\left(\Gamma_{0}(9), \chi_{1}\right)\right)=3, \\
& \operatorname{dim}\left(S_{2}\left(\Gamma_{0}(10), \chi_{5}\right)\right)=0, \operatorname{dim}\left(M_{2}\left(\Gamma_{0}(10), \chi_{5}\right)\right)=4 .
\end{aligned}
$$

One can also obtain the above dimensions from the formulae in [70, Section 6.3]. Thus by (1.0.1) we have

$$
\begin{aligned}
& M_{2}\left(\Gamma_{0}(8), \chi_{1}\right)=E_{2}\left(\Gamma_{0}(8), \chi_{1}\right), \\
& M_{2}\left(\Gamma_{0}(8), \chi_{8}\right)=E_{2}\left(\Gamma_{0}(8), \chi_{8}\right), \\
& M_{2}\left(\Gamma_{0}(9), \chi_{1}\right)=E_{2}\left(\Gamma_{0}(9), \chi_{1}\right), \\
& M_{2}\left(\Gamma_{0}(10), \chi_{5}\right)=E_{2}\left(\Gamma_{0}(10), \chi_{5}\right) .
\end{aligned}
$$


It follows from Theorem 3.2 .2 that the sets of Eisenstein series

$$
\begin{aligned}
& \left\{E_{2}(z)-2 E_{2}(2 z), E_{2}(z)-4 E_{2}(4 z), E_{2}(z)-8 E_{2}(8 z)\right\}, \\
& \left\{E_{2, \chi_{1}, \chi_{8}}(z), E_{2, \chi_{8}, \chi_{1}}(z)\right\} \\
& \left\{E_{2}(z)-3 E_{2}(3 z), E_{2}(z)-9 E_{2}(9 z), E_{2, \chi_{-3}, \chi_{-3}}(z)\right\} \\
& \left\{E_{2, \chi_{1}, \chi_{5}}(z), E_{2, \chi_{5}, \chi_{1}}(z), E_{2, \chi_{1}, \chi_{5}}(2 z), E_{2, \chi_{5}, \chi_{1}}(2 z)\right\}
\end{aligned}
$$

constitute bases for $M_{2}\left(\Gamma_{0}(8), \chi_{1}\right), M_{2}\left(\Gamma_{0}(8), \chi_{8}\right), M_{2}\left(\Gamma_{0}(8), \chi_{1}\right), M_{2}\left(\Gamma_{0}(10), \chi_{5}\right)$, respectively. We note that the Eisenstein series $E_{2}(z), E_{2, \chi_{1}, \chi_{8}}(z), E_{2, \chi_{8}, \chi_{1}}(z)$, $E_{2, \chi_{-3}, \chi_{-3}}(z), E_{2, \chi_{1}, \chi_{5}}(z)$ and $E_{2, \chi_{5}, \chi_{1}}(z)$ are defined in 3.2 .2$)$ as

$$
\begin{aligned}
& E_{2}(z)=-\frac{1}{24}+\sum_{n=1}^{\infty} \sigma(n) q^{n} \\
& E_{2, \chi_{8}, \chi_{1}}(z)=-\frac{1}{2}+\sum_{n=1}^{\infty} \sigma_{\left(1, \chi_{8}, \chi_{1}\right)}(n) q^{n} \\
& E_{2, \chi_{1}, \chi_{8}}(z)=\sum_{n=1}^{\infty} \sigma_{\left(1, \chi_{1}, \chi_{8}\right)}(n) q^{n} \\
& E_{2, \chi_{-3}, \chi_{-3}}(z)=\sum_{n=1}^{\infty} \sigma_{\left(1, \chi_{-3}, \chi_{-3}\right)}(n) q^{n} \\
& E_{2, \chi_{5}, \chi_{1}}(z)=-\frac{1}{5}+\sum_{n=1}^{\infty} \sigma_{\left(1, \chi_{5}, \chi_{1}\right)}(n) q^{n} \\
& E_{2, \chi_{1}, \chi_{5}}(z)=\sum_{n=1}^{\infty} \sigma_{\left(1, \chi_{1}, \chi_{5}\right)}(n) q^{n} .
\end{aligned}
$$


We recall that an eta quotient is of the following form

$$
\prod_{1 \leq \delta \mid N} \eta^{r} \delta(\delta z)
$$

where $N \in \mathbb{N}$ and $r_{\delta} \in \mathbb{Z}$, not all zero, for all $1 \leq \delta \mid N$. Note that, using Theorems 4.2.4 and 4.2.6, one can verify that eta quotients given in Tables 11.1.1 11.1.4 are complete lists of eta quotients in $M_{2}\left(\Gamma_{0}(8), \chi_{1}\right)$, $M_{2}\left(\Gamma_{0}(8), \chi_{8}\right), M_{2}\left(\Gamma_{0}(9), \chi_{1}\right), M_{2}\left(\Gamma_{0}(10), \chi_{5}\right)$, respectively.

By 11.1.1-11.1.4), we have the following results.

Theorem 11.1.1. Let $f(z) \in M_{2}\left(\Gamma_{0}(8), \chi_{1}\right)$ be an eta quotient given by

$$
f(z)=\prod_{1 \leq \delta \mid 8} \eta^{r_{\delta}}(\delta z) .
$$

Then

$$
f(z)=b_{1} E_{2}(z)+b_{2} E_{2}(2 z)+b_{3} E_{2}(4 z)+b_{4} E_{2}(8 z)
$$

for some $b_{1}, b_{2}, b_{3}, b_{4} \in \mathbb{Q}$, and the Fourier coefficients $a_{n}$ of the Fourier series $f(z)=\sum_{n=0}^{\infty} a_{n} q^{n}$ are given by

$$
\begin{aligned}
& a_{0}=-\left(b_{1}+b_{2}+b_{3}+b_{4}\right) / 24, \\
& a_{n}=b_{1} \sigma(n)+b_{2} \sigma(n / 2)+b_{3} \sigma(n / 4)+b_{4} \sigma(n / 8) \text { for } n \geq 1,
\end{aligned}
$$

where all eta quotients and corresponding values $b_{1}, b_{2}, b_{3}, b_{4}$ are given in Ta- 
ble 11.1.1.

Table 11.1.1: Values of $r_{1}, r_{2}, r_{4}, r_{8}, b_{1}, b_{2}, b_{3}, b_{4}$ for which

$$
\begin{aligned}
\eta^{r_{1}}(z) \eta^{r_{2}}(2 z) & \eta^{r_{4}}(4 z) \eta^{r_{8}}(8 z)=-\left(b_{1}+b_{2}+b_{3}+b_{4}\right) / 24 \\
+ & \sum_{n=1}^{\infty}\left(b_{1} \sigma(n)+b_{2} \sigma(n / 2)+b_{3} \sigma(n / 4)\right. \\
& \left.+b_{4} \sigma(n / 8)\right) q^{n}
\end{aligned}
$$

\begin{tabular}{rrrr|rrrr}
\hline \hline$r_{1}$ & $r_{2}$ & $r_{4}$ & $r_{8}$ & $b_{1}$ & $b_{2}$ & $b_{3}$ & $b_{4}$ \\
\hline-8 & 20 & -8 & 0 & 8 & 0 & -32 & 0 \\
-4 & 6 & 6 & -4 & 4 & -4 & 8 & -32 \\
-4 & 10 & -6 & 4 & 1 & 1 & -10 & 8 \\
0 & -8 & 20 & -8 & 0 & 8 & 0 & -32 \\
0 & -4 & 8 & 0 & 1 & -3 & 2 & 0 \\
0 & 0 & -4 & 8 & 0 & 1 & -3 & 2 \\
0 & 8 & -4 & 0 & 0 & -8 & 48 & -64 \\
4 & -6 & 10 & -4 & -4 & 20 & -8 & -32 \\
4 & -2 & -2 & 4 & 1 & -7 & 14 & -8 \\
8 & -4 & 0 & 0 & -8 & 48 & -64 & 0 \\
\hline
\end{tabular}

Theorem 11.1.2. Let $f(z) \in M_{2}\left(\Gamma_{0}(8), \chi_{8}\right)$ be an eta quotient given by

$$
f(z)=\prod_{1 \leq \delta \mid 8} \eta^{r_{\delta}}(\delta z)
$$


Then

$$
f(z)=b_{1} E_{2, \chi_{8}, \chi_{1}}(z)+b_{2} E_{2, \chi_{1}, \chi_{8}}(z)
$$

for some $b_{1}, b_{2} \in \mathbb{Q}$, and the coefficients $a_{n}$ of the Fourier series $f(z)=$ $\sum_{n=0}^{\infty} a_{n} e^{2 \pi i n z}$ are given by

$$
\begin{aligned}
& a_{0}=-b_{2} / 2, \\
& a_{n}=b_{1} \sigma_{\left(1, \chi_{1}, \chi_{8}\right)}(n)+b_{2} \sigma_{\left(1, \chi_{8}, \chi_{1}\right)}(n) \text { for } n \geq 1,
\end{aligned}
$$

where all eta quotients and corresponding values $b_{1}, b_{2}$ are given in Table 11.1 .2 .

Table 11.1.2: Values of $r_{1}, r_{2}, r_{4}, r_{8}, b_{1}, b_{2}$ for which

$$
\begin{aligned}
& \eta^{r_{1}}(z) \eta^{r_{2}}(2 z) \eta^{r_{4}}(4 z) \eta^{r_{8}}(8 z)= \\
& +\quad b_{2} / 2 \\
& +\sum_{n=1}^{\infty}\left(b_{1} \sigma_{\left(1, \chi_{1}, \chi_{8}\right)}(n) \quad+\right. \\
& \left.b_{2} \sigma_{\left(1, \chi_{8}, \chi_{1}\right)}(n)\right) q^{n}
\end{aligned}
$$

\begin{tabular}{rrrr|rr}
\hline \hline$r_{1}$ & $r_{2}$ & $r_{4}$ & $r_{8}$ & $b_{1}$ & $b_{2}$ \\
\hline-6 & 13 & -1 & -2 & 8 & -2 \\
-2 & -1 & 13 & -6 & 4 & -2 \\
-2 & 3 & 1 & 2 & 1 & 0 \\
2 & 1 & 3 & -2 & 0 & -2 \\
\hline
\end{tabular}


Theorem 11.1.3. Let $f(z) \in M_{2}\left(\Gamma_{0}(9), \chi_{1}\right)$ be an eta quotient given by

$$
f(z)=\prod_{1 \leq \delta \mid 9} \eta^{r_{\delta}}(\delta z)
$$

Then

$$
f(z)=b_{1} E_{2}(z)+b_{2} E_{2}(3 z)+b_{3} E_{2}(9 z)+b_{4} E_{2, \chi_{-3}, \chi_{-3}}(z)
$$

for some $b_{1}, b_{2}, b_{3}, b_{4} \in \mathbb{Q}$, and the Fourier coefficients $a_{n}$ of the Fourier series $f(z)=\sum_{n=0}^{\infty} a_{n} q^{n}$ are given by

$$
\begin{aligned}
& a_{0}=-\left(b_{1}+b_{2}+b_{3}\right) / 24 \\
& a_{n}=b_{1} \sigma(n)+b_{2} \sigma(n / 3)+b_{3} \sigma(n / 9)+b_{4} \sigma_{\left(1, \chi_{-3}, \chi_{-3}\right)}(n) \text { for } n \geq 1,
\end{aligned}
$$

where all eta quotients and corresponding values $b_{1}, b_{2}, b_{3}, b_{4}$ are given in Table 11.1.3. 
Table 11.1.3: Values of $r_{1}, r_{3}, r_{9}, b_{1}, b_{2}, b_{3}, b_{4}$ for which

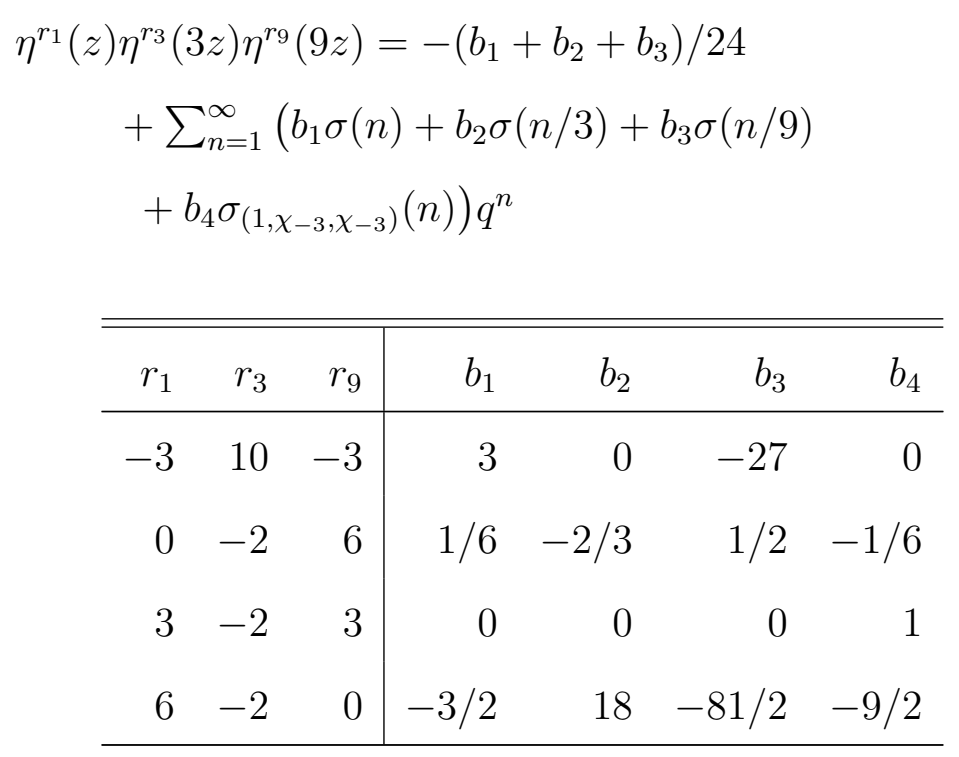

Theorem 11.1.4. Let $f(z) \in M_{2}\left(\Gamma_{0}(10), \chi_{5}\right)$ be an eta quotient given by

$$
f(z)=\prod_{1 \leq \delta \mid 10} \eta^{r_{\delta}}(\delta z)
$$

Then

$$
f(z)=b_{1} E_{2, \chi_{5}, \chi_{1}}(z)+b_{2} E_{2, \chi_{1}, \chi_{5}}(z)+b_{3} E_{2, \chi_{5}, \chi_{1}}(2 z)+b_{4} E_{2, \chi_{1}, \chi_{5}}(2 z)
$$

for some $b_{1}, b_{2}, b_{3}, b_{4} \in \mathbb{Q}$, and the Fourier coefficients $a_{n}$ of the Fourier series 


$$
\begin{aligned}
f(z) & =\sum_{n=0}^{\infty} a_{n} q^{n} \text { are given by } \\
a_{0} & =-\left(b_{1}+b_{3}\right) / 5, \\
a_{n} & =b_{1} \sigma_{\left(1, \chi_{5}, \chi_{1}\right)}(n)+b_{2} \sigma_{\left(1, \chi_{1}, \chi_{5}\right)}(n)+b_{3} \sigma_{\left(1, \chi_{5}, \chi_{1}\right)}(n / 2)+b_{4} \sigma_{\left(1, \chi_{1}, \chi_{5}\right)}(n / 2) \text { for } n \geq 1,
\end{aligned}
$$

where all eta quotients and corresponding values $b_{1}, b_{2}, b_{3}, b_{4}$ are given in Table 11.1.4.

Table 11.1.4: Values of $r_{1}, r_{2}, r_{5}, r_{10}, b_{1}, b_{2}, b_{3}$ for which

$$
\begin{aligned}
& \eta^{r_{1}}(z) \eta^{r_{2}}(2 z) \eta^{r_{5}}(5 z) \eta^{r_{10}}(10 z)=-\left(b_{1}+b_{3}\right) / 5 \\
& \quad+\sum_{n=1}^{\infty}\left(b_{1} \sigma_{\left(1, \chi_{5}, \chi_{1}\right)}(n)+b_{2} \sigma_{\left(1, \chi_{1}, \chi_{5}\right)}(n)+\right. \\
& b_{3} \sigma_{\left(1, \chi_{5}, \chi_{1}\right)}(n / 2) \\
& \left.\quad+b_{4} \sigma_{\left(1, \chi_{1}, \chi_{5}\right)}(n / 2)\right) q^{n}
\end{aligned}
$$

\begin{tabular}{rrrr|rrrr}
\hline \hline$r_{1}$ & $r_{2}$ & $r_{5}$ & $r_{10}$ & $b_{1}$ & $b_{2}$ & $b_{3}$ & $b_{4}$ \\
\hline-5 & 10 & 1 & -2 & $-5 / 4$ & $25 / 4$ & $-15 / 4$ & $25 / 4$ \\
-4 & 7 & 4 & -3 & -1 & 5 & -4 & 5 \\
-3 & 4 & 7 & -4 & -1 & 4 & -4 & 4 \\
-3 & 6 & -1 & 2 & $-1 / 4$ & $5 / 4$ & $1 / 4$ & $5 / 4$ \\
-2 & 1 & 10 & -5 & -1 & 3 & -4 & 4 \\
-2 & 3 & 2 & 1 & 0 & 1 & 0 & 1 \\
-1 & 0 & 5 & 0 & 0 & 1 & 0 & 0 \\
-1 & 2 & -3 & 6 & $-1 / 4$ & $1 / 4$ & $1 / 4$ & $1 / 4$ \\
\hline
\end{tabular}




\begin{tabular}{rrrr|rrrr}
\hline \hline$r_{1}$ & $r_{2}$ & $r_{5}$ & $r_{10}$ & $b_{1}$ & $b_{2}$ & $b_{3}$ & $b_{4}$ \\
\hline 0 & -1 & 0 & 5 & 0 & 0 & 0 & 1 \\
0 & 5 & 0 & -1 & 0 & 0 & -5 & 0 \\
1 & 2 & 3 & -2 & -1 & 0 & -4 & 0 \\
2 & -1 & 6 & -3 & -1 & -1 & -4 & 4 \\
2 & 1 & -2 & 3 & 1 & 0 & -1 & 0 \\
3 & -2 & 1 & 2 & 0 & 1 & 0 & -4 \\
5 & 0 & -1 & 0 & -5 & 0 & 0 & 0 \\
6 & -3 & 2 & -1 & -1 & -5 & -4 & 20 \\
\hline
\end{tabular}

Proofs of Theorems 11.1.1 11.1.4. We only prove Theorem 11.1.1 as the others can be proven similarly. Let us consider the eta quotient $\frac{\eta^{20}(4 z)}{\eta^{8}(2 z) \eta^{8}(8 z)} \in$ $M_{2}\left(\Gamma_{0}(8), \chi_{1}\right)$. By (1.0.1), Theorem 3.2.2 and (11.1.1) we have

$$
\frac{\eta^{20}(4 z)}{\eta^{8}(2 z) \eta^{8}(8 z)}=b_{1} E_{2}(z)+b_{2} E_{2}(2 z)+b_{3} E_{2}(4 z)+b_{4} E_{2}(8 z)
$$

for some $b_{1}, b_{2}, b_{3}, b_{4} \in \mathbb{Q}$. On the other hand, using MAPLE we compute the first 5 coefficients of the eta quotient.

$$
\frac{\eta^{20}(4 z)}{\eta^{8}(2 z) \eta^{8}(8 z)}=1+8 q^{2}+24 q^{4}+O\left(q^{5}\right) .
$$

Comparing the coefficients of $q^{0}, q^{1}, q^{2}, q^{3}, q^{4}$ of the right hand sides of 11.1.5 
and 11.1.6 we have

$-\left(b_{1}+b_{2}+b_{3}+b_{4}\right) / 24=1, b_{1}=0,3 b_{1}+b_{2}=8,4 b_{1}=0, b_{3}+3 b_{2}+7 b_{1}=24$

from which we obtain

$$
b_{1}=0, b_{2}=8, b_{3}=0, b_{4}=-32 .
$$

Similarly we determine the coefficients $b_{1}, b_{2}, b_{3}, b_{4}$ for the remaining eta quotients.

Note that the eta quotients in rows 7 and 15 in Table 11.1.4 first appear in Ramanujan's lost notebook, see [11, 64]. Using similar arguments it is possible to give Fourier coefficients for many eta quotients, see for example [5].

\subsection{A Proof for Ramanujan's Congruence for $p(n)$ Modulo 5}

The partition function, denoted by $p(n)$, is the number of different ways we can write $n$ as sums of positive integers. As an application of one of our results from Table 11.1.4, we present a proof for Ramanujan's congruence for the partition function $p(n)$ modulo 5, see [17, Theorem 4.4.1], 63].

Theorem 11.2.1. $p(5 n+4) \equiv 0(\bmod 5)$ for all $n \in \mathbb{N}_{0}$. 
Proof. We consider the eta quotient

$$
\frac{\eta^{5}(5 z)}{\eta(z)}
$$

from Table 11.1.4. We note that

$$
\frac{\eta^{5}(5 z)}{\eta(z)}=\prod_{n \geq 1}\left(1-q^{5 n}\right) \sum_{n=0}^{\infty} p(n) q^{n+1}
$$

Appealing to 11.2.1, 11.2.2 and Table 11.1.4 we have

$$
\sum_{n=1}^{\infty}\left(\sum_{d \mid n}\left(\frac{5}{n / d}\right) d\right) q^{n}=\frac{\eta^{5}(5 z)}{\eta(z)}=\prod_{n \geq 1}\left(1-q^{5 n}\right)^{5} \sum_{n=0}^{\infty} p(n) q^{n+1}
$$

We have

$$
\prod_{n \geq 1}\left(1-q^{5 n}\right)^{5}=1+\sum_{n \geq 1} a_{n} q^{5 n},\left(\text { with some } a_{n} \in \mathbb{Z}\right)
$$

Thus $p(5 n+4) \equiv 0(\bmod 5)$, for all $n \in \mathbb{N}_{0}$, if and only if the coefficient of $q^{5 n+5}$ on the left hand side of 11.2 .3 is divisible by 5 for all $n \in \mathbb{N}_{0}$. So to complete the proof we need to show that

$$
\sum_{d \mid 5 n+5}\left(\frac{5}{(5 n+5) / d}\right) d \equiv 0 \quad(\bmod 5)
$$

Let $d \mid 5 n+5$. If $5 \nmid d$ then $\left(\frac{5}{(5 n+5) / d}\right)=0$, and if $5 \mid d$ then 
$\left(\frac{5}{(5 n+5) / d}\right) d \equiv 0(\bmod 5)$, from which 11.2 .4 follows.

\subsection{Arithmetic Properties of Fourier Coef- ficients of Eta Quotients in $M_{2}\left(\Gamma_{0}(10), \chi_{5}\right)$}

We now use results of Theorem 11.1.4 to present some conditions for vanishing of certain Fourier coefficients of eta quotients in $M_{2}\left(\Gamma_{0}(10), \chi_{5}\right)$, together with some congruence relations for the Fourier coefficients of eta quotients in the same space.

Theorem 11.3.1. Let $f(z) \in M_{2}\left(\Gamma_{0}(10), \chi_{5}\right)$ be an eta quotient as in Theorem 11.1.4, that is $f(z)=\eta^{r_{1}}(z) \eta^{r_{2}}(2 z) \eta^{r_{5}}(5 z) \eta^{r_{10}}(10 z)$ with the Fourier series

$$
\begin{aligned}
f(z)= & \frac{-\left(b_{1}+b_{3}\right)}{5} \\
& \quad+\sum_{n=1}^{\infty}\left(b_{1} \sigma_{\left(1, \chi_{5}, \chi_{1}\right)}(n)+b_{2} \sigma_{\left(1, \chi_{1}, \chi_{5}\right)}(n)+b_{3} \sigma_{\left(1, \chi_{5}, \chi_{1}\right)}(n / 2)+b_{4} \sigma_{\left(1, \chi_{1}, \chi_{5}\right)}(n / 2)\right) q^{n},
\end{aligned}
$$

for some $b_{1}, b_{2}, b_{3}, b_{4} \in \mathbb{Q}$. Then for all $n \in \mathbb{N}_{0}$ we have

$$
\begin{aligned}
& a_{10 n+1}=a_{10 n+9}=0 \quad \text { if } b_{1}+b_{2}=0, \\
& a_{10 n+3}=a_{10 n+7}=0 \quad \text { if } b_{1}-b_{2}=0, \\
& a_{5 n+1}=a_{5 n+4}=0 \quad \text { if } b_{1}+b_{2}=b_{3}-b_{4}=0, \\
& a_{5 n+2}=a_{5 n+3}=0 \quad \text { if } b_{1}-b_{2}=b_{3}+b_{4}=0 ;
\end{aligned}
$$


and

$$
\begin{aligned}
& a_{10 n+1} \equiv a_{10 n+9} \equiv 0 \quad\left(\bmod \left|b_{1}+b_{2}\right|\right) \\
& a_{10 n+3} \equiv a_{10 n+7} \equiv 0 \quad\left(\bmod \left|b_{1}-b_{2}\right|\right) \\
& a_{5 n+1} \equiv a_{5 n+4} \equiv 0 \quad(\bmod s) \quad \text { if } \operatorname{gcd}\left(b_{1}+b_{2}, b_{3}-b_{4}\right)=s \\
& a_{5 n+2} \equiv a_{5 n+3} \equiv 0 \quad(\bmod t) \quad \text { if } \operatorname{gcd}\left(b_{1}-b_{2}, b_{3}+b_{4}\right)=t
\end{aligned}
$$

Proof. Let $5 \nmid n$. For all $d \mid n$ we have

$$
\left(\frac{5}{n / d}\right)\left(\frac{5}{d}\right)=\left(\frac{5}{n}\right)
$$

We also have

$$
\left(\frac{5}{n}\right)=\left\{\begin{aligned}
1 & \text { if } n \equiv 1,4 \quad(\bmod 5) \\
-1 \quad \text { if } n & \equiv 2,3 \quad(\bmod 5)
\end{aligned}\right.
$$

It follows from 11.3.1 and 11.3.2 that

$$
\left(\frac{5}{n / d}\right)=\left\{\begin{aligned}
\left(\frac{5}{d}\right) & \text { if } n \equiv 1,4 \quad(\bmod 5) \\
-\left(\frac{5}{d}\right) & \text { if } n \equiv 2,3 \quad(\bmod 5)
\end{aligned}\right.
$$

Hence we have

$$
\sigma_{\left(1, \chi_{1}, \chi_{5}\right)}(n)=\left\{\begin{aligned}
\sigma_{\left(1, \chi_{5}, \chi_{1}\right)}(n) & \text { if } n \equiv 1,4 \quad(\bmod 5) \\
-\sigma_{\left(1, \chi_{5}, \chi_{1}\right)}(n) & \text { if } n \equiv 2,3 \quad(\bmod 5)
\end{aligned}\right.
$$


The results now follow from 11.3.3 and Theorem 11.1.4.

\subsection{Number of Eta Quotients in $E_{2}\left(\Gamma_{0}(N)\right)$ for $N<100$}

In spaces of modular forms which have cusp form space of nonzero dimension we can still produce similar results for some of the eta quotients using the techniques given in this chapter. However it seems difficult to give a complete set of results as we did in first section of this chapter. In Figure 11.4.1 below we give the number of eta quotients in $E_{2}\left(\Gamma_{0}(N)\right)$. The abundance and similarities of such eta quotients suggest that there might be a set of criteria to determine if a given eta quotient is in $E_{2}\left(\Gamma_{0}(N)\right)$. 


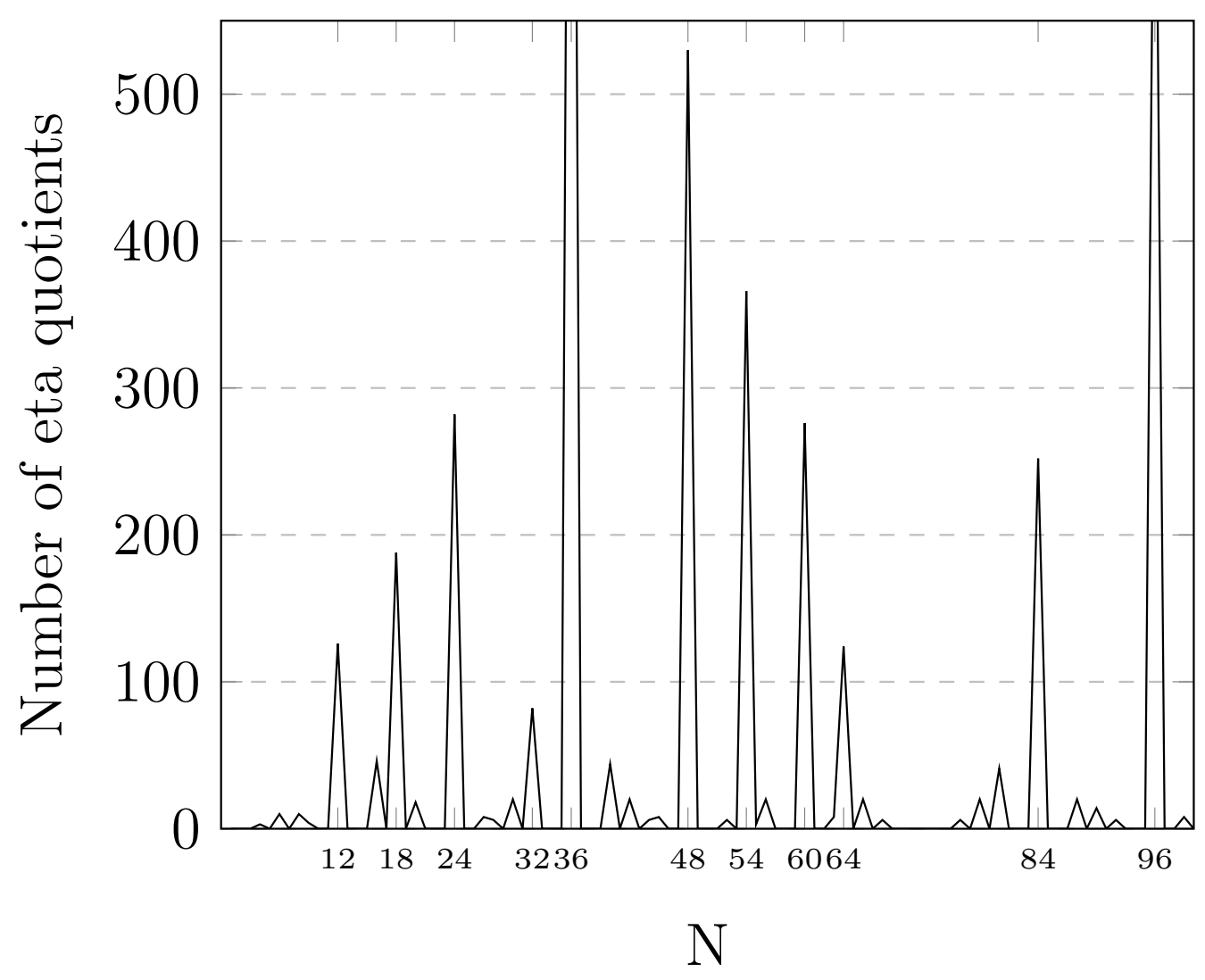

Figure 11.4.1: Number of eta quotients in $E_{2}\left(\Gamma_{0}(N)\right)$ 


\section{Chapter 12}

\section{Future Research and Conclusion}

In this chapter we talk about other possible directions that we can stretch results of this thesis. We will also try to describe other possible applications of some of the results of this thesis, along with a description of what we want to achieve in the future.

\subsection{Bases for $S_{k}\left(\Gamma_{0}(N), \chi\right)$ in Terms of Eta Quotients}

In Chapter 5, we gave bases for $S_{k}\left(\Gamma_{0}(12), \chi\right)$ for all nontrivial spaces with $k \in \mathbb{N}$ and $\chi$ a real character in terms of eta quotients. In the Appendix A, we give bases for $S_{k}\left(\Gamma_{0}(N)\right)$ for $N=20$ and 28. All these eta quotients have different orders at infinity. Among many others $S_{2 k}\left(\Gamma_{0}(24)\right)$ is a good candidate to have such a basis. We can produce a basis for $S_{2 k}\left(\Gamma_{0}(24)\right)$ for 
all $k>1$ in terms of eta quotients whose orders at infinity are different. We can achieve this by analyzing the orders of cusps of the eta quotients in $S_{2 k}\left(\Gamma_{0}(24)\right)$, for $k=2,3, \ldots, k^{\prime}$ where $k^{\prime}$ is a number to be determined.

Let $k>1$ be an integer. It would be interesting to find a set of criteria on divisors of $N$ for which $S_{2 k}\left(\Gamma_{0}(N)\right)$ has a basis in terms of eta quotients whose orders at infinity are different. We close this section with stating our prediction that if divisors of $N$ are not 'sparsely' distributed then for some $k^{\prime} \in \mathbb{N}$, we can find a basis for $S_{2 k}\left(\Gamma_{0}(N)\right)$ for all $k>k^{\prime}$ in terms of eta quotients whose orders at infinity are different.

\subsection{Extensions of Ramanujan-Mordell For- mula}

In Chapter 7, we gave a formula for $N\left(1^{4 k-2 i}, 3^{2 i} ; n\right)$, for all $k>2$ and $0 \leq i \leq 2 k$. It is possible to give more of these extensions. Since the bases for $S_{2 k}\left(\Gamma_{0}(20)\right)$ and $S_{2 k}\left(\Gamma_{0}(28)\right)$ for all $k \geq 1$ are given in Appendix $\mathrm{A}$, an immediate extension would be finding the formulas for $N\left(1^{4 k-2 i}, 5^{2 i} ; n\right)$ and $N\left(1^{4 k-2 i}, 7^{2 i} ; n\right)$. We expect that the formulas would be pretty similar to the one given by Theorem 7.1.3, except this time we will need to use the bases for $S_{2 k}\left(\Gamma_{0}(20)\right)$ and $S_{2 k}\left(\Gamma_{0}(28)\right)$ given in Appendix A. For the proof one should compute a table similar to Table 7.1.1 by using Theorem 6.2.1 and carrying out computations similar to the ones from Section 6.1. Then one should find a system of linear equations similar to the one given after Table 7.1.1, 
solving this system will give the desired formula. Interested readers should be careful about giving the coefficients of cusp forms. This process is not limited to $N=12,20$, or 28 . Let $N_{1} \in \mathbb{N}$, once a basis for $S_{2 k}\left(\Gamma_{0}\left(4 \cdot N_{1}\right)\right)$ is given, we can derive formulas for

$$
\prod_{1 \geq d \mid N_{1}} \varphi^{l_{d}}(d z)
$$

where $\sum_{1 \leq d \mid N_{1}} l_{d}=4 k$ with $\sum_{d / p \in \mathbb{N}} l_{d}$ an even number for all prime $p \mid N_{1}$. A formula for 12.2.1 will yield to a formula for $N\left(1^{l_{1}}, \ldots, d^{l_{d}}, \ldots, N_{1}^{l_{N_{1}}}, n\right)$.

We end this section by stating our predictions for formulas for $N\left(1^{2 k-i}, 3^{i} ; n\right)$ for $k \in \mathbb{N}$ and $0 \leq i \leq 2 k$, except the cases given by Theorem 7.1.3.

Conjecture 12.2.1. Let $i, j, k \in \mathbb{N}$ such that $k>1,0 \leq i \leq 2 k-1$ and $1 \leq j \leq 4 k-4$. Then we have

$$
\begin{aligned}
\varphi^{2 i+1}(z) \varphi^{4 k-2 i-1}(3 z)= & b_{(1, i)} \sum_{n=1}^{\infty} \sigma_{\left(2 k-1, \chi_{-4}, \chi_{-3}\right)}(n) q^{n}+b_{(2, i)} \sum_{n=1}^{\infty} \sigma_{\left(2 k-1, \chi_{12}, \chi_{1}\right)}(n) q^{n} \\
& +b_{(3, i)} \sum_{n=1}^{\infty} \sigma_{\left(2 k-1, \chi_{1}, \chi_{12}\right)}(n) q^{n}+b_{(4, i)} \sum_{n=1}^{\infty} \sigma_{\left(2 k-1, \chi_{-3}, \chi_{-4}\right)}(n) q^{n} \\
& +\sum_{1 \leq j \leq 4 k-4} c_{j} D_{j, 2 k}(z)
\end{aligned}
$$

where

$$
b_{(1, i)}=\frac{(-1)^{i+1} 2^{2 k-1}}{B_{2 k, \chi_{12}}}, b_{(2, i)}=\frac{(-1)^{k+i+1} 3^{i}}{B_{2 k, \chi_{12}}}, b_{(3, i)}=\frac{(-1)^{k}}{B_{2 k, \chi_{12}}}, b_{(4, i)}=\frac{(-1)^{i+1} 2^{2 k-1} 3^{i}}{B_{2 k, \chi_{12}}},
$$


and

$$
\begin{aligned}
c_{j}= & {[j] \varphi^{2 i+1}(z) \varphi^{4 k-2 i-1}(3 z) } \\
& -\left(b_{(1, i)} \sigma_{\left(2 k-1, \chi_{-4}, \chi_{-3}\right)}(j)+b_{(2, i)} \sigma_{\left(2 k-1, \chi_{12}, \chi_{1}\right)}(j)\right. \\
& +b_{(3, i)} \sigma_{\left(2 k-1, \chi_{1}, \chi_{12}\right)}(j)+b_{(4, i)} \sigma_{\left(2 k-1, \chi_{-3}, \chi_{-4}\right)}(j) \\
& \left.+[j] \sum_{1 \leq l \leq j-1} c_{l} D_{l, 2 k}(z)\right)
\end{aligned}
$$

Conjecture 12.2.2. Let $i, j, k \in \mathbb{N}$ such that $k>1,0 \leq i \leq 2 k-1$ and $1 \leq j \leq 4 k-3$. Then we have

$$
\begin{aligned}
& \varphi^{2 i+1}(z) \varphi^{4 k-2 i+1}(3 z)= \\
& b_{(1, i)} \sum_{n=1}^{\infty} \sigma_{\left(2 k, \chi_{-3}, \chi_{1}\right)}(n) q^{n}+b_{(2, i)} \sum_{n=1}^{\infty} \sigma_{\left(2 k, \chi_{-3}, \chi_{1}\right)}(n) q^{2 n}+b_{(3, i)} \sum_{n=1}^{\infty} \sigma_{\left(2 k, \chi_{-3}, \chi_{1}\right)}(n) q^{4 n} \\
& +b_{(4, i)} \sum_{n=1}^{\infty} \sigma_{\left(2 k, \chi_{1}, \chi_{-3}\right)}(n) q^{n}+b_{(6, i)} \sum_{n=1}^{\infty} \sigma_{\left(2 k, \chi_{1}, \chi_{-3}\right)}(n) q^{2 n}+b_{(12, i)} \sum_{n=1}^{\infty} \sigma_{\left(2 k, \chi_{1}, \chi_{-3}\right)}(n) q^{4 n} \\
& +\sum_{1 \leq j \leq 4 k-3} c_{j} C_{j, 2 k+1}(z)
\end{aligned}
$$

where

$$
\begin{aligned}
& b_{(1, i)}=-\frac{(-1)^{i}}{\left(2^{2 k+1}+1\right) B_{2 k+1, \chi_{-3}}} \\
& b_{(2, i)}=\frac{(-1)^{k}\left(1+(-1)^{k+i}\right)}{\left(2^{2 k+1}+1\right) B_{2 k+1, \chi_{-3}}} \\
& b_{(3, i)}=\frac{(-1)^{k} 2^{2 k+1}}{\left(2^{2 k+1}+1\right) B_{2 k+1, \chi_{-3}}}, \\
& b_{(4, i)}=\frac{3^{i}}{\left(2^{2 k+1}+1\right) B_{2 k+1, \chi_{-3}}},
\end{aligned}
$$




$$
\begin{aligned}
& b_{(6, i)}=\frac{\left(1+(-1)^{k+i}\right) 3^{i}}{\left(2^{2 k+1}+1\right) B_{2 k+1, \chi_{-3}}} \\
& b_{(12, i)}=\frac{(-1)^{i+k} 2^{2 k+1} 3^{i}}{\left(2^{2 k+1}+1\right) B_{2 k+1, \chi_{-3}}}
\end{aligned}
$$

and

$$
\begin{aligned}
c_{j}= & {[j] \varphi^{2 i+1}(z) \varphi^{4 k-2 i+1}(3 z) } \\
& \left(b_{(1, i)} \sigma_{\left(2 k, \chi_{-3}, \chi_{1}\right)}(j)+b_{(2, i)} \sigma_{\left(2 k, \chi_{-3}, \chi_{1}\right)}(j / 2)+b_{(3, i)} \sigma_{\left(2 k, \chi_{-3}, \chi_{1}\right)}(j / 4)\right. \\
& +b_{(4, i)} \sigma_{\left(2 k, \chi_{1}, \chi_{-3}\right)}(j)+b_{(6, i)} \sigma_{\left(2 k, \chi_{1}, \chi_{-3}\right)}(j / 2)+b_{(12, i)} \sigma_{\left(2 k, \chi_{1}, \chi_{-3}\right)}(j / 4) \\
& \left.+[j] \sum_{1 \leq l \leq j-1} c_{l} C_{l, 2 k+1}(z)\right)
\end{aligned}
$$

Conjecture 12.2.3. Let $i, j, k \in \mathbb{N}$ such that $k>1,0 \leq i \leq 2 k-1$ and $1 \leq j \leq 4 k-2$. Then we have

$$
\begin{aligned}
\varphi^{2 i}(z) \varphi^{4 k-2 i+2}(3 z)= & b_{(1, i)} \sum_{n=1}^{\infty} \sigma_{\left(2 k, \chi_{-4}, \chi_{1}\right)}(n) q^{n}+b_{(2, i)} \sum_{n=1}^{\infty} \sigma_{\left(2 k, \chi_{-4}, \chi_{1}\right)}(n) q^{3 n} \\
& +b_{(3, i)} \sum_{n=1}^{\infty} \sigma_{\left(2 k, \chi_{1}, \chi_{-4}\right)}(n) q^{n}+b_{(4, i)} \sum_{n=1}^{\infty} \sigma_{\left(2 k, \chi_{1}, \chi_{-4}\right)}(n) q^{3 n} \\
& +\sum_{1 \leq j \leq 4 k-2} c_{j} D_{j, 2 k+1}(z)
\end{aligned}
$$

where

$$
\begin{aligned}
& b_{(1, i)}=-\frac{(-1)^{i}\left(3^{i}+(-1)^{i+1}\right)}{3^{2 k+1} B_{2 k+1, \chi-4}}, \\
& b_{(2, i)}=\frac{(-1)^{k} 3^{i}\left(3^{2 k+1-i}+(-1)^{2 k-i}\right)}{3^{2 k+1} B_{2 k+1, \chi-4}},
\end{aligned}
$$




$$
\begin{aligned}
& b_{(3, i)}=-\frac{-\left(3^{i}+(-1)^{i+1}\right)\left(2^{2 k}\right)}{3^{2 k+1} B_{2 k+1, \chi_{-4}}}, \\
& b_{(4, i)}=\frac{-(-1)^{i} 3^{i}\left(3^{2 k+1-i}+(-1)^{2 k-i}\right)\left(2^{2 k}\right)}{3^{2 k+1} B_{2 k+1, \chi-4}},
\end{aligned}
$$

and

$$
\begin{aligned}
c_{j}= & {[j] \varphi^{2 i}(z) \varphi^{4 k-2 i+2}(3 z) } \\
& \left(b_{(1, i)} \sigma_{\left(2 k, \chi_{-4}, \chi_{1}\right)}(j)+b_{(2, i)} \sigma_{\left(2 k, \chi_{-4}, \chi_{1}\right)}(j / 3)\right. \\
& +b_{(3, i)} \sigma_{\left(2 k, \chi_{1}, \chi_{-4}\right)}(j)+b_{(4, i)} \sigma_{\left(2 k, \chi_{1}, \chi_{-4}\right)}(j / 3) \\
& \left.+[j] \sum_{1 \leq l \leq j-1} c_{l} D_{l, 2 k+1}(z)\right)
\end{aligned}
$$

Proof of these statements require finding first terms of Fourier series expansions of $E_{k, \chi, \psi}(z)$ at each cusp, i.e. an analogue of Theorem 6.1.1. In [22] a similar calculation was done at the cusp 0 .

\subsection{Half-Integral Weight Modular Forms}

Earlier we noted that in Definition 2.3.1 we restrict the weight to be an integer and the multiplier system to be a real character. We can relax these conditions. We now let weight be in $\mathbb{N}+\frac{1}{2}$ and multiplier system $\chi$ be a complex function with norm 1 . Then a function which satisfies (i) and (ii) of Definition 2.3.1 is called a modular form with half integral weight. For more information and some technical details see [40, Section 1.3, p. 14] and [39, p. 182]. 
Let $k^{\prime} \in \mathbb{N}$ and $1 \leq j \leq 4 k^{\prime}-3$. Consider the following weight $2 k^{\prime}+1 / 2$ eta quotients

$$
\begin{aligned}
& F_{2 k^{\prime}+1 / 2, j}(z)=\frac{\eta^{3 j-3}(z) \eta^{2 k^{\prime}-2 j+3}(2 z) \eta^{12 k^{\prime}-3 j-7}(12 z)}{\eta^{j-3}(3 z) \eta^{4 k^{\prime}-j-3}(4 z) \eta^{6 k^{\prime}-2 j-2}(6 z)}, \\
& G_{2 k^{\prime}+1 / 2, j}(z)=\frac{\eta^{3 j-1}(z) \eta^{2 k^{\prime}-2 j-2}(2 z) \eta^{12 k^{\prime}-3 j-9}(12 z)}{\eta^{j-1}(3 z) \eta^{4 k^{\prime}-j-5}(4 z) \eta^{6 k^{\prime}-2 j-7}(6 z)}, \\
& \varphi^{4 k^{\prime}-2 i+1}(z) \varphi^{2 i}(3 z)=\frac{\eta^{20 k^{\prime}-10 i+5}(2 z) \eta^{10 i}(6 z)}{\eta^{8 k^{\prime}-4 i+2}(z) \eta^{4 i}(3 z) \eta^{8 k^{\prime}-4 i+2}(4 z) \eta^{4 i}(12 z)}, \\
& \varphi^{4 k^{\prime}-2 i}(z) \varphi^{2 i+1}(3 z)=\frac{\eta^{20 k^{\prime}-10 i}(2 z) \eta^{10 i+5}(6 z)}{\eta^{8 k^{\prime}-4 i}(z) \eta^{4 i+2}(3 z) \eta^{8 k^{\prime}-4 i}(4 z) \eta^{4 i+2}(12 z)}
\end{aligned}
$$

By (4.1.4), the eta quotients given by (12.3.1)-(12.3.4) are holomorphic at all cusps of $\Gamma_{0}(12)$, i.e. they satisfy Definition 2.3.1 (ii). By (4.1.3) the eta quotients given by 12.3.1) satisfy the same transformation formula for all $1 \leq j \leq 4 k^{\prime}-3$, and so they belong to the same modular form space, say $M_{2 k^{\prime}+1 / 2}\left(\Gamma_{0}(12), \chi\right)$. Again by (4.1.3) the eta quotients given by (12.3.2) satisfy the same transformation formula for all $1 \leq j \leq 4 k^{\prime}-3$, also proving that they belong to the same modular form space, say $M_{2 k^{\prime}+1 / 2}\left(\Gamma_{0}(12), \chi^{\prime}\right)$. Using dimension formulas for half integral weight modular forms in [23, $\mathrm{p}$. 75 ] and the fact that their orders at all cusps are always strictly greater than 0 one can show that the set of eta quotients $\left\{F_{2 k^{\prime}+1 / 2, j}(z)\right\}_{1 \leq j \leq 4 k^{\prime}-3}$ and $\left\{G_{2 k^{\prime}+1 / 2, j}(z)\right\}_{1 \leq j \leq 4 k^{\prime}-3}$ constitute a basis for $S_{2 k^{\prime}+1 / 2}\left(\Gamma_{0}(12), \chi\right)$ and $S_{2 k^{\prime}+1 / 2}\left(\Gamma_{0}(12), \chi^{\prime}\right)$, respectively. By 4.1.3 we also have

$$
\varphi^{4 k^{\prime}-2 i+1}(z) \varphi^{2 i}(3 z) \in M_{2 k^{\prime}+1 / 2}\left(\Gamma_{0}(12), \chi\right),
$$




$$
\varphi^{4 k^{\prime}-2 i}(z) \varphi^{2 i+1}(3 z) \in M_{2 k^{\prime}+1 / 2}\left(\Gamma_{0}(12), \chi^{\prime}\right) .
$$

We wish to find bases for $E_{2 k^{\prime}+1 / 2}\left(\Gamma_{0}(12), \chi\right)$ and $E_{2 k^{\prime}+1 / 2}\left(\Gamma_{0}(12), \chi^{\prime}\right)$ so that we can express $\varphi^{4 k^{\prime}-2 i+1}(z) \varphi^{2 i}(3 z)$ and $\varphi^{4 k^{\prime}-2 i}(z) \varphi^{2 i+1}(3 z)$ as linear combintaions of Eisenstein series and cusp forms as we have done in Chapter 7 .

Our approach would be as follows. Suppose the sets

$$
\begin{aligned}
& \left\{E_{\left(k^{\prime}, 1\right)}(z), E_{\left(k^{\prime}, 2\right)}(z), E_{\left(k^{\prime}, 3\right)}(z), E_{\left(k^{\prime}, 4\right)}(z)\right\}, \\
& \left\{E_{\left(k^{\prime}, 1\right)}^{\prime}(z), E_{\left(k^{\prime}, 2\right)}^{\prime}(z), E_{\left(k^{\prime}, 3\right)}^{\prime}(z), E_{\left(k^{\prime}, 4\right)}^{\prime}(z)\right\}
\end{aligned}
$$

constitute bases for $E_{2 k^{\prime}+1 / 2}\left(\Gamma_{0}(12), \chi\right)$ and $E_{2 k^{\prime}+1 / 2}\left(\Gamma_{0}(12), \chi^{\prime}\right)$, respectively. Then we would have

$$
\begin{aligned}
\varphi^{4 k^{\prime}-2 i+1}(z) \varphi^{2 i}(3 z)= & b_{1} E_{\left(k^{\prime}, 1\right)}(z)+b_{2} E_{\left(k^{\prime}, 1\right)}(z)+b_{3} E_{\left(k^{\prime}, 1\right)}(z)+b_{4} E_{\left(k^{\prime}, 1\right)}(z) \\
& +\sum_{1 \leq j \leq 4 k^{\prime}-3} c_{j} F_{2 k^{\prime}+1 / 2, j}(z), \\
\varphi^{4 k^{\prime}-2 i}(z) \varphi^{2 i+1}(3 z)= & b_{1}^{\prime} E_{\left(k^{\prime}, 1\right)}^{\prime}(z)+b_{2}^{\prime} E_{\left(k^{\prime}, 1\right)}^{\prime}(z)+b_{3}^{\prime} E_{\left(k^{\prime}, 1\right)}^{\prime}(z)+b_{4}^{\prime} E_{\left(k^{\prime}, 1\right)}^{\prime}(z) \\
& +\sum_{1 \leq j \leq 4 k^{\prime}-3} c_{j}^{\prime} G_{2 k^{\prime}+1 / 2, j}(z)
\end{aligned}
$$

for some coefficients $b_{i}, b_{i}^{\prime}, c_{j}, c_{j}^{\prime} \in \mathbb{C}$. We now need to determine the bases for $E_{2 k^{\prime}+1 / 2}\left(\Gamma_{0}(12), \chi\right)$ and $E_{2 k^{\prime}+1 / 2}\left(\Gamma_{0}(12), \chi^{\prime}\right)$. See [39, Section IV.2], [37], [73, Part 1, Section D] for Eisenstein series in half integral weight modular form spaces. Then we need to determine the coefficients in 12.3.5 and 12.3.6). If we fix $k^{\prime}$, we can find coefficients by comparing the first few Fourier co- 
efficients of both sides in $(12.3 .5)$ and $(12.3 .6)$. To give a general statement similar to Theorem 7.1.2 we need to give an analogue of Theorem 6.2.1 for half integral weight Eisenstein series. Then 12.3 .5 and 12.3 .6 would yield formulas for $N\left(1^{4 k^{\prime}-2 i+1}, 3^{2 i} ; n\right)$ and $N\left(1^{4 k^{\prime}-2 i}, 3^{2 i+1} ; n\right)$, respectively. See [33] for an application of half integral weight modular forms to representations with ternary quadratic forms.

\subsection{Application to Convolution Sums}

A formula for the convolution sum

$$
\sum_{a+b=n} \sigma(a) \sigma(b)
$$

is known, see [29]. A number of variations of this sum have been studied by A. Alaca, S. Alaca, S. Cooper and K. S. Williams, among others. Let $p$ denote a prime number. Consider the modular form $\left(L_{p}(z)\right)^{2}$ in $M_{4}\left(\Gamma_{0}(p)\right)$. Then by Corollary 3.3.1 and (1.0.1), we have

$$
\left(L_{p}(z)\right)^{2}=b_{1} E_{4}(z)+b_{2} E_{4}(p z)+C_{p}(z),
$$

where $L_{p}(z), E_{4}(z)$ and $E_{4}(p z)$ are as defined in Section 3.2 , and $C_{p}(z)$ is a cusp form in $S_{4}\left(\Gamma_{0}(p)\right)$. To complete the formula in 12.4 .2 there are two challenges. First we need to find $b_{1}$ and $b_{2}$, second we need to describe the cusp part $C_{p}(z)$. 
We can compute $b_{1}$ and $b_{2}$ by comparing the first terms of $L_{p}(z)$ in its Fourier series expansions at cusps 1 (not known) and $\infty$ (given in 3.2.3), to the first terms of the Fourier series expansions of $b_{1} E_{4}(z)$ and $b_{2} E_{4}(p z)$ at cusps 1 (given in 6.2.1) and $\infty$ (given in (6.2.1) ).

For small $p$ one can give $C_{p}(z)$ in terms of eta quotients, or alternatively see 42 for the bases for $S_{4}\left(\Gamma_{0}(p)\right)$. Then by comparing $n$th coefficients of both sides of 12.4.2 we would be able to give a formula for

$$
\sum_{a+p b=n} \sigma(a) \sigma(b) .
$$

\subsection{Further Discussions}

Let $f(z)$ be a modular form in $M_{k}\left(\Gamma_{0}(N), \chi\right)$. It is well known that we can write

$$
f(z)=E_{f}(z)+C_{f}(z)
$$

where $E_{f}(z) \in E_{k}\left(\Gamma_{0}(N), \chi\right)$ and $C_{f}(z) \in S_{k}\left(\Gamma_{0}(N), \chi\right)$, we refer to them as Eisenstein part and cusp part of the modular form $f(z)$, respectively.

In Subsections 12.5.1 12.5.3, we have further discussions on the interplay among eta quotients, Eisenstein series, newforms and elliptic curves. 


\subsubsection{A Set of Criteria for an Eta Quotient to be in $E_{k}\left(\Gamma_{0}(N), \chi\right)$}

In Chapter 11, we found Fourier coefficients of eta quotients in various modular form spaces. In Figure 11.4.1, we gave the number of eta quotients in $E_{2}\left(\Gamma_{0}(N)\right)$ for $N \leq 100$. There are many similarities between these eta quotients. Their exponents seem to obey certain rules. In the future we want to understand these rules.

Question: In (12.5.1), let $f(z)$ be an eta quotient given by

$$
f(z)=\prod_{1 \leq \delta \mid N} \eta^{r_{\delta}}(\delta z)
$$

Under what conditions is the cusp part of $f(z)$ equal to $0 ?\left(\right.$ or $\left.C_{f}(z)=0 ?\right)$

\subsubsection{On Weight 2 Newforms and Elliptic Curves}

In [49], weight 2 newforms were given as single eta quotients. In [7], Section 8.2 and Appendix B, we give many other weight 2 newforms as linear combinations of Eisenstein series and eta quotients. Recall that $S_{k}^{n e w}\left(\Gamma_{0}(N), \chi\right)$ is the subspace of $S_{k}\left(\Gamma_{0}(N), \chi\right)$ such that, see [70, p. 162]

$$
S_{k}\left(\Gamma_{0}(N), \chi\right)=S_{k}^{\text {old }}\left(\Gamma_{0}(N), \chi\right) \oplus S_{k}^{\text {new }}\left(\Gamma_{0}(N), \chi\right)
$$


Then by 1.0 .1 we have

$$
M_{k}\left(\Gamma_{0}(N), \chi\right)=E_{k}\left(\Gamma_{0}(N), \chi\right) \oplus S_{k}^{\text {old }}\left(\Gamma_{0}(N), \chi\right) \oplus S_{k}^{\text {new }}\left(\Gamma_{0}(N), \chi\right)
$$

That is, if $f(z) \in M_{k}\left(\Gamma_{0}(N), \chi\right)$ then we have

$$
\begin{aligned}
& f(z)=E_{f}(z)+S_{f}^{\text {old }}(z)+S_{f}^{\text {new }}(z), \\
& S_{f}^{\text {new }}(z)=f(z)-S_{f}^{\text {old }}(z)-E_{f}(z) .
\end{aligned}
$$

We obtained newforms as linear combinations of Eisenstein series and eta quotients in Chapter 8 and [7] using a computer search algorithm to find $f(z)-S_{f}^{\text {old }}(z)$ in terms of eta quotients and $E_{f}(z)$ in terms of Eisenstein series. In a sense the basic idea is to 'shave' eta quotients using Eisenstein series to obtain the 'newform component' of it. In future we wish to understand which eta quotients are 'shaveable'.

Question: Let $f(z) \in M_{k}\left(\Gamma_{0}(N), \chi\right)$ be a linear combination of eta quotients. Under which conditions there exists a modular form $E_{f}(z) \in E_{k}\left(\Gamma_{0}(N), \chi\right)$ such that $f(z)-E_{f}(z)$ is a newform.

Tackling this question will require similar calculations to the ones we are planning to do for the question raised in 12.5.1. Once we answer this question we can apply it to elliptic curves using the modularity theorem (Theorem 8.2.1) and we might be able to generalize Theorem 8.2.3 for a family of elliptic curves. 


\subsubsection{On Larger Weight Newforms}

In [60], Ponomarev uses theta series to represent newforms in squarefree levels. We hope to express all newforms in level 12 and even weight in terms of eta quotients. Let $f(z) \in S_{2 k}\left(\Gamma_{0}(12)\right)$ be a newform. Then by Theorem 5.1 .3 , we have

$$
f(z)=\sum_{1 \leq j \leq 4 k-5} c_{j} C_{j, 2 k}
$$

for some $c_{j} \in \mathbb{C}(1 \leq j \leq 4 k-5)$.

On the other hand, we can obtain first few Fourier coefficients of all newforms in $S_{2 k}\left(\Gamma_{0}(12)\right)$ for any $k \geq 1$ using modular symbols, see [70, Section 8.10.1], [27, Chapter 2]. Then, we can compute $c_{j} \in \mathbb{C}(1 \leq j \leq$ $4 k-5)$ by comparing the first few Fourier coefficients of both sides of equation 12.5.3. So for fixed $k \geq 1$, we can determine $c_{j} \in \mathbb{C}(1 \leq j \leq 4 k-5)$.

Our curiosity is towards finding a more general statement.

Question: Can we give $c_{j} \in \mathbb{C}(1 \leq j \leq 4 k-5)$ for all $k \geq 1$ in a closed form?

Similar questions hold for newforms in $S_{2 k}\left(\Gamma_{0}(20)\right)$ and $S_{2 k}\left(\Gamma_{0}(28)\right)$, whose bases are provided in Appendix A. 


\section{Bibliography}

[1] S. Ahlgren, Multiplicative relations in powers of Euler's product, J. Number Theory 89 (2001), no. 2, 222-233.

[2] S. Ahlgren, J. Rouse, Congruences for newforms and the index of the Hecke algebra, Proc. Amer. Math. Soc. 139 (2011), 1247-1261.

[3] A. Alaca, On the number of representations of a positive integer by certain quadratic forms in twelve variables, J. Comb. Number Theory 3 (2011), 167-177.

[4] A. Alaca, S.. Alaca and Z. S. Aygin, Fourier coefficients of a class of eta quotients of weight 2, Int. J. Number Theory 11 (2015), 2381-2392.

[5] A. Alaca, Ş. Alaca and Z. S. Aygin, Theta products and eta quotients of level 24 and weight 2, Accepted for publication, Functiones et Approximatio (2016). 
[6] A. Alaca, Ş. Alaca and Z. S. Aygin, A family of eta quotients and an extension of Ramanujan-Mordell theorem, Submitted for publication, (2016).

[7] A. Alaca, S.. Alaca and Z. S. Aygin, Eta quotients, Eisenstein series and elliptic curves, Submitted for publication, (2016).

[8] A. Alaca, Ş. Alaca and Z. S. Aygin, Fourier coefficients of eta quotients of weight 2 and levels 8, 9, 10, Submitted for publication, (2016).

[9] A. Alaca, Ş. Alaca and Z. S. Aygin, Fourier coefficients of two classes of eta quotients of weight 1, Submitted for publication, (2016).

[10] A. Alaca, Ş. Alaca, Z. S. Aygin and K. S. Williams, Infinite products with coefficients which vanish on certain arithmetic progressions, Int. J. Number Theory, accepted for publication.

[11] A. Alaca, Ş. Alaca, K. S. Williams, Some infinite products of Ramanujan type, Canad. Math. Bull. Vol. 52 (4), (2009), 481-492.

[12] A. Alaca, Ş. Alaca, K. S. Williams, Sums of $4 k$ squares: a polynomial approach, J. Comb. and Number Theory 1, (2009), no 2, 133-152.

[13] Ş. Alaca, Y. Kesiciog̃lu, Representations by certain octonary quadratic forms whose coefficients are 1, 2, 3 and 6, Int. J. Number Theory 10 (2014), 133-150. 
[14] Ş. Alaca, K. S. Williams, The number of representations of a positive integer by certain octonary quadratic forms, Funct. Approx. Comment. Math. 43 (2010), 45-54.

[15] T. M. Apostol, Modular Functions and Dirichlet Series in Number Theory, Springer-Verlag, New York, 1990.

[16] A. Berkovich, F. Patane, Binary quadratic forms and the Fourier coefficients of certain weight 1 eta-quotients, J. Number Theory 135 (2014), 185-220.

[17] B. C. Berndt, Number Theory in the Spirit of Ramanujan, SpringerVerlag, 1991.

[18] C. Breuil, B. Conrad, F. Diamond and R. Taylor, On the modularity of elliptic curves over $\mathbb{Q}$ : wild 3-adic exercises, J. Amer. Math. Soc. 14 (2001), 843-939.

[19] H. H. Chan, K. S. Chua, Representations of integers as sums of 32 squares, Ramanujan J., 7 (2003), no. 1-3, 79-89.

[20] H. H. Chan, S. Cooper, Powers of theta functions, Pacific J. Math. 235 (2008), no. 1, 1-14.

[21] R. Chapman, Coefficients of products of powers of eta functions, Ramanujan J. 5 (2001), 271-279. 
[22] H. Cohen, Sommes de carres, fonctions L et formes modulaires, C. R. Acad. Sci. Paris Sr. A-B 277 (1973), A827-A830.

[23] H. Cohen, J. Oesterle, Dimensions des espaces de formes modulaires, Proc. Second Internat. Conf., Univ. Bonn, Bonn, (1976), 69-78.

[24] S. Cooper, On sums of an even number of squares, and an even number of triangular numbers: an elementary approach based on Ramanujan's ${ }_{1} \psi_{1}$ summation formula, Contemp. Math., 291, Amer. Math. Soc., Providence, RI, (2001).

[25] S. Cooper, S. Gun, M. Hirschhorn, B. Ramakrishnan, Relations among Fourier coefficients of certain eta products, Integers 5, (2005), no. 1, A16, 1-6.

[26] S. Cooper, M. D. Hirschhorn, R. Lewis, Powers of Euler's Product and Related Identities, Ramanujan J. 4 (2000), no. 2, 137-155.

[27] J. E. Cremona, Algorithms for modular elliptic curves, Cambridge University Press, Cambridge, 1992.

[28] F. Diamond, J. Shurman, A first course in modular forms, Graduate Texts in Mathematics, 228. Springer-Verlag, New York, 2005.

[29] J. W. L. Glaisher, On the square of the series in which the coefficients are the sums of the divisors of the exponents, Mess. Math. 14 (1885), 156-163. 
[30] B. Gordon and D. Sinor, Multiplicative properties of $\eta$-products, Lecture Notes in Math., vol.1395, Springer, New York (1989), 173-200.

[31] E. Grosswald, Representations of integers as sums of squares, SpringerVerlag, New York, (1985).

[32] S. Gun, M. R. Murty, Divisors of Fourier coefficients of modular forms, New York J. Math., 20 (2014), 229-239.

[33] A. Hamieh, Ternary quadratic forms and half-integral weight modular forms, LMS J. Comput. Math., 15 (2012), 418-435.

[34] H. Iwaniec, Topics in Classical Automorphic Forms, Graduate Studies in Mathematics, 17. American Mathematical Society, Providence, RI, 1997.

[35] L. J. P. Kilford, Generating spaces of modular forms with $\eta$-quotients, J. Algebra Number Theory Appl. 8, (2007), no. 2, 213-226.

[36] L. J. P. Kilford, Modular forms, a classical and computational introduction, Imperial College Press, London, 2008.

[37] H. Kim, Eisenstein series on quaternion half-space, Manuscripta Math. 76 (1992), 85-104.

[38] M. I. Knopp, Modular functions in analytic number theory, Markham Publishing Co., Chicago, Ill. (1970).

[39] N. Koblitz, Introduction to elliptic curves and modular forms, Graduate Texts in Mathematics, 97. Springer-Verlag, New York, (1984). 
[40] G. Köhler, Eta products and theta series identities, Springer Monographs in Mathematics, Springer, Heidelberg, 2011.

[41] G. Köhler, Some eta-identities arising from theta series, Math. Scand. 66 (1990), no. 1, 147-154.

[42] W. Kohnen, Y. Martin, Products of two Eisenstein series and spaces of cusp forms of prime level, J. Ramanujan Math. Soc. 23 (2008), no. 4, 337-356.

[43] B. Köklüce, The representation numbers of three octonary quadratic forms, Int. J. Number Theory 9 (2013), 505-516.

[44] S. Lang, Introduction to modular forms, Springer-Verlag, Berlin, 1995.

[45] M. Lemire, Extensions of the Ramanujan-Mordell formula, $\mathrm{PhD}$ thesis, 2010, Carleton University.

[46] G. Ligozat, Courbes modulaires de genre 1, Bull. Soc. Math. France 43 (1975), 5-80.

[47] J. Lovejoy, Ramanujan-type congruences for three colored Frobenius partitions, J. Number Theory 85 (2000), no. 2, 283-290.

[48] Y. Martin, Multiplicative $\eta$-quotients, Trans. Amer. Math. Soc. 348 (1996), no. $12,4825-4856$.

[49] Y. Martin and K. Ono, Eta-quotients and Elliptic Curves, Proc. Amer. Math. Soc. 125 (1997), no. 11, 3169-3176. 
[50] S. C. Milne, Infinite families of exact sums of squares formulas, Jacobi elliptic functions, continued fractions, and Schur functions, The Ramanujan J., 6 (2002), no. 1, 7-149.

[51] T. Miyake, Modular Forms, Springer-Verlag, Berlin (1989), translated from the Japanese by Yoshitaka Maeda.

[52] H. L. Montgomery, R. C. Vaughan, Multiplicative Number Theory: I. Classical Theory, Cambridge University Press, Cambridge, (2007).

[53] L. J. Mordell, On the representations of numbers as a sum of 2r squares, Quart. J. Pure Appl. Math. 48 (1917), 93-104.

[54] M. Newman, An identity for the coefficients of certain modular forms, J. London Math. Soc. 30 (1955), 488-493.

[55] M. Newman, Construction and application of a certain class of modular functions II, Proc. London Math. Soc. (3) 9 (1959), 373-387.

[56] A. Okamoto, On expressions of theta series by $\eta$-products, Tokyo J. Math. 34 (2011), 319-326.

[57] K. Ono, Representations of integers as sums of squares, J. Number Theory 95 (2002), no. 2, 253-258.

[58] K. Ono, The Web of Modularity: Arithmetic of the Coefficients of Modular Forms and q-Series, Am. Math. Soc., Providence, RI, 2004. 
[59] D. Pathakjee, Z. RosnBrick and E. Yoong, Elliptic curves, eta-quotients and hypergeometric functions, Involve 5 (2012), no. 1, 1-18.

[60] P. Ponomarev, Newforms of squarefree level and theta series, Math. Ann. 345 (2009), no. 1, 185-193.

[61] B. Ramakrishnan and B. Sahu, Evaluation of the convolution sums $\sum_{l+15 m=n} \sigma(l) \sigma(m)$ and $\sum_{3 l+5 m=n} \sigma(l) \sigma(m)$ and an application, Int. J. Number Theory 9 (2013), no. 3, 799-809.

[62] S. Ramanujan, On certain arithmetical functions Trans. Cambridge Philos. Soc. 22 (1916), no. 9, 159-184.

[63] S. Ramanujan, Some properties of $p(n)$, the number of partitions of $n$ Proc. Cambridge Phil. Soc. 19 (1919), 207-210.

[64] S. Ramanujan, The lost notebook and other unpublished papers. Narosa Publishing House, New Delhi, 1988.

[65] J. Rouse, J. J. Webb, On spaces of modular forms spanned by etaquotients, Adv. Math. 272 (2015), 200-224.

[66] M. Sadek, On elliptic curves whose conductor is a product of two prime powers, Math. Comp. 83, no. 285, (2013), 447-460.

[67] J.-P. Serre, Modular forms of weight one and Galois representations, Algebraic number fields: L-functions and Galois properties (Proc. Sympos., Univ. Durham, Durham, 1975), Academic Press, London, 1977, 193-268. 
[68] J. H. Silverman, The arithmetic of elliptic curves, second edition Graduate Texts in Mathematics, 106. Springer, Dordrecht, (2009).

[69] W. A. Stein et al., SAGE Mathematics Software (Version 5.11), The SAGE Development Team, http://www.sagemath.org (2015).

[70] W. A. Stein, Modular forms, a computational approach, Graduate Studies in Mathematics, 79. American Mathematical Society, Providence, RI, (2007).

[71] A. Wiles, Modular elliptic curves and Fermat's last theorem, Ann. of Math. (2) 141 (1995), no. 3, 443-551.

[72] K. S. Williams, Fourier series of a class of eta quotients, Int. J. Number Theory 8 (2012), no. 4, 993-1004.

[73] D. Zagier, From number theory to physics, Chapter 4: Introduction to modular forms, Papers from the Meeting on Number Theory and Physics held in Les Houches, March 7-16, 1989. 


\section{Appendix A}

\section{Bases for $S_{2 k}\left(\Gamma_{0}(20)\right)$ and $S_{2 k}\left(\Gamma_{0}(28)\right)$}

Please note that we can give families of eta quotients which generate spaces with different levels as well. $C_{j, k}$ and $D_{j, k}$ are special because of their simplicity. For example we give families that generate $S_{2 k}\left(\Gamma_{0}(20)\right)$ and $S_{2 k}\left(\Gamma_{0}(28)\right)$ in the following theorem. Before we start let us fix some eta quotients by

$$
\begin{aligned}
& A(20, k ; z)=\left(\frac{\eta(z) \eta(4 z) \eta^{25 / 2}(10 z)}{\eta^{5 / 2}(2 z) \eta^{5}(5 z) \eta^{5}(20 z)}\right)^{k} \\
& B(20, j ; z)=\left(\frac{\eta^{3}(2 z) \eta^{5}(5 z) \eta^{10}(20 z)}{\eta(z) \eta^{2}(4 z) \eta^{15}(10 z)}\right)^{j} \\
& A(28, k ; z)=\left(\frac{\eta^{2 / 3}(z) \eta^{2 / 3}(4 z) \eta^{35 / 3}(14 z)}{\eta^{5 / 3}(2 z) \eta^{14 / 3}(7 z) \eta^{14 / 3}(28 z)}\right)^{k} \\
& B(28, j ; z)=\left(\frac{\eta^{1 / 2}(2 z) \eta^{7 / 6}(7 z) \eta^{7 / 3}(28 z)}{\eta^{1 / 6}(z) \eta^{1 / 3}(4 z) \eta^{7 / 2}(14 z)}\right)^{j}
\end{aligned}
$$


Theorem A.0.1. Let $k>2$ be an integer and $A(20, k ; z), B(20, j ; z), A(28, k ; z)$, $B(28, j ; z)$ be as in A.0.1 A.0.4. Then we have If $k \equiv 0(\bmod 4)$, then the union of the following sets of eta quotients form a basis for $S_{k}\left(\Gamma_{0}(20)\right)$ :

$$
\begin{aligned}
& \left\{A(20, k ; z) B(20, j ; z)\left(\frac{\eta^{8}(2 z) \eta^{15}(5 z) \eta^{13}(20 z)}{\eta^{3}(z) \eta(4 z) \eta^{32}(10 z)}\right)\right\}_{0 \leq j \leq k-3}, \\
& \left\{A(20, k ; z) B(20, j ; z)\left(\frac{\eta^{14}(2 z) \eta^{17}(5 z) \eta^{17}(20 z)}{\eta^{5}(z) \eta^{5}(4 z) \eta^{38}(10 z)}\right)\right\}_{0 \leq j \leq k-3}, \\
& \left\{A(20, k ; z) B(20, j ; z)\left(\frac{\eta^{9}(2 z) \eta^{15}(5 z) \eta^{18}(20 z)}{\eta^{3}(z) \eta^{2}(4 z) \eta^{37}(10 z)}\right)\right\}_{0 \leq j \leq k-3} .
\end{aligned}
$$

If $k \equiv 2(\bmod 4)$, then the union of following sets of eta quotients form a basis for $S_{k}\left(\Gamma_{0}(20)\right)$ :

$$
\begin{aligned}
& \left\{A(20, k ; z) B(20, j ; z)\left(\frac{\eta^{7}(2 z) \eta^{10}(5 z) \eta^{10}(20 z)}{\eta^{2}(z) \eta^{2}(4 z) \eta^{23}(10 z)}\right)\right\}_{0 \leq j \leq k-2}, \\
& \left\{A(20, k ; z) B(20, j ; z)\left(\frac{\eta^{13}(2 z) \eta^{15}(5 z) \eta^{17}(20 z)}{\eta^{3}(z) \eta^{5}(4 z) \eta^{37}(10 z)}\right)\right\}_{0 \leq j \leq k-3}, \\
& \left\{A(20, k ; z) B(20, j ; z)\left(\frac{\eta^{14}(2 z) \eta^{17}(5 z) \eta^{20}(20 z)}{\eta^{5}(z) \eta^{4}(4 z) \eta^{42}(10 z)}\right)\right\}_{0 \leq j \leq k-4} .
\end{aligned}
$$

If $k \equiv 0(\bmod 6)$, then the union of following sets of eta quotients form a basis for $S_{k}\left(\Gamma_{0}(28)\right)$ : 


$$
\begin{aligned}
& \left\{A(28, k ; z) B(28,6 j+1 ; z)\left(\frac{\eta^{7 / 2}(2 z) \eta^{35 / 6}(7 z) \eta^{14 / 3}(28 z)}{\eta^{5 / 6}(z) \eta^{2 / 3}(4 z) \eta^{25 / 2}(14 z)}\right)\right\}_{0 \leq j \leq \frac{4 k-6}{6}}, \\
& \left\{A(28, k ; z) B(28,6 j+2 ; z)\left(\frac{\eta^{4}(2 z) \eta^{41 / 3}(7 z) \eta^{31 / 3}(28 z)}{\eta^{1 / 3}(z) \eta^{1 / 3}(4 z) \eta^{28}(14 z)}\right)\right\}_{0 \leq j \leq \frac{4 k-12}{6}}, \\
& \left\{A(28, k ; z) B(28,6 j+3 ; z)\left(\frac{\eta^{9 / 2}(2 z) \eta^{21 / 2}(7 z) \eta^{7}(28 z)}{\eta^{3 / 2}(z) \eta^{1}(4 z) \eta^{39 / 2}(14 z)}\right)\right\}_{0 \leq j \leq \frac{4 k-12}{6}}, \\
& \left\{A(28, k ; z) B(28,6 j+4 ; z)\left(\frac{\eta^{3}(2 z) \eta^{7 / 3}(4 z) \eta^{28 / 3}(7 z) \eta^{23 / 3}(28 z)}{\eta^{4 / 3}(z) \eta^{21}(14 z)}\right)\right\}_{0 \leq j \leq \frac{4 k-12}{6}}, \\
& \left\{A(28, k ; z) B(28,6 j+5 ; z)\left(\frac{\eta^{15 / 2}(2 z) \eta^{85 / 6}(7 z) \eta^{31 / 3}(28 z)}{\eta^{19 / 6}(z) \eta^{1 / 3}(4 z) \eta^{57 / 2}(14 z)}\right)\right\}_{0 \leq j \leq \frac{4 k-18}{6}}, \\
& \left\{A(28, k ; z) B(28,6 j+6 ; z)\left(\frac{\eta(z) \eta^{3}(2 z) \eta^{9}(7 z) \eta^{8}(28 z)}{\eta^{21}(14 z)}\right)\right\}_{0 \geq j \geq \frac{4 k-12}{6}},
\end{aligned}
$$

If $k \equiv 2(\bmod 6)$, then the union of following sets of eta quotients form a basis for $S_{k}\left(\Gamma_{0}(28)\right)$ :

$$
\begin{aligned}
& \left\{A(28, k ; z) B(28,6 j+1 ; z)\left(\frac{\eta^{41 / 6}(2 z) \eta^{43 / 6}(7 z) \eta^{6}(28 z)}{\eta^{13 / 6}(z) \eta^{2}(4 z) \eta^{95 / 6}(14 z)}\right)\right\}_{0 \leq j \leq \frac{4 k-8}{6}}, \\
& \left\{A(28, k ; z) B(28,6 j+2 ; z)\left(\frac{\eta^{10 / 3}(2 z) \eta^{1 / 3}(4 z) \eta^{7}(7 z) \eta^{17 / 3}(28 z)}{\eta(z) \eta^{46 / 3}(14 z)}\right)\right\}_{0 \leq j \leq \frac{4 k-8}{6}}, \\
& \left\{A(28, k ; z) B(28,6 j+3 ; z)\left(\frac{\eta^{47 / 6}(2 z) \eta^{71 / 6}(7 z) \eta^{25 / 3}(28 z)}{\eta^{17 / 6}(z) \eta^{7 / 3}(4 z) \eta^{137 / 6}(14 z)}\right)\right\}_{0 \leq j \leq \frac{4 k-14}{6}-2,}, \\
& \left\{A(28, k ; z) B(28,6 j+4 ; z)\left(\frac{\eta^{13 / 3}(2 z) \eta^{35 / 3}(7 z) \eta^{8}(28 z)}{\eta^{5 / 3}(z) \eta^{67 / 3}(14 z)}\right)\right\}_{0 \leq j \leq \frac{4 k-14}{6}}, \\
& \left\{A(28, k ; z) B(28,6 j+5 ; z)\left(\frac{\eta^{59 / 6}(2 z) \eta^{21 / 2}(7 z) \eta^{29 / 3}(28 z)}{\eta^{3 / 2}(z) \eta^{11 / 3}(4 z) \eta^{149 / 6}(14 z)}\right)\right\}_{0 \leq j \leq \frac{4 k-14}{6}} \\
& \left\{A(28, k ; z) B(28,6 j+6 ; z)\left(\frac{\eta^{19 / 3}(2 z) \eta^{31 / 3}(7 z) \eta^{28 / 3}(28 z)}{\eta^{1 / 3}(z) \eta^{4 / 3}(4 z) \eta^{73 / 3}(14 z)}\right)\right\}_{0 \leq j \leq \frac{4 k-20}{6}}
\end{aligned}
$$


If $k \equiv 4(\bmod 6)$, then the union of following sets of eta quotients form a basis for $S_{k}\left(\Gamma_{0}(28)\right)$ :

$$
\begin{aligned}
& \left\{A(28, k ; z) B(28,6 j+1 ; z)\left(\frac{\eta^{61 / 6}(2 z) \eta^{17 / 2}(7 z) \eta^{22 / 3}(28 z)}{\eta^{7 / 2}(z) \eta^{10 / 3}(4 z) \eta^{15 / 6}(14 z)}\right)\right\}_{0 \leq j \leq \frac{4 k-10}{6}}, \\
& \left\{A(28, k ; z) B(28,6 j+2 ; z)\left(\frac{\eta^{20 / 3}(2 z) \eta^{25 / 3}(7 z) \eta^{7}(28 z)}{\eta^{7 / 3}(z) \eta(4 z) \eta^{56 / 3}(14 z)}\right)\right\}_{0 \leq j \leq \frac{4 k-10}{6}}, \\
& \left\{A(28, k ; z) B(28,6 j+3 ; z)\left(\frac{\eta^{19 / 6}(2 z) \eta^{4 / 3}(4 z) \eta^{49 / 6}(7 z) \eta^{20 / 3}(28 z)}{\eta^{7 / 6}(z) \eta^{109 / 6}(14 z)}\right)\right\}_{0 \leq j \leq \frac{4 k-10}{6}}, \\
& \left\{A(28, k ; z) B(28,6 j+4 ; z)\left(\frac{\eta^{23 / 3}(2 z) \eta^{13}(7 z) \eta^{28 / 3}(28 z)}{\eta^{3}(z) \eta^{4 / 3}(4 z) \eta^{77 / 3}(14 z)}\right)\right\}_{0 \leq j \leq \frac{4 k-16}{6}}, \\
& \left\{A(28, k ; z) B(28,6 j+5 ; z)\left(\frac{\eta^{7 / 6}(z) \eta^{19 / 6}(2 z) \eta^{47 / 6}(7 z) \eta^{7}(28 z)}{\eta(4 z) \eta^{109 / 6}(14 z)}\right)\right\}_{0 \leq j \leq \frac{4 k-16}{6}}, \\
& \left\{A(28, k ; z) B(28,6 j+6 ; z)\left(\frac{\eta^{29 / 3}(2 z) \eta^{35 / 3}(7 z) \eta^{32 / 3}(28 z)}{\eta^{5 / 3}(z) \eta^{8 / 3}(4 z) \eta^{83 / 3}(14 z)}\right)\right\}_{0 \leq j \leq \frac{4 k-16}{6}} .
\end{aligned}
$$




\section{Appendix B}

\section{Newforms in $M_{2}\left(\Gamma_{0}(N)\right)$ for various $N<100$}

Table 3.1: Weight 2 newforms

\begin{tabular}{lll}
\hline \hline Level & Eisenstein series and eta quotients \\
\hline \hline 17 & $F_{17}(z)=$ & $\eta_{68}[1,-1,2,-1,5,-2](z)-\eta_{68}[-1,5,-2,1,-1,2](z)$ \\
\hline 19 & $F_{19}(z)=$ & $6 \eta_{76}[0,-2,4,0,-2,4](z)+3 \eta_{76}[-2,4,0,-2,4,0](z)$ \\
& & $+\frac{3}{8} \eta_{76}[-4,10,-4,-4,10,-4](z)-\frac{1}{2} L_{19}(z)$ \\
\hline 21 & $F_{21}(z)=\quad-2 \eta_{42}[-2,1,6,-3,-2,1,6,-3](z)$ \\
& & $+4 \eta_{42}[-1,2,2,-1,-1,2,2,-1](z)$ \\
& & $-\frac{1}{3} L_{3}(z)+\frac{4}{3} L_{6}(z)-L_{7}(z)-\frac{1}{3} L_{21}(z)+\frac{4}{3} L_{42}(z)$ \\
\hline $26 A$ & $F_{26 A}(z)=$ & $-3 \eta_{52}[-1,1,2,-3,7,-2](z)-3 \eta_{52}[-3,7,-2,-1,1,2](z)$ \\
& & $+\frac{1}{2} L_{2}(z)+L_{13}(z)-\frac{1}{2} L_{26}(z)$ \\
\hline $26 B$ & $F_{26 B}(z)=$ & $7 \eta_{52}[0,-2,4,0,-2,4](z)+\frac{7}{16} \eta_{52}[-4,10,-4,-4,10,-4](z)$ \\
& & $-\frac{3}{4} L_{2}(z)+\frac{3}{4} L_{13}(z)-\frac{3}{4} L_{26}(z)$ \\
\hline
\end{tabular}




\begin{tabular}{|c|c|c|}
\hline Level & & Eisenstein series and eta quotients \\
\hline 33 & $F_{33}(z)=$ & $\begin{array}{l}-6 \eta_{33}[-1,3,-1,3](z)-\frac{12}{5} \eta_{33}[0,2,0,2](z)+\frac{2}{5} \eta_{33}[2,0,2,0](z) \\
+\frac{1}{5} L_{3}(z)+\frac{3}{5} L_{11}(z)-\frac{1}{5} L_{33}(z)\end{array}$ \\
\hline 35 & $F_{35}(z)=$ & $\eta_{35}[0,2,2,0](z)+\eta_{35}[2,0,0,2](z)$ \\
\hline $38 A$ & $F_{38 A}(z)=$ & $\begin{array}{l}-3 \eta_{76}[0,-2,4,0,-2,4](z)-3 \eta_{76}[-2,4,0,-2,4,0](z) \\
-\frac{3}{16} \eta_{76}[-4,10,-4,-4,10,-4](z)-\frac{3}{8} \eta_{76}[4,-2,0,4,-2,0](z) \\
-\frac{1}{4} L_{2}(z)+\frac{1}{4} L_{19}(z)+\frac{1}{4} L_{38}(z)\end{array}$ \\
\hline $38 B$ & $F_{38 B}(z)=$ & $\eta_{38}[-1,3,3,-1](z)+\eta_{38}[3,-1,-1,3](z)$ \\
\hline 40 & $F_{40}(z)=$ & $\begin{array}{l}-4 \eta_{40}[2,-1,-1,2,2,-1,-1,2](z)+\frac{3}{2} L_{2}(z)+\frac{3}{2} L_{4}(z) \\
+L_{5}(z)-L_{8}(z)-\frac{3}{2} L_{10}(z)-\frac{3}{2} L_{20}(z)+L_{40}(z)\end{array}$ \\
\hline 42 & $F_{42}(z)=$ & $\begin{array}{l}-12 \eta_{42}[0,0,-2,4,0,0,-2,4](z)-8 \eta_{42}[0,-1,0,3,-1,0,3,0](z) \\
+L_{2}(z)-L_{3}(z)+\frac{1}{3} L_{6}(z)-L_{7}(z)+L_{14}(z)+\frac{5}{3} L_{21}(z)-L_{42}(z)\end{array}$ \\
\hline 45 & $F_{45}(z)=$ & $\begin{array}{l}2 \eta_{45}[-1,2,2,-1,0,2](z)-2 \eta_{45}[-2,6,1,-2,0,1](z) \\
+\frac{1}{5} L_{5}(z)+L_{9}(z)-\frac{1}{5} L_{45}(z)\end{array}$ \\
\hline 46 & $F_{46}(z)=$ & $\begin{array}{l}\frac{16}{5} \eta_{46}[-1,3,-1,3](z)+2 \eta_{46}[0,2,0,2](z)+\frac{2}{5} \eta_{46}[1,1,1,1](z) \\
-\frac{11}{40} \eta_{46}[4,-2,4,-2](z)-\frac{1}{5} L_{2}(z)-\frac{1}{10} L_{23}(z)+\frac{1}{5} L_{46}(z)\end{array}$ \\
\hline 52 & $F_{52}(z)=$ & $\begin{array}{l}-2 \eta_{52}[0,-2,4,0,-2,4](z)+\frac{1}{8} \eta_{52}[-4,10,-4,-4,10,-4](z) \\
-\frac{1}{8} \eta_{52}[4,-2,0,4,-2,0](z)\end{array}$ \\
\hline $54 A$ & $F_{54 A}(z)=$ & $\begin{array}{l}-\frac{3}{4} \eta_{54}[-2,1,4,-1,4,-1,-2,1](z) \\
+\frac{3}{4} \eta_{54}[2,-1,0,1,0,1,2,-1](z) \\
+\frac{1}{2} L_{2}(z)+\frac{1}{3} L_{3}(z)-\frac{1}{6} L_{6}(z) \\
-\frac{1}{3} L_{9}(z)+\frac{1}{6} L_{18}(z)+L_{27}(z)-\frac{1}{2} L_{54}(z)\end{array}$ \\
\hline
\end{tabular}




\begin{tabular}{lll}
\hline \hline Level & Eisenstein series and eta quotients \\
\hline \hline $63 \quad F_{63}(z)=\quad$ & $\frac{4}{3} \eta_{63}[0,-1,3,3,-1,0](z)+\frac{4}{3} \eta_{63}[3,-1,0,0,-1,3](z)$ \\
& $-\frac{1}{3} E_{2, \chi-3, \chi-3}(z)-\frac{7}{3} E_{2, \chi_{-3}, \chi_{-3}}(7 z)$ \\
\hline $70 \quad F_{70}(z)=\quad$ & $-4 \eta_{70}[0,2,-1,1,1,-1,2,0](z)-4 \eta_{70}[2,0,1,-1,-1,1,0,2](z)$ \\
& $+L_{2}(z)+3 L_{5}(z)+3 L_{7}(z)-3 L_{10}(z)$ \\
& $-3 L_{21}(z)-L_{35}(z)+L_{70}(z)$ \\
\hline $75 A \quad F_{75 A}(z)=$ & $-20 \eta_{75}[0,0,4,-2,-2,4](z)+3 \eta_{75}[0,1,1,1,1,0](z)$ \\
& $+3 \eta_{75}[1,0,1,1,0,1](z)-4 \eta_{75}[4,-2,-2,4,0,0](z)$ \\
& $+7 L_{3}(z)-8 L_{5}(z)+8 L_{15}(z)-7 L_{25}(z)+L_{75}(z)$ \\
\hline $75 B \quad F_{75 B}(z)=$ & $2 \eta_{75}[-1,2,1,1,2,-1](z)-2 \eta_{75}[2,-1,1,1,-1,2](z)$ \\
& $-L_{3}(z)-\frac{2}{5} L_{5}(z)+\frac{2}{5} L_{15}(z)+L_{25}(z)-L_{75}(z)$ \\
\hline $75 C \quad F_{75 C}(z)=$ & $2 \eta_{75}[-1,2,1,1,2,-1](z)-3 \eta_{75}[0,1,1,1,1,0](z)$ \\
& $+3 \eta_{75}[1,0,1,1,0,1](z)-2 \eta_{75}[2,-1,1,1,-1,2](z)$ \\
& $-L_{3}(z)-\frac{2}{5} L_{5}(z)+\frac{2}{5} L_{15}(z)+L_{25}(z)-L_{75}(z)$ \\
\hline & $\frac{42}{5} \eta_{98}[0,0,-3,5,5,-3](z)-3 \eta_{98}[-2,4,-2,4,0,0](z)$ \\
& $+4 \eta_{98}[0,1,1,1,1,0](z)+4 \eta_{98}[1,0,1,1,0,1](z)$ \\
& $+\frac{1}{2} L_{2}(z)+\frac{18}{5} L_{7}(z)-\frac{3}{2} L_{14}(z)+L_{49}(z)-\frac{13}{5} L_{98}(z)$ \\
\hline $\left.98 \quad F_{98}(z)=-1,4,-1,-1,4,-1\right](z)-2 \eta_{99}[0,2,0,0,2,0](z)$ \\
\end{tabular}




\section{Appendix $\mathrm{C}$}

\section{Bases for $M_{2}\left(\Gamma_{0}(N)\right)$ for various $N<100$}

In the Table C.0.1, we give bases for $M_{2}\left(\Gamma_{0}(N)\right)$ for all $N \leq 100$ whose cusp form subspace can be generated by eta quotients whose orders at infinity are different.

Table C.0.1: Bases for $M_{2}\left(\Gamma_{0}(N)\right)$

\begin{tabular}{|l|l|l|}
\hline \hline Space & Eisenstein Basis & Cusp Basis \\
\hline$M_{2}\left(\Gamma_{0}(11)\right)$ & $L_{11}(z)$ & $\eta_{11}[2,2](z)$ \\
\hline$M_{2}\left(\Gamma_{0}(12)\right)$ & $\left\{L_{t}(z)|1<t| 12\right\}$ & - \\
\hline$M_{2}\left(\Gamma_{0}(14)\right)$ & $\left\{L_{t}(z)|1<t| 14\right\}$ & $\eta_{14}[1,1,1,1](z)$ \\
\hline$M_{2}\left(\Gamma_{0}(15)\right)$ & $\left\{L_{t}(z)|1<t| 15\right\}$ & $\eta_{15}[1,1,1,1](z)$ \\
\hline$M_{2}\left(\Gamma_{0}(16)\right)$ & $\left\{L_{t}(z)|1<t| 16\right\}$ & - \\
& $E_{2, \chi_{-4}, \chi_{-4}}(z)$ & \\
\hline$M_{2}\left(\Gamma_{0}(20)\right)$ & $\left\{L_{t}(z)|1<t| 20\right\}$ & $\eta_{20}[0,2,0,0,2,0](z)$ \\
\hline
\end{tabular}




\begin{tabular}{|c|c|c|}
\hline Space & Eisenstein Basis & Cusp Basis \\
\hline$M_{2}\left(\Gamma_{0}(24)\right)$ & $\left\{L_{t}(z)|1<t| 24\right\}$ & $\eta_{24}[0,1,0,1,1,0,1,0](z)$ \\
\hline$M_{2}\left(\Gamma_{0}(27)\right)$ & $\begin{array}{l}\left\{L_{t}(z)|1<t| 27\right\} \\
E_{2, \chi_{-3}, \chi_{-3}}(z) \\
E_{2, \chi_{-3}, \chi_{-3}}(3 z)\end{array}$ & $\eta_{27}[0,2,2,0](z)$ \\
\hline$M_{2}\left(\Gamma_{0}(28)\right)$ & $\left\{L_{t}(z)|1<t| 28\right\}$ & $\begin{array}{l}\eta_{28}[1,1,0,1,1,0](z) \\
\eta_{28}[0,1,1,0,1,1](z)\end{array}$ \\
\hline$M_{2}\left(\Gamma_{0}(30)\right)$ & $\left\{L_{t}(z)|1<t| 30\right\}$ & $\begin{array}{l}\eta_{30}[1,-1,-1,-1,3,3,1,-1](z) \\
\eta_{30}[2,-1,-1,-1,2,2,2,-1](z) \\
\eta_{30}[3,-1,-1,-1,1,1,3,-1](z)\end{array}$ \\
\hline$M_{2}\left(\Gamma_{0}(32)\right)$ & $\begin{array}{l}\left\{L_{t}(z)|1<t| 32\right\} \\
E_{2, \chi_{-4}, \chi_{-4}}(z) \\
E_{2, \chi_{-4}, \chi_{-4}}(2 z)\end{array}$ & $\eta_{32}[0,0,2,2,0,0](z)$ \\
\hline$M_{2}\left(\Gamma_{0}(33)\right)$ & $\left\{L_{t}(z)|1<t| 33\right\}$ & $\begin{array}{l}\eta_{33}[2,0,2,0](z) \\
\eta_{33}[1,1,1,1](z) \\
\eta_{33}[0,2,0,2](z)\end{array}$ \\
\hline$M_{2}\left(\Gamma_{0}(35)\right)$ & $\left\{L_{t}(z)|1<t| 35\right\}$ & $\begin{array}{l}\eta_{35}[0,2,2,0](z) \\
\eta_{35}[1,1,1,1](z) \\
\eta_{35}[2,0,0,2](z)\end{array}$ \\
\hline$M_{2}\left(\Gamma_{0}(36)\right)$ & $\begin{array}{l}\left\{L_{t}(z)|1<t| 36\right\} \\
E_{2, \chi_{-3}, \chi_{-3}}(z) \\
E_{2, \chi_{-3}, \chi_{-3}}(2 z)\end{array}$ & $\eta_{36}[0,0,0,0,4,0,0,0,0](z)$ \\
\hline
\end{tabular}




\begin{tabular}{|c|c|c|}
\hline Space & Eisenstein Basis & Cusp Basis \\
\hline & $E_{2, \chi_{-3}, \chi_{-3}}(4 z)$ & \\
\hline$M_{2}\left(\Gamma_{0}(40)\right)$ & $\left\{L_{t}(z)|1<t| 40\right\}$ & $\begin{array}{l}\eta_{40}[0,1,-1,0,2,-1,5,-2](z) \\
\eta_{40}[1,0,0,-1,1,2,2,-1](z) \\
\eta_{40}[2,-1,1,-2,0,5,-1,0](z)\end{array}$ \\
\hline$M_{2}\left(\Gamma_{0}(46)\right)$ & $\left\{L_{t}(z)|1<t| 46\right\}$ & $\begin{array}{l}\eta_{46}[3,-1,3,-1](z) \\
\eta_{46}[2,0,2,0](z) \\
\eta_{46}[1,1,1,1](z) \\
\eta_{46}[0,2,0,2](z) \\
\eta_{46}[-1,3,-1,3](z)\end{array}$ \\
\hline$M_{2}\left(\Gamma_{0}(48)\right)$ & $\begin{array}{l}\left\{L_{t}(z)|1<t| 48\right\} \\
E_{2, \chi_{-4, \chi-4}}(z) \\
E_{2, \chi_{-4}, \chi_{-4}}(3 z)\end{array}$ & $\begin{array}{l}\eta_{48}[0,0,0,2,0,-2,0,2,4,-2](z) \\
\eta_{48}[1,-1,-1,2,2,-1,0,1,2,-1](z) \\
\eta_{48}[2,-2,-2,2,4,0,0,0,0,0](z)\end{array}$ \\
\hline$M_{2}\left(\Gamma_{0}(54)\right)$ & $\begin{array}{l}\left\{L_{t}(z)|1<t| 54\right\} \\
E_{2, \chi_{-3}, \chi_{-3}}(z) \\
E_{2, \chi_{-3}, \chi_{-3}}(2 z) \\
E_{2, \chi_{-3}, \chi_{-3}}(3 z) \\
E_{2, \chi_{-3}, \chi_{-3}}(6 z)\end{array}$ & $\begin{array}{l}\eta_{54}[0,0,1,0,0,2,-1,2](z) \\
\eta_{54}[0,0,3,-1,-1,3,0,0](z) \\
\eta_{54}[2,-1,0,1,1,0,-1,2](z) \\
\eta_{54}[2,-1,2,0,0,1,0,0](z)\end{array}$ \\
\hline$M_{2}\left(\Gamma_{0}(56)\right)$ & $\left\{L_{t}(z)|1<t| 56\right\}$ & $\begin{array}{l}\eta_{56}[0,0,1,0,1,0,1,1](z) \\
\eta_{56}[0,-1,3,0,0,3,-1,0](z) \\
\eta_{56}[0,-1,4,0,-1,-1,4,1](z) \\
\eta_{56}[-1,3,-1,-1,1,3,-1,1](z)\end{array}$ \\
\hline
\end{tabular}


174

\begin{tabular}{|c|c|c|}
\hline Space & Eisenstein Basis & Cusp Basis \\
\hline & & $\eta_{56}[-1,2,2,-1,-1,2,2,-1](z)$ \\
\hline$M_{2}\left(\Gamma_{0}(60)\right)$ & $\left\{L_{t}(z)|1<t| 60\right\}$ & $\begin{array}{l}\eta_{60}[0,1,0,-1,0,-1,1,3,0,-1,-1,3](z) \\
\eta_{60}[1,0,-1,-1,-1,1,1,2,1,2,0,-1](z) \\
\eta_{60}[2,-2,-2,1,-2,4,4,-1,2,-1,-2,1](z) \\
\eta_{60}[2,0,-1,-1,-1,0,0,2,2,2,0,-1](z) \\
\eta_{60}[3,-2,-2,1,-2,3,3,-1,3,-1,-2,1](z) \\
\eta_{60}[3,-1,-1,0,0,1,1,0,0,-1,-1,3](z) \\
\eta_{60}[3,-1,-1,0,3,1,-1,0,-1,0,1,0](z)\end{array}$ \\
\hline$M_{2}\left(\Gamma_{0}(64)\right)$ & $\begin{array}{l}\left\{L_{t}(z)|1<t| 64\right\} \\
E_{2, \chi_{-8}, \chi_{-8}}(z) \\
E_{2, \chi_{8}, \chi_{8}}(z) \\
E_{2, \chi_{-4}, \chi_{-4}}(z) \\
E_{2, \chi_{-4}, \chi_{-4}}(2 z) \\
E_{2, \chi_{-4}, \chi_{-4}}(4 z)\end{array}$ & $\begin{array}{l}\eta_{64}[0,0,0,3,0,-1,2](z) \\
\eta_{64}[0,0,2,2,0,0,0](z) \\
\eta_{64}[2,-1,0,3,0,0,0](z)\end{array}$ \\
\hline$M_{2}\left(\Gamma_{0}(72)\right)$ & $\begin{array}{l}\left\{L_{t}(z)|1<t| 72\right\} \\
E_{2, \chi_{-3}, \chi_{-3}}(z) \\
E_{2, \chi_{-3}, \chi_{-3}}(2 z) \\
E_{2, \chi_{-3}, \chi_{-3}}(4 z) \\
E_{2, \chi_{-3}, \chi_{-3}}(8 z)\end{array}$ & $\begin{array}{l}\eta_{72}[0,0,0,0,2,0,0,0,-2,0,4,0](z) \\
\eta_{72}[1,-1,-2,-1,4,1,1,4,-1,-2,-1,1](z) \\
\eta_{72}[1,0,0,-1,-1,0,-1,5,3,0,-2,0](z) \\
\eta_{72}[2,-1,0,0,0,0,-2,2,5,0,-2,0](z) \\
\eta_{72}[3,-2,-1,1,2,0,0,1,0,0,0,0](z)\end{array}$ \\
\hline$M_{2}\left(\Gamma_{0}(80)\right)$ & $\begin{array}{l}\left\{L_{t}(z)|1<t| 80\right\} \\
E_{2, \chi_{-4}, \chi_{-4}}(z)\end{array}$ & $\begin{array}{l}\eta_{80}[0,0,1,0,-1,0,2,1,-1,2](z) \\
\eta_{80}[0,0,-1,2,5,-1,-2,1,0,0](z)\end{array}$ \\
\hline
\end{tabular}




\begin{tabular}{|l|l|l|}
\hline \hline Space & Eisenstein Basis & Cusp Basis \\
\hline & $E_{2, \chi_{-4}, \chi_{-4}}(5 z)$ & $\eta_{80}[1,-1,0,-1,3,3,-1,0,-1,1](z)$ \\
& & $\eta_{80}[1,0,0,-1,2,2,-1,0,0,1](z)$ \\
& & $\eta_{80}[1,1,0,-1,0,1,0,2,0,0](z)$ \\
& & $\eta_{80}[2,-1,1,0,0,0,0,1,-1,2](z)$ \\
& & $\eta_{80}[2,-1,1,2,0,-1,0,1,0,0](z)$ \\
\hline
\end{tabular}

